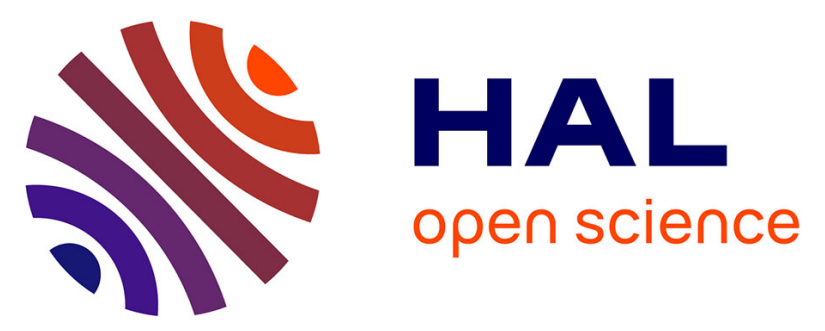

\title{
Les mutations d'un domaine de La Tène au haut Moyen Âge: Le Vernai à Saint-Romain-de-Jalionas (Isère)
} Robert Royet, Jean-François Berger, Colette Laroche, Elvyre Royet, Jacqueline Argant, Nicolas Bernigaud, Laurent Bouby, Thi-Mai-Anh Bui, Vianney Forest, José Antonio López Sáez

\section{To cite this version:}

Robert Royet, Jean-François Berger, Colette Laroche, Elvyre Royet, Jacqueline Argant, et al.. Les mutations d'un domaine de La Tène au haut Moyen Âge: Le Vernai à Saint-Romain-de-Jalionas (Isère). Gallia - Archéologie de la France antique, 2006, 63, pp.283-325. 10.3406/galia.2006.3299 . hal-01911084

\section{HAL Id: hal-01911084 \\ https://hal.science/hal-01911084}

Submitted on 8 Jan 2020

HAL is a multi-disciplinary open access archive for the deposit and dissemination of scientific research documents, whether they are published or not. The documents may come from teaching and research institutions in France or abroad, or from public or private research centers.
L'archive ouverte pluridisciplinaire HAL, est destinée au dépôt et à la diffusion de documents scientifiques de niveau recherche, publiés ou non, émanant des établissements d'enseignement et de recherche français ou étrangers, des laboratoires publics ou privés.

\section{(1) (1) $\$$}

Distributed under a Creative Commons Attribution - NonCommercial - NoDerivatives 44.0 


\title{
LES MUTATIONS D'UN DOMAINE DE LA TĖNE AU HAUT MOYEN ÂGE
}

\section{Le Vernai à Saint-Romain-de-Jalionas (Isère)}

\author{
Robert ROYET ${ }^{1}$, Jean-François BERGER ${ }^{2}$, Colette LAROCHE ${ }^{1}$, Elvyre ROYET ${ }^{3}$, \\ Jacqueline ARGANT ${ }^{4}$, Nicolas BERNiGAUd ${ }^{2}$, Laurent BOUBY ${ }^{2}$, Maï BUI THI ${ }^{2}$, \\ Vianney FOREST ${ }^{5}$, Antonio LOPEZ-SAEZ ${ }^{6}$
}

\begin{abstract}
Mots-clés. Villa, marais, réseaux hydrauliques, études environnementales.
Résumé. Une étude interdisciplinaire est actuellement conduite sur une villa et son terroir à Saint-Romain-de-Jalionas (Isère), aux confins septentrionaux de la Narbonnaise. À la fouille d'un établissement rural occupé sans interruption du III $s$. av. J.-C. à la fin du haut Moyen Âge, cette recherche associe l'exploration des formations superficielles de la plaine alluviale de la rivière bordant le site à celle d'un marais où sont conservés plusieurs réseaux hydrauliques antiques. Cet article présente les différentes phases de l'évolution de cet habitat, aux dimensions exceptionnelles, et les modalités de l'adaptation par ses occupants successifs d'un environnement considéré, a priori, comme défavorable.
\end{abstract}

Key-words. Villa, marsh, hydraulic system, environmental studies.

Abstract. A pluridisciplinary study is going on at Saint-Romain-de-Jalionas (Isère) on a villa and its territory on the northern fringes of Narbonensis. This research includes the excavation of a rural establishment occupied without interruption from the $3^{\text {rd }} c$. BC to the end of early Middle Age and the exploration of superficial formations of the alluvial valley of the river along the site and of a marsh where several Roman hydraulic systems are preserved. In this article, the different phases of occupation of this exceptionally vast settlement are being described altogether, with the modes of adaptation by its successive inhabitants of an environment considered as unfavourable.

Translation: Isabelle FAUDUET

Schlagwörter. Villa, Sumpfgebiet, Wasserleitungssystem, Umweltstudien.

Zusammenfassung. Derzeit wird eine interdisziplinäre Studie zu einer Villa bei Saint-Romain-de-Jalionas (Dép. Isère) und deren Territorium am Nordrand der Narbonnensis durchgeführt. Im Zusammenhang mit der Ausgrabung einer ländlichen Siedlung, die vom dritten vorchristlichen Jahrhundert bis zum Ausgang des frühen Mittelalters kontinuierlich belegt war, werden im Rahmen dieser Untersuchung die Geländeformen der Schwemmebene eines den Platz abgrenzenden Flußlaufs und eines Sumpfgebietes erforscht, in dem Teile mehrerer antiker Wasserleitungssysteme erhalten sind. Der Beitrag stellt die verschiedenen Entwicklungsstadien dieser ausnehmend

\footnotetext{
1. Service régional de l'archéologie en Rhône-Alpes, 6 quai Saint-Vincent, F-69283 Lyon Cedex 01. Courriels: robert.royet@culture.gouv.fr; colette.laroche@culture.gouv.fr

2. UMR 6130 du CNRS, CÉPAM, UNSA, 250 avenue Albert-Einstein, Sophia-Antipolis, F-06560 Valbonne. Courriels: berger@cepam.cnrs.fr; nicolas.bernigod@wanadoo.fr; bouby@cepam.cnrs.fr; buithi@cepam.cnrs.fr

3. La Rivoire, F-38460 Vénérieu.

4. UMR 6636 du CNRS, ESEP, Institut Dolomieu, 15 rue Maurice-Gignoux, F-38031 Grenoble Cedex. Courriel: j.argant@wanadoo.fr

5. Résidence Cap Ponant, 196 allée des Colverts, F-34280 La Grande Motte. Courriel: vianney.forest@inrap.fr

6. Laboratorio de arquéobotanica, Instituto de Historia, Consejo superior de investigaciones cientificas, Duque de Medinaceli 6, E-28014 Madrid. Courriel: alopez@ch.csic.es
} 
groß dimensionierten Siedlung vor und zeigt die Umstände, unter denen die aufeinanderfolgenden Nutzer sich einer Umwelt angepaßt haben, die dafür eigentlich als ungünstig gilt.

Übersetzung: Stefan WIRTH

À une trentaine de kilomètres à l'est de Lyon, sur la commune de Saint-Romain-de-Jalionas (Isère), se trouve une des plus importantes villa de la cité viennoise. Le terroir de la portion de la vallée du Rhône qu'elle contrôle apparaît pourtant en grande partie aride et peu fertile. L'actuelle clairière du Vernai où elle est installée est elle-même située en bordure d'un marais et subit de fréquentes inondations. Quelle que soit l'échelle d'analyse, l'environnement semble donc peu favorable à un établissement durable. Le Vernai a pourtant accueilli un habitat permanent entre La Tène et le début de l'époque moderne. Une ferme allobroge est à l'origine de la villa qui s'implante dès le $\mathrm{I}^{\mathrm{er}} \mathrm{s}$. av. J.-C. et évolue jusqu'à la fin de l'Antiquité. À cet établissement d'ampleur inhabituelle succède, au cours du haut Moyen Âge, une résidence qui, par certains aspects, prolonge la villa.

Ce gisement est actuellement l'objet d'un programme d'étude associant fouille de l'habitat et exploration de son environnement. Cette recherche vise à mieux comprendre les modalités de cette continuité exceptionnelle en croisant les données architecturales et économiques recueillies sur le site avec une étude naturaliste conduite au cœur de son terroir, notamment sur le marais du Grand-Plan et sur les berges de la rivière qui le bordent, le Girondan.

Cette étude présente les principales données archéologiques et paléoenvironnementales obtenues pour la période comprise entre La Tène finale et le haut Moyen Âge.

Une présentation chronologique intégrant les différentes contributions a été préférée à la juxtaposition d'interventions thématiques. La présentation de l'époque antique a été scindée en Haut- et Bas-Empire, division à l'intérieur de laquelle les évolutions sont présentées par secteur.

\section{PRÉSENTATION ET CONTEXTE}

\section{LE CONTEXTE GÉOLOGIQUE ET GÉOMORPHOLOGIQUE}

Saint-Romain-de-Jalionas est une commune du nord de l'Isère sise sur la rive gauche du Rhône. Le fleuve qui, en amont, était encadré par les massifs du Bugey et de l'Isle-Crémieu, voit ici sa vallée s'élargir en débouchant dans la vaste plaine fluvio-glaciaire du Velin (fig. 1). SaintRomain forme un rectangle d'environ 1000 ha divisible en deux ensembles pédologiques de superficies équivalentes. $\mathrm{Au}$ nord, la terrasse fluvio-glaciaire riche en galets est recouverte d'un sol rouge fersiallitique peu épais et très perméable. Seuls de petits affluents du Rhône (le Girondan, la Girine), provenant de résurgences situées au pied du massif calcaire de l'Isle-Crémieu rompent la monotonie de cette partie de la commune. Dans la moitié méridionale, la dernière glaciation a modelé une morphologie plus variée. Sur la terrasse graveleuse sont posées des moraines au pied desquelles s'étire un chapelet de dépressions humides. Ces reliefs consécutifs à la progression, puis à la fusion des langues glaciaires d'origine alpine, ont bloqué les écoulements superficiels suintant des piémonts du massif de l'IsleCrémieu. Un milieu palustre s'est ainsi développé dans ces cuvettes creusées par le glacier.

Le site du Vernai est dominé par la Butte-des-Vignes, une moraine en arc de cercle d'environ $1 \mathrm{~km}$ de diamètre qui surplombe la plaine environnante d'environ $20 \mathrm{~m}$. Son sommet qui présente un pendage non négligeable vers le sud est recouvert de lœss le rendant propice à la culture. Au pied de la butte, la dépression circulaire d'une centaine d'hectares est occupée par l'actuel marais du Grand-Plan, prolongé au sud par le marais lunifère de la Besseye. Son alimentation en eau est assurée en partie par les ruissellements et les résurgences suintant depuis la Butte-des-Vignes mais aussi par le Girondan qui le traverse de part en part. Le réseau de drainage moderne utilise aussi la rivière comme collecteur sur lequel il se branche grâce à un canal longeant le site archéologique au nord. Les couvertures aériennes du marais du Grand-Plan montrent qu'un exutoire existait dans sa partie nord-ouest (fig. 2). Creusé à l'Holocène par le Girondan qui coulait plus à l'est qu'aujourd'hui, il fut réaménagé dès l'Antiquité comme canal situé quelques mètres au nord de l'actuel. Le drainage alluvial naturel du marais a pu être bloqué par la formation d'une levée alluviale d'orientation sud-nord haute de 2 à $3 \mathrm{~m}$, antérieurement à la fin de l'âge du Bronze. Son point sommital, qui atteint la cote 202,20 m NGF, est à peu près au centre du site, aux abords immédiats de l'église. À l'ouest, elle est longée par le lit majeur du Girondan. Laccumulation de 


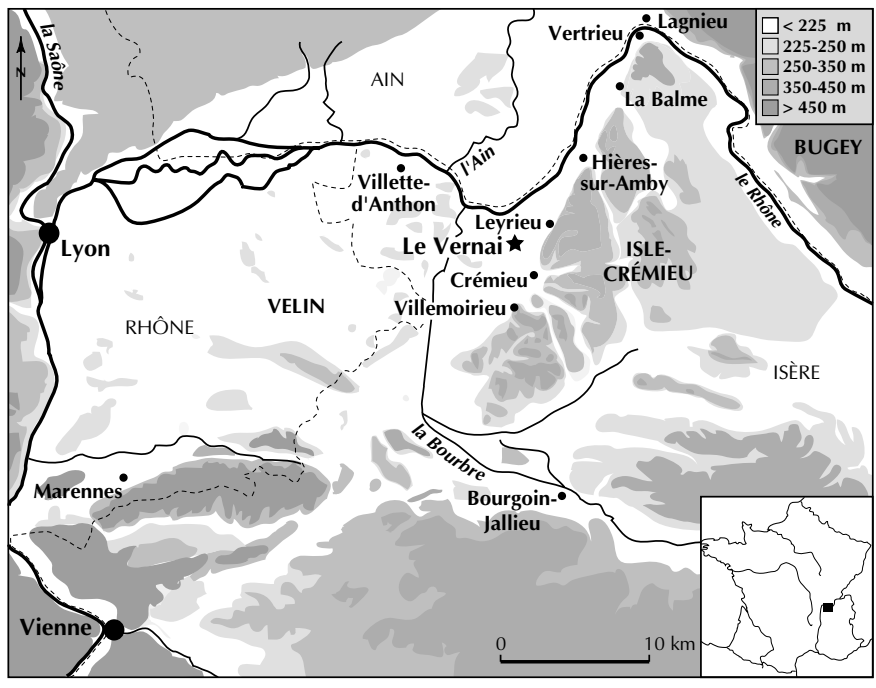

Fig. 1 - Localisation du site du Vernai à Saint-Romain-de-Jalionas, Isère (DAO R. Royet, SRA Rhône-Alpes).

dépôts de la rivière depuis l'âge du Bronze a d'ailleurs considérablement adouci la topographie de la partie occidentale du site. À l'est et au sud, ce microrelief est directement en contact avec le marais. Actuellement, ce replat limoneux s'étend au nord, pratiquement jusqu'au pied de la Buttedes-Vignes, mais il est possible qu'un paléochenal beaucoup plus marqué ait été remblayé pendant l'Antiquité.

Aujourd'hui, sur le site, se dresse l'église paroissiale entourée de sa cure et de son cimetière au milieu des prés. Les berges du Girondan sont occupées par une végétation herbacée hygro-hydrophile et arborée, associée à la forêt riveraine à peupliers, frênes et chênes. Le marais du GrandPlan n'est plus entretenu. Si les réseaux de drainages restent à peu près fonctionnels dans la partie nord-ouest, l'échec d'une tentative de peupleraie y a sonné la fin des cultures. Dans la moitié sud-est, le morcellement parcellaire a amené un abandon depuis plusieurs décennies ce qui, là encore, a eu pour conséquence une reconquête des taillis et d'une maigre forêt à chênes, hêtres et frênes.

\section{LE CONTEXTE ARCHÉOLOGIQUE ET HISTORIQUE}

À La Tène finale, l'Isle-Crémieu se situe à l'extrémité nord du territoire allobroge (fig. 3). Le Rhône marque vraisemblablement ici la frontière avec les Ambarres ou les Séquanes, même si l'incertitude subsiste encore sur le statut de la rive droite, César évoquant la détention par les Allobroges de vici et de possessiones au-delà du Rhône (César,
$B G, \mathrm{I}, 11)$. Une étude récente portant sur la répartition des épitaphes de l'aristocratie gallo-romaine d'origine allobroge limite toutefois ces enclaves outre-fleuve à de simples têtes de pont (Rémy, 2000).

Les axes de communication protohistoriques restent mal connus, mais leur réseau est induit par les gués du Rhône parmi lesquels celui de Saint-Oyand, au nord de la commune, et celui du Port du Noyer à La Balme-lesGrottes (Royet, Faucher, 2002), situé à $3 \mathrm{~km}$ en amont (fig. 4). Pour cette époque il semble acquis qu'une route remontait la vallée en longeant le rebord de la moyenne terrasse du Rhône. Sur cet axe se branchaient certainement des chemins perpendiculaires assurant la remontée sur le plateau en empruntant les vaux de la Fusa, de l'Amby et d'Amblérieu. L'importance de ces pénétrantes reste à évaluer, et il n'est pas évident qu'elles aient été le support d'un commerce interrégional. Seule une voie contournant le plateau par le sud, via le val de la Fusa, constitue un réel raccourci à une route suivant le fleuve.

Cette partie de la vallée du Rhône est alors contrôlée par l'oppidum de Larina juché sur le rebord du plateau de l'Isle-Crémieu à Hières-sur-Amby. Si son enceinte enserre près de 27 ha, il n'a toutefois pas regroupé toute la population locale en son sein. Au contraire, les prospections montrent que la vallée a connu un peuplement important mais inégalement réparti. Deux types d'emplacements attirent préférentiellement les implantations. Les fermes, souvent à l'origine de villae, sont installées essentiellement au bord des dépressions humides. On trouve aussi, espacés à peu près tous les kilomètres sur la basse terrasse du fleuve, des sites de berge couvrant environ 1 ha (Royet, Faucher, 2002). La terrasse fersiallitique paraît peu attractive pour l'agriculture. À Saint-Romain-de-Jalionas, elle n'est occupée que par une vaste nécropole.

Après la conquête romaine l'Isle-Crémieu fait partie de la cité de Vienne, mais elle paraît aussi, à partir de l'époque augustéenne, être intégrée dans la sphère d'influence économique de la colonie de Lyon. La carte des épitaphes retrouvées le long du Rhône montre que des notables lyonnais et viennois possèdaient alors des domaines sur les deux rives, indépendamment du découpage administratif.

L'organisation du réseau routier, particulièrement dense, atteste à la fois cette porosité des limites entre les provinces de Lyonnaise et de Narbonnaise, et l'importance du nœud de communication que représente alors cette portion de vallée (fig. 4). Les voies s'organisent en grande partie autour du gué de Saint-Oyand, mais les divagations du cours de l'Ain depuis l'Antiquité ont effacé celles qui convergeaient 


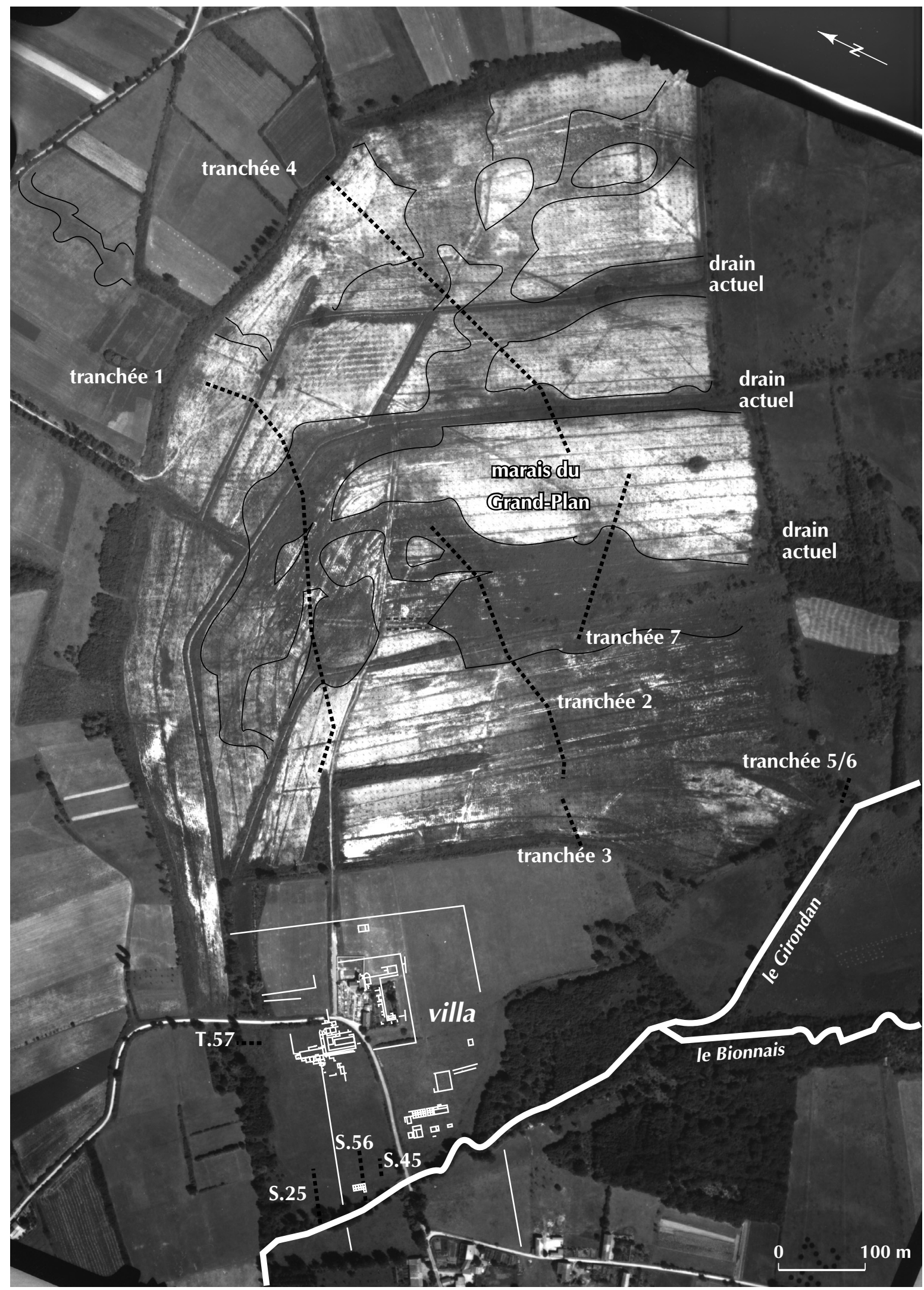

Fig. 2 - Localisation des sondages sur le site du Vernai et le marais du Grand-Plan (photo-interprétation J.-F. Berger, CNRS). 


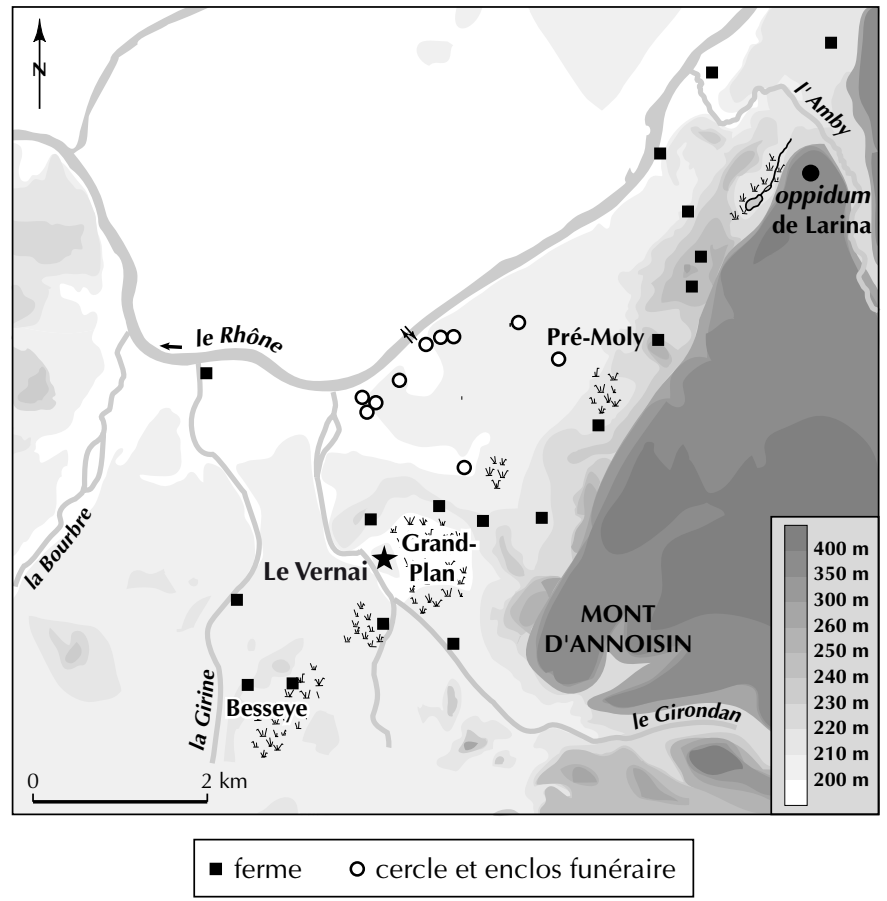

Fig. 3 - Occupation laténienne en Isère sur les communes de SaintRomain-de-Jalionas, Leyrieu, Vernas et Hières-sur-Amby (DAO R. Royet, SRA Rhône-Alpes).

depuis l'ouest sur la rive droite, notamment la route dite de Suisse par la rive droite (Buisson, Pelletier, 1983). Seule une route se dirigeant vers le nord-est et passant par l'agglomération de Saint-Vulbas, en direction du vicus d'Ambérieu, se lit sur les photographies aériennes.

Le raccordement direct entre Vienne et la rive gauche du haut-Rhône est assuré par une voie dite de Saint-Oyand, dont le tracé fossile peut être suivi sur toute la commune de Saint-Romain-de-Jalionas puis, au-delà, reconstitué par le raccordement de chemins ruraux actuels. Elle est pratiquement rectiligne entre le gué de Saint-Oyand et son raccordement au niveau de la commune de Satolas-etBonce, sur la voie d'Agrippa vers l'Italie dont le tracé est, grosso modo, repris par la RN6.

La liaison avec Lyon était possible par deux itinéraires. La route la plus directe se raccorde à l'axe précédemment évoqué au niveau du gué de Saint-Oyand. Au-delà du Girondan, elle semble se diriger vers l'ouest mais, traversant un environnement trop urbanisé, il est rapidement impossible de la suivre. Le deuxième itinéraire reconnu correspond à un compendium entre Lyon et la voie de Suisse. Cet axe mal identifié longeait la rive du Rhône depuis Lyon jusqu'au gué de Villette-d'Anthon. Cette route traversait Saint-Romain-

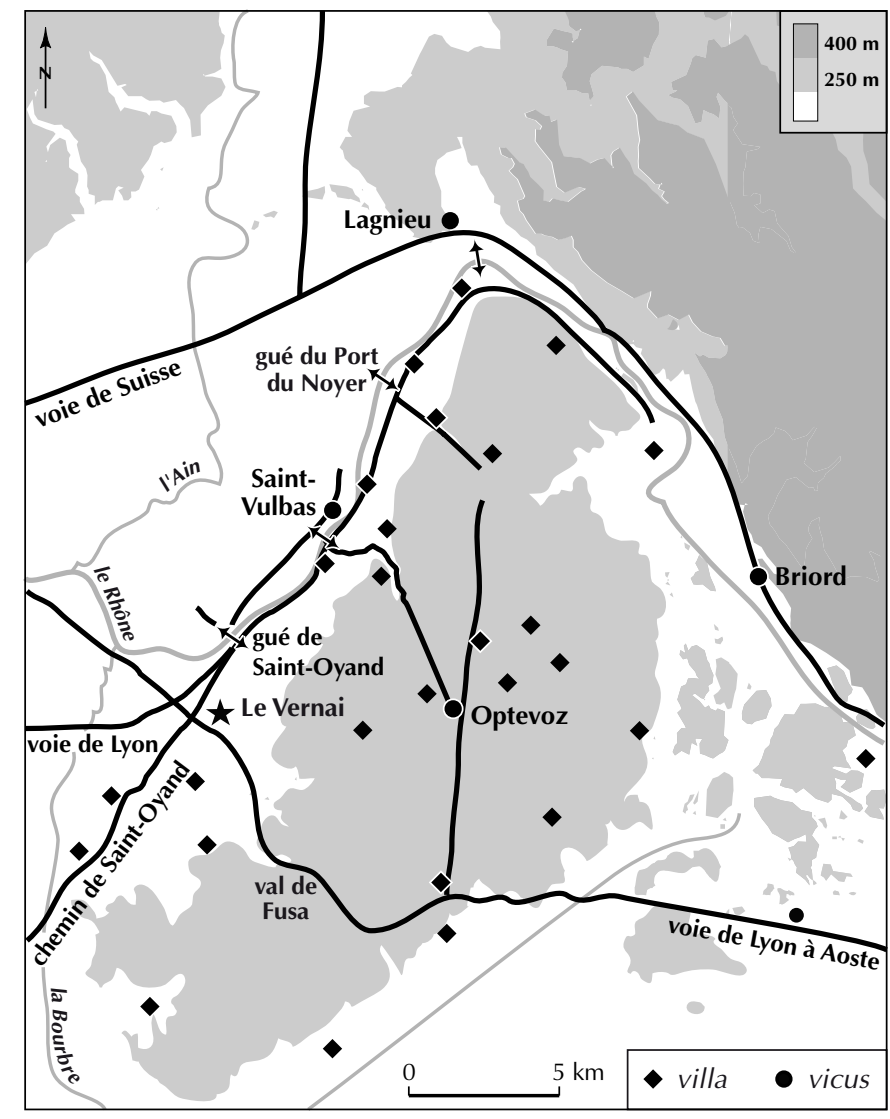

Fig. 4 - Voies de communication et passages du Rhône autour de l'Isle-Crémieu pendant l'Antiquité (DAO R. Royet, SRA RhôneAlpes).

de-Jalionas selon une direction nord-ouest-sud-est. Elle rejoignait ensuite la voie de Suisse après avoir emprunté le val de la Fusa afin de couper au plus court au travers de l'Isle-Crémieu. Le site du Vernai se trouve en retrait de cet axe majeur auquel il était relié par un diverticule parallèle à l'actuelle route vicinale qui traverse les vestiges (voir infra, p. 302).

$\mathrm{Au}$ contraire de la rive droite, on ne connaît pas d'agglomération secondaire dans la vallée sur $80 \mathrm{~km}$ entre Lyon et Aoste. Des prospections systématiques ont conduit récemment à réinterpréter le secteur de Travers à La Balmeles-Grottes où l'historiographie localisait un vicus (Royet, Faucher, 2002). Aujourd'hui, seule peut être retenue l'agglomération d'Optevoz située au centre du plateau (Royet, Coquidé, à paraître). On peut, en revanche, restituer dès l'époque tibéro-claudienne un réseau de grandes villae d'une taille et d'une fréquence inconnues dans le Velin ou même plus en amont, au-delà de Sault-Brénaz, où les fermes représentent le type d'établissement le plus fréquent. Les 
critères de choix présidant à leur localisation paraissent plus complexes que pour la période antérieure. Outre les sites déjà occupés à la fin de La Tène, on constate des implantations le long des voies (villa de Vertrieu) ou en bordure du Rhône (Sainte-Colombe à La Balme-lesGrottes). Dans la plaine, entre Saint-Romain-de-Jalionas et Vertrieu, les prospections mettent en évidence un site interprété comme une villa tous les $2 \mathrm{~km}$ en moyenne. Ces grands établissements vont souvent constituer un pôle structurant stable pendant toute l'Antiquité. Seuls quelques rares cas d'abandon précoce de villae (villa de Sainte-Colombe) peuvent être signalés au contraire des fermes qui sont souvent désertées puis, éventuellement, partiellement réoccupées au $\mathrm{IV}^{\mathrm{e}} \mathrm{s}$. Après l'acmé de la fin du $\mathrm{I}^{\mathrm{er}}$ s., la désertion progressive d'une partie des sites n'est ni linéaire ni réductible à quelques événements ou catastrophes. Ainsi, à la fin de l'Antiquité on assiste non seulement à une concentration au profit des plus grandes villae comme Saint-Romain-deJalionas, similaire à ce qui est constaté partout en Gaule du Sud, mais aussi à un redéploiement de petits habitats ou ateliers, notamment autour des zones humides, souvent sur des lieux abandonnés plusieurs siècles auparavant.

Avec la création de la Sapaudia puis sa prise de contrôle par les Francs à partir de 534, la partie ouest de l'IsleCrémieu, aux confins des diocèses de Lyon et de Vienne, n'occupe ainsi qu'une position périphérique. Le Rhône garde, jusqu'à Hières-sur-Amby, une fonction de limite de diocèse.

L'effondrement du cadre administratif antique n'a pas remis fondamentalement en cause la carte des implantations aristocratiques. La création d'un nouveau domaine sur Larina représente une exception dans cette microrégion. $\mathrm{Au}$ début $\mathrm{du} \mathrm{VI}^{\mathrm{e}} \mathrm{s}$. le rempart de l'ancien oppidum est restauré et une grande résidence construite ex nihilo. Il faut peut-être voir dans ce cas l'illustration de l'installation d'une nouvelle aristocratie dans des propriétés issues du démembrement des domaines de la fin de l'Antiquité en application des dispositions de la loi Gombette (Porte, 2002). La création de cet habitat fortifié n'illustre toutefois pas un repli généralisé sur les hauteurs. En effet, l'occupation du sol dans la plaine ne semble généralement pas bouleversée. Les mêmes terroirs restent mis en valeur, même si la redistribution d'une partie de la population est sensible. Des sites intermédiaires au statut incertain, associant l'habitat et son cimetière mais dépourvus d'église, apparaissent. En l'absence de fouilles, dans l'Isle-Crémieu et à plus grande échelle dans le Velin, on s'interroge sur la permanence de grands domaines pérennisant des villae antiques (Faure-Boucharlat dir., 2001). Les sites des villae de Marignieu à Hières-sur-Amby et de Sainte-Marie-de-Tortas à Leyrieu restent fréquentés sans que l'on puisse assurer la réelle permanence d'une résidence aristocratique. Sur la base de prospections, faute de marqueur permettant l'identification d'un habitat de qualité, il est difficile d'exclure qu'un habitat paysan regroupé n'ait succédé à une exploitation domaniale.

\section{HISTORIQUE ET PROBLÉMATIQUE DES RECHERGHES}

\section{LES FOUILLES ANCIENNES}

L'exhumation de débris gallo-romains autour de l'église fut signalée dès la fin du XIX ${ }^{\mathrm{e}} \mathrm{s}$. (Lacroix, 1883) et des excavations à la recherche d'un trésor enfoui eurent lieu vers 1920 dans la parcelle AH30. Ce n'est toutefois qu'en 1967 que le site fut redécouvert et que furent déclenchées les premières fouilles fournissant plans et rapports. Pendant douze ans, les chercheurs successifs, R. Pinet puis A. Dufourg, ouvrirent trois chantiers principaux fouillant un ensemble balnéaire (fig. 5, $\mathrm{n}^{\mathrm{o}} 1$ et fig. 6), plusieurs bâtiments de la pars rustica (fig. 5, $\mathrm{n}^{\circ} 2$ et fig. 19), puis vraisemblablement l'extrémité méridionale de la partie résidentielle (fig. 5, no 3 et fig. 6).

En 1985, l'extension du cimetière communal sur $1500 \mathrm{~m}^{2}$ dans la parcelle AI181, au sud de l'église (fig. 5, no 4), donna lieu à une fouille de sauvetage.

Depuis, plusieurs sondages préalables à des opérations d'urbanisme ont donné l'occasion de compléter ces informations en précisant les limites occidentales du site. En 1986, l'angle sud-ouest du mur de délimitation de la villa fut repéré lors d'un projet de lotissement (fig. 5, no 5). Enfin, en 1999, un bâtiment antique fut relevé dans la parcelle AO99 lors la construction d'un pavillon (inédit) (fig. 5, $\mathrm{n}^{\mathrm{o}} 7$ ).

\section{LES TRAVAUX ACTUELS}

Les études relancées depuis 1994 visent à comprendre l'organisation et la stratigraphie du gisement, mais aussi à appréhender la dialectique entre le site et son environnement.

Les premiers travaux sur l'habitat ont d'abord essentiellement cherché à recaler et à réinterpréter les fouilles anciennes, ainsi qu'à procéder à une évaluation détaillée de la parcelle AH30 qui couvre environ 2 ha. Depuis 1998, la fouille exhaustive de AH30 a été engagée. À ce jour 


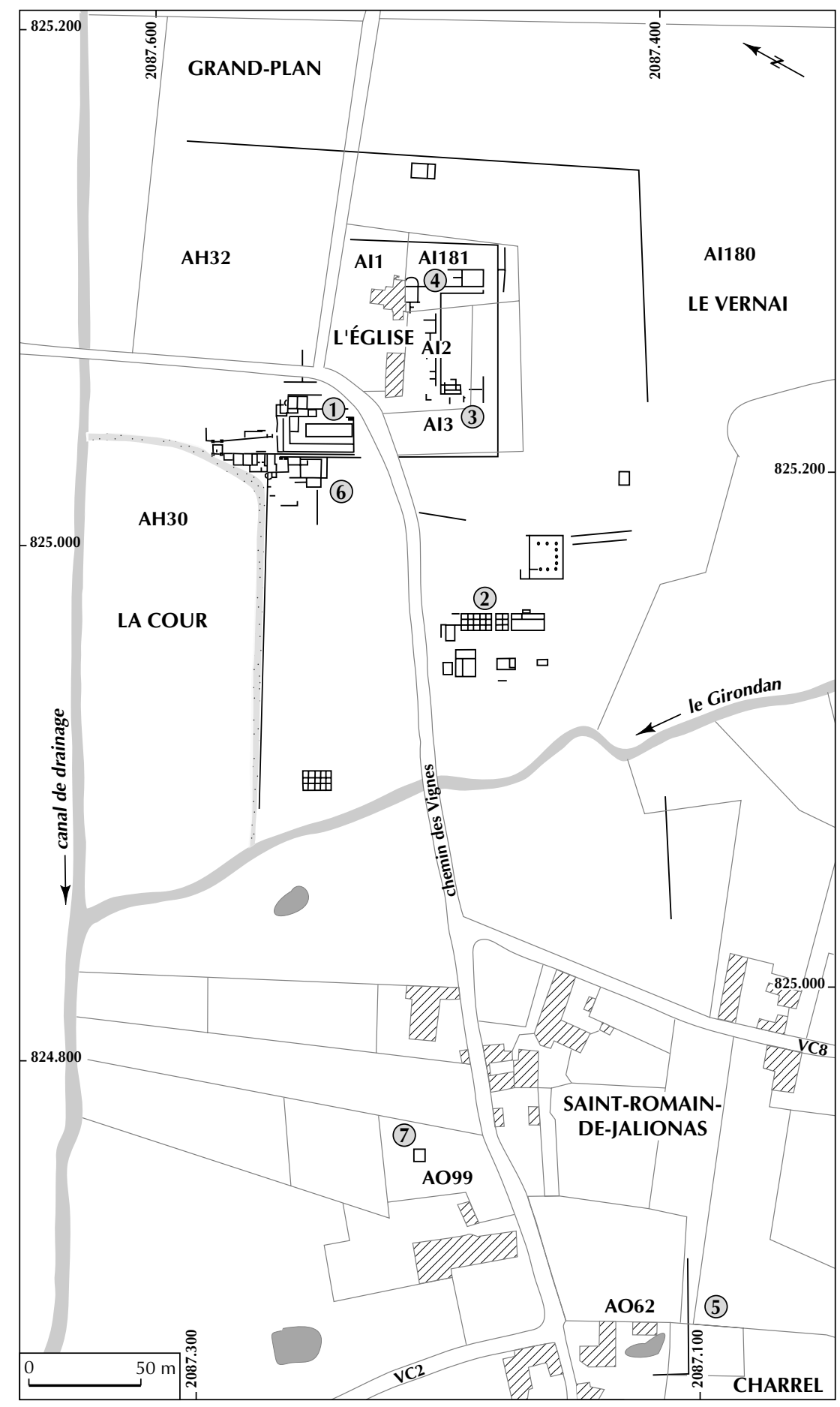

VZ bâti contemporain

(1) numéro des chantiers archéologiques

épandage de matériaux antiques

Fig. 5 - Plan topographique de la villa du Vernai du $I^{e r}$ au $V^{e} s$. (DAO R. Royet, SRA Rhône-Alpes). 


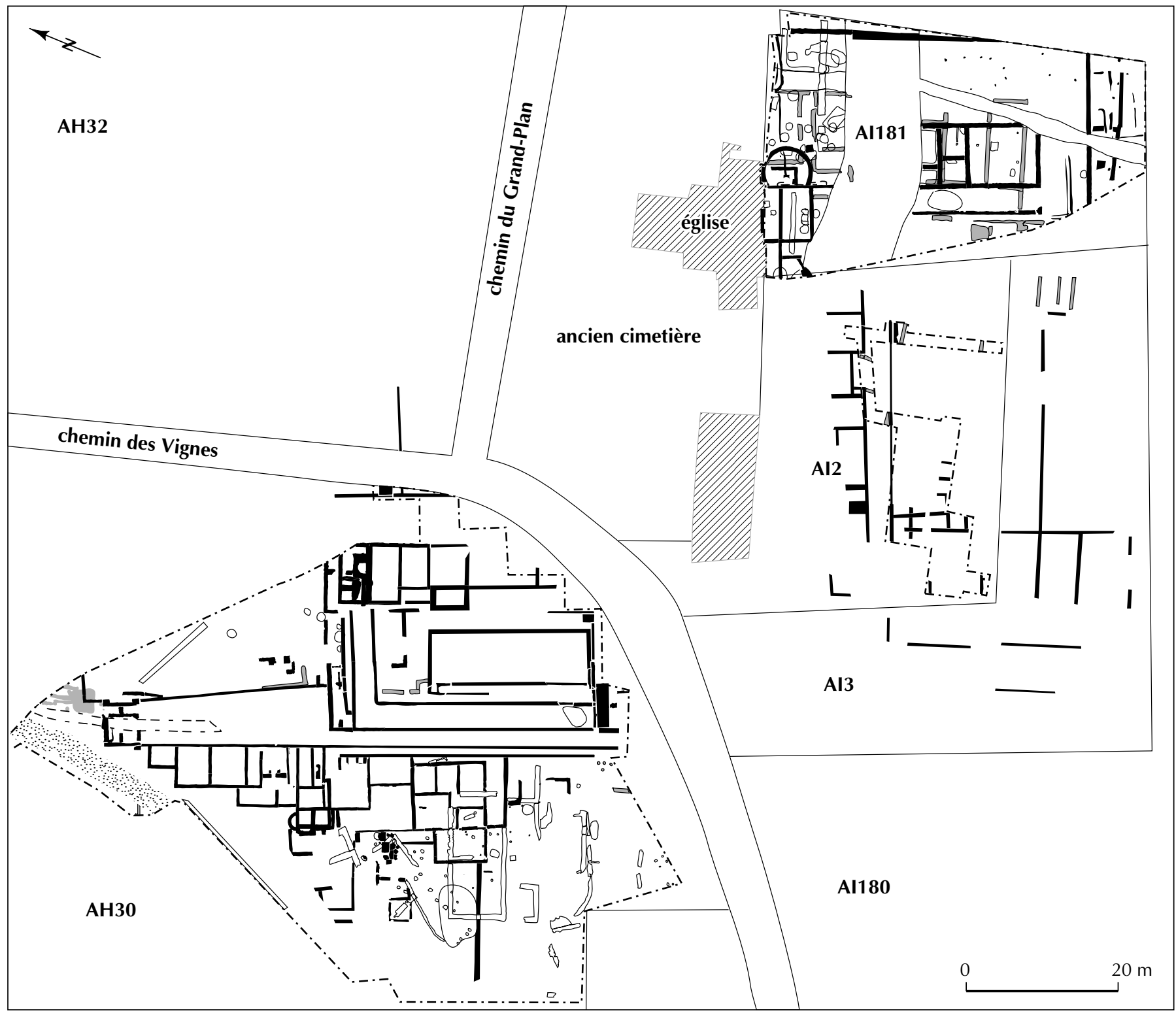

partie résidentielle $\square$ première villa $\square$ bâti contemporain $\quad \square$ autre période $\quad$-.- limite de fouille

Fig. 6 - Parcelles AH30, AI2 et AII81 du site du Vernai (DAO R. Royet, SRA Rhône-Alpes).

l'étude a essentiellement porté sur les occupations postérieures à l'Antiquité. La stratification de la villa a été surtout étudiée dans les tranchées d'évaluation et dans quelques fenêtres limitées. Exception faite de l'extrémité nord de la pars urbana et d'un bâtiment isolé fouillé sur les bords du Girondan (secteur XX), seuls les niveaux de l'Antiquité tardive mis au jour lors du démontage des occupations médiévales ont été étudiés (fig. 6). Malgré l'importance des surfaces décapées (près de $4000 \mathrm{~m}^{2}$ ), la connaissance des occupations anciennes sur ce site demeure ainsi encore très lacunaire et repose essentiellement sur la fouille de sauvetage de 1985. La partie économique de la villa reste particulièrement mal connue.

Les terrains hydromorphes situés en bordure de la villa et considérés comme le conservatoire des évolutions morphologiques, hydrologiques et économiques font l'objet d'un examen attentif depuis 1997. La reconnaissance des évolutions du marais du Grand-Plan, au-delà des contraintes 
sanitaires qu'il impose à l'habitat, est notamment rapidement apparue essentielle pour la compréhension du fonctionnement de l'exploitation agricole gallo-romaine. Au total, sept tranchées, d'une longueur cumulée d'environ $1500 \mathrm{~m}$, ont été ouvertes dans sa moitié nord-ouest (fig. 2). Les berges de la rivière bordant le site ont, elles aussi, été recoupées à quatre reprises. Un travail de description et de caractérisation systématique des faciès pédosédimentaires a été réalisé sur le terrain, afin d'établir des corrélations entre les sondages et de construire une typologie des faciès de remplissage des structures agraires enterrées (Berger, 2001). En effet, chaque faciès de colmatage des fossés bordiers correspond à un état de fonctionnement du réseau hydraulique et des parcelles qui l'encadrent. L'ensemble des faciès reconnus a fait l'objet d'un échantillonnage géoarchéologique et paléobotanique dans le but de reconstituer les paléovégétations et d'identifier les traces de cultures, ainsi que les variations hydrologiques et climatiques.

Ce travail, hors site, sur l'environnement et le terroir de la villa est complété par des prospections systématiques réitérées dans ce tronçon de la vallée du Rhône (fig. 3, 12 et 22).

\section{LES ÉTAPES DE L'OCCUPATION}

\section{LE SITE LATÉNIEN}

Une occupation pérenne précédant l'établissement gallo-romain est attestée sur le site du Vernai et dans le marais du Grand-Plan (fig. 7).

\section{EMPRISE DE L'HABITAT}

Les témoins stratifiés laténiens ont été retrouvés sur la partie la plus haute de la levée alluviale (parcelle AI181), à une altitude variant entre 202,30 m et 202,40 m NGF. Un niveau de fréquentation a été reconnu par sondages sur environ $2000 \mathrm{~m}^{2}$ dans les parcelles AI181 et AI2, mais n'a pu être fouillé que sur environ $50 \mathrm{~m}^{2}$. L'organisation des vestiges immobiliers est difficile à restituer (fig. 8). Les trous de poteaux et de piquets présentent des formes variées et ne peuvent être alignés. Plutôt qu'un bâtiment, on peut proposer de voir dans ce secteur des aires de travail éventuellement protégées des intempéries par des palissades. Le matériel associé ne permet toutefois pas de préciser la nature des activités.

Si toutes les structures sont installées dans le même horizon datable du milieu du ${ }^{\mathrm{er}}$ s. av. J.-C., la stratigraphie

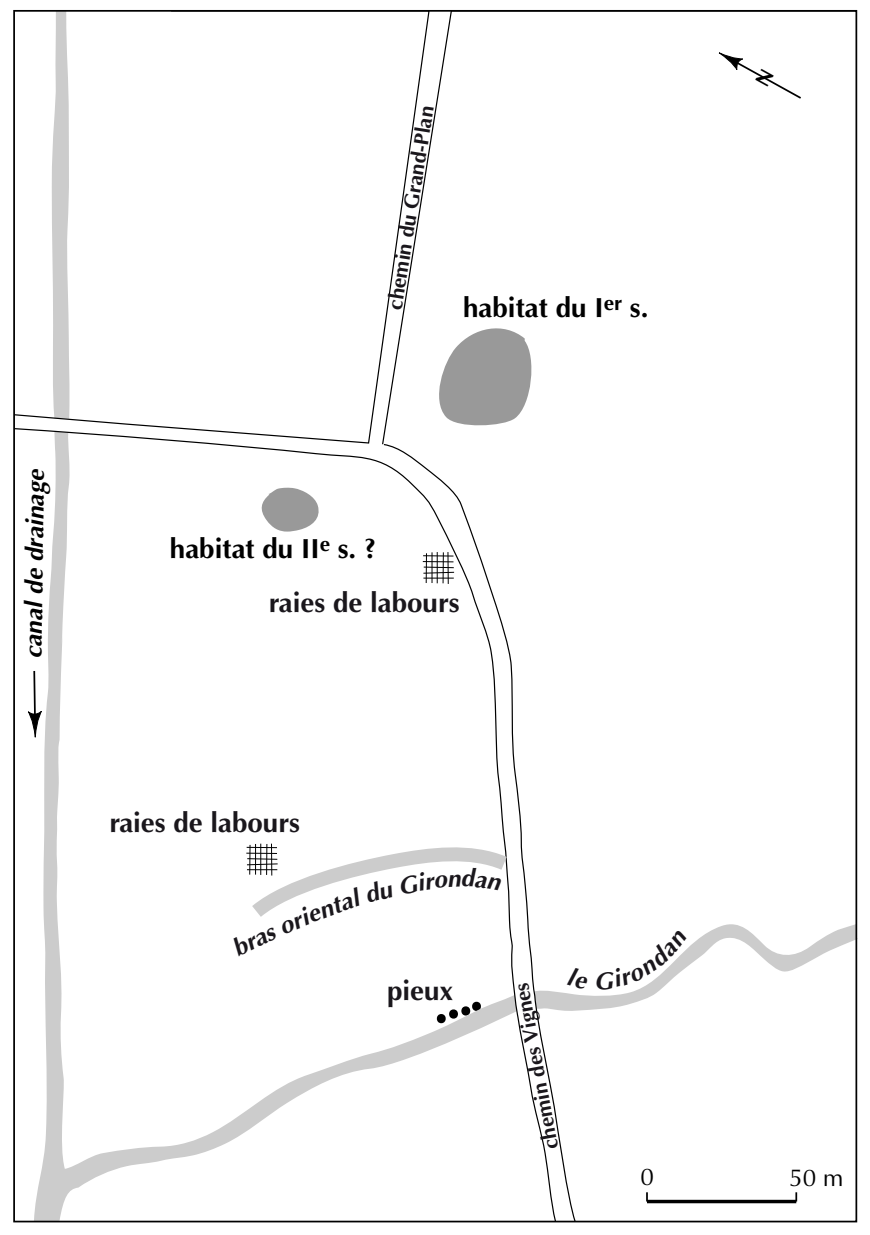

Fig. 7 - Plan général du site du Vernai à La Tène (DAO R. Royet, SRA Rhône-Alpes).

montre que l'occupation s'est étendue dans le temps. Deux niveaux de sols superposés ont été différenciés.

L'hypothèse d'une occupation laténienne de longue durée est suggérée par des découvertes éparses sur le site. Les fouilles de 1967 avaient exhumé, hors de tout contexte stratigraphique, des tessons de céramique d'ensembles datant des $\mathrm{III}^{\mathrm{e}}$ et $\mathrm{II}^{\mathrm{e}}$ s. av. J.-C. Ce matériel peu fragmenté proviendrait de fosse(s) située(s) à environ $100 \mathrm{~m}$ au nordouest des vestiges plus récents.

\section{LES ZONES HUMIDES : LE LIT MAJEUR DU GIRONDAN ET LE GRAND-PLAN}

Si l'aménagement de la butte alluviale n'a pas impliqué de travaux particuliers, le contrôle du lit du Girondan paraît avoir été plus délicat. Les efforts consentis pour y parvenir restent difficiles à appréhender. Un alignement de 


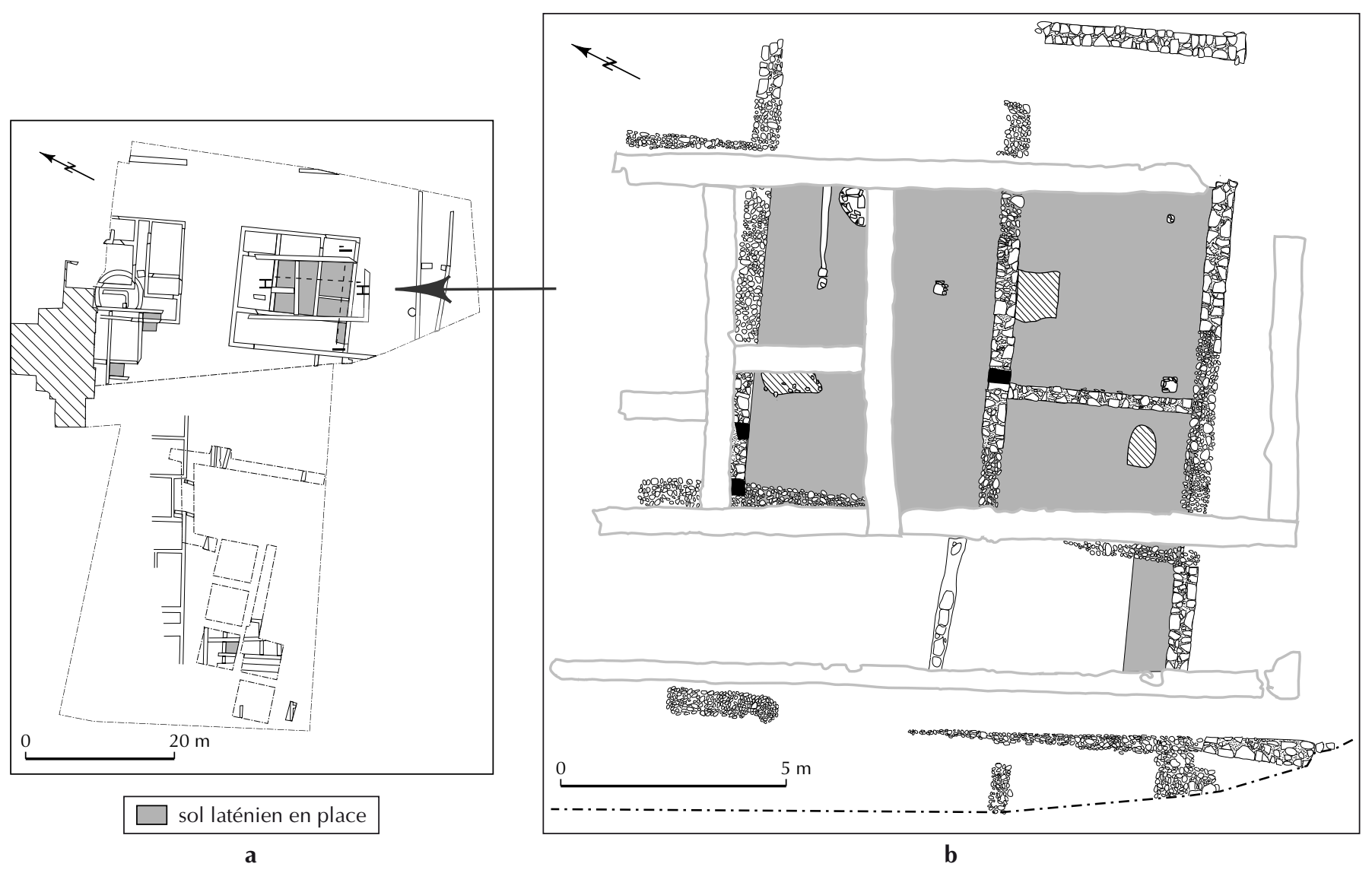

Fig. 8 - Vestiges fouillés sur le sommet de la butte du site du Vernai:

a, localisation des niveaux laténiens (AI4 et AII81); b, niveaux laténiens sous la première villa (DAO R. Royet, SRA Rhône-Alpes).

piquets de chêne, espacés d'une quarantaine de centimètres et fichés dans le lit du Girondan, témoigne d'une tentative de stabilisation de la berge de la rivière contemporaine des plus anciens témoins d'occupation (2180 $\pm 40 \mathrm{BP})$ (fig. 7). On peut s'interroger sur l'utilité de cette protection de la rive alors que le lit de la rivière était profondément encaissé et que sa plaine d'inondation ne connaissait qu'un usage agricole. La présence encore massive d'aulnes et de plantes hygrophiles dans les diagrammes polliniques suggère d'ailleurs la conservation, au moins partielle, de la ripisylve (fig. 9 et 10).

La période de calme hydrologique s'interrompt à la fin du II $^{\mathrm{e}} \mathrm{s}$. ou au début du I ${ }^{\mathrm{er}} \mathrm{s}$. av. J.-C. La rivière connaît alors un régime torrentiel associé à des crues de haute énergie (dépôts dominés par les sables fins) qui se traduit par le déblaiement de niveaux archéologiques et la progradation d'une charge sablo-graveleuse riche en matériel céramique et en débris de bois relevant le fond du lit de plus de $0,80 \mathrm{~m}$ (pl. VIII hors texte). La submersion des aménagements des berges entraîne un déplacement du lit de la rivière vers l'est ou, plus vraisemblablement, un dédoublement de son cours. Plusieurs sondages situés en divers points de la plaine du Girondan montrent en effet l'existence d'un bras oriental à près de $40 \mathrm{~m}$ à l'est du cours actuel (fig. 7). Un alignement de poteaux trouvé en bordure du lit mineur peut témoigner d'une tentative infructueuse de stabilisation. La date radiocarbone effectuée sur un pieu (2050 $\pm 50 \mathrm{BP}$ ) n'exclut d'ailleurs pas que cet aménagement ait été immédiatement postérieur à cette phase de l'occupation du site et ne corresponde en fait à la phase initiale d'aménagement gallo-romaine.

À l'extérieur du site, les informations - encore limitées permettent d'envisager une emprise de cette exploitation sur un environnement assez étendu incluant notamment les terres humides. Actuellement, un fossé (T.4 m.172), recoupé dans la partie centrale du marais, peut être raccordé à cette phase de mise en valeur du marais du Grand-Plan, grâce à la datation d'un lit de grains d'orge carbonisés 


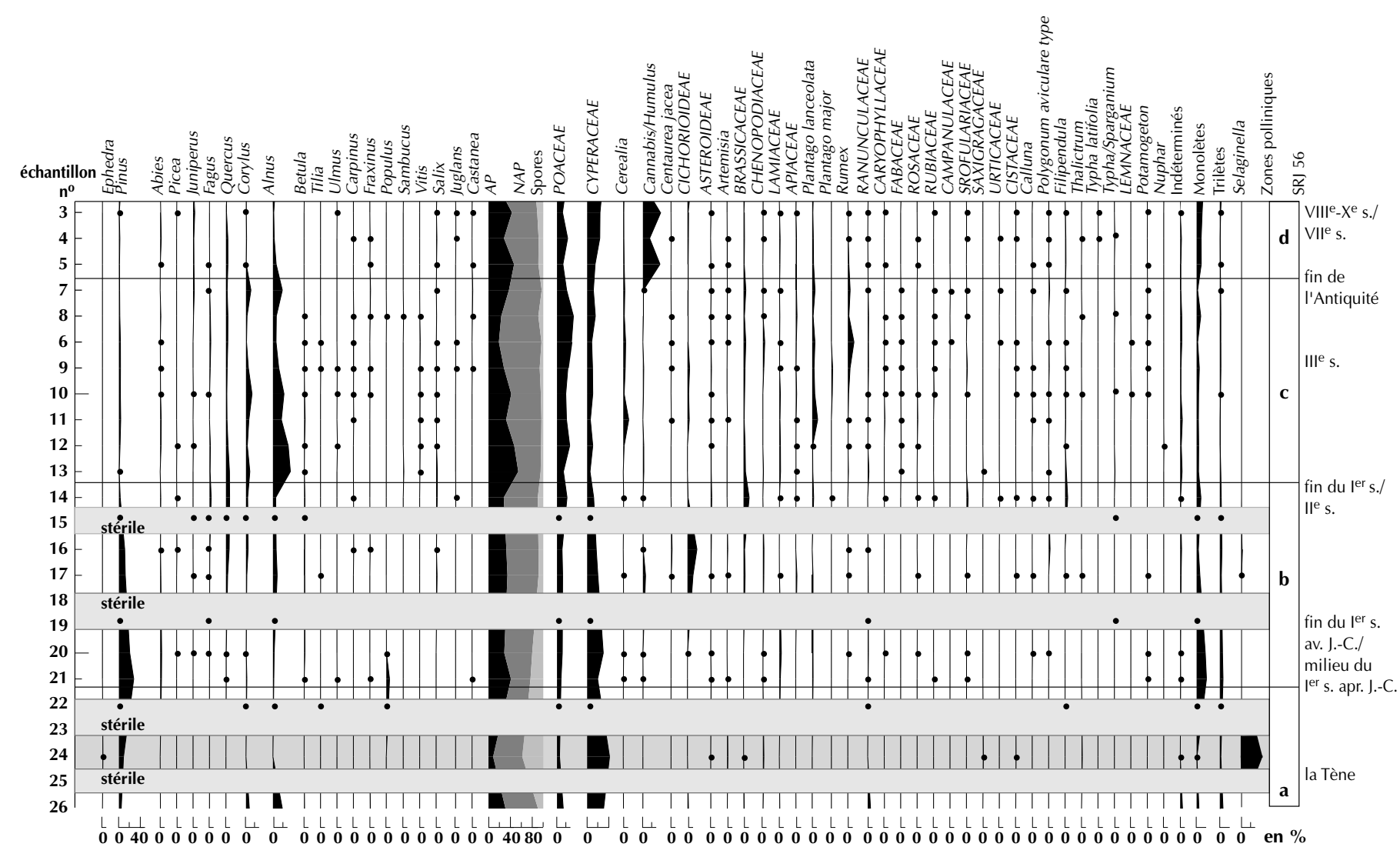

Fig. 9 - Diagramme pollinique des berges du Girondan (T.56, voir fig. 2) (DAO J. Argant, CNRS).

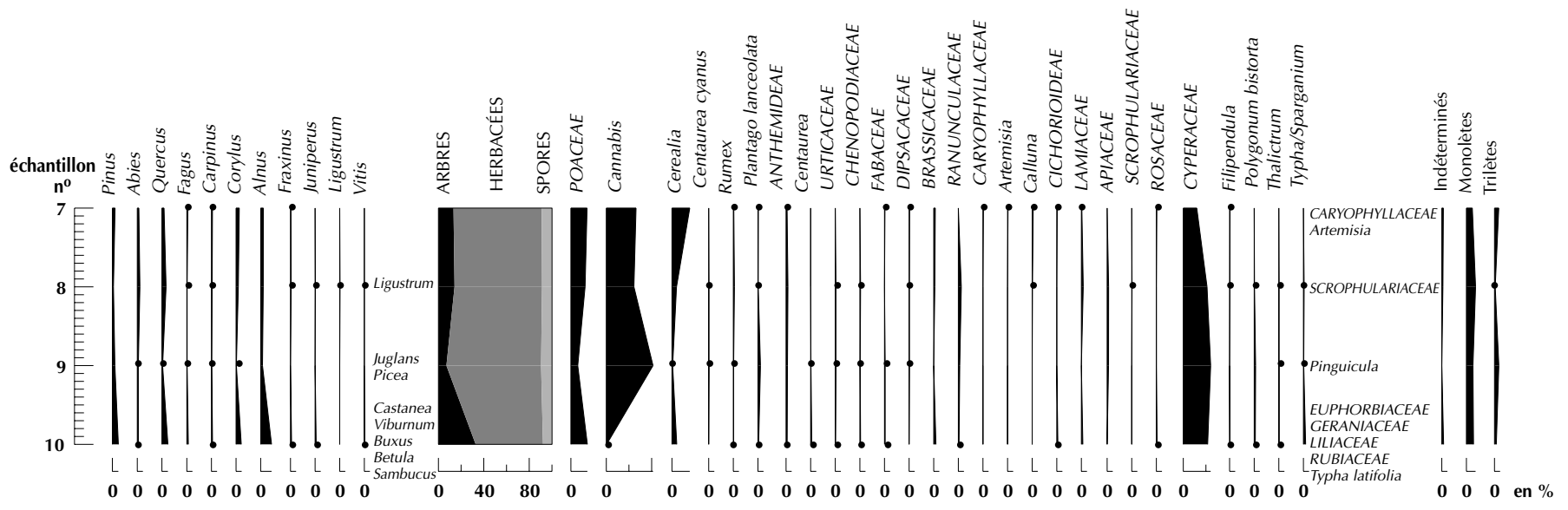

Fig. 10 - Diagramme pollinique des berges du Girondan (T.45, voir fig. 2) (DAO J. Argant, CNRS). 
Tabl. I - Faune. Nombre de restes par taxon et par période: av. $1^{r e} v$., avant la $1^{r e}$ villa; $1^{e} v$., villa précoce; $1^{e} / 2^{e} v .1^{e}$, niveaux du $I^{e r}$ s. apr. J.-C. appartenant à la villa précoce ou à la seconde villa; $2^{e} v$. he, niveaux appartenant à la seconde villa et datant du $I^{e r}$ ou II ${ }^{e}$ s. apr. J.-C; 2ef, fin du II s. apr.J.-C.; at/fa, Antiquité tardive/fin de l'Antiquité; les autres catégories correspondent à des divisions par siècles.

\begin{tabular}{|c|c|c|c|c|c|c|c|c|c|c|c|c|c|c|c|}
\hline Taxon Période & $\begin{array}{l}\text { av. } \\
1^{\text {re }} \text { v. }\end{array}$ & $1^{e} v$. & $\begin{array}{c}1 \mathrm{e} / 2^{\mathrm{e}} \mathrm{v} . \\
1^{\mathrm{e}}\end{array}$ & $\begin{array}{l}2^{e} v . \\
\text { he }\end{array}$ & $1 \mathrm{e}$ & $1^{e} / 2^{e}$ & $2^{e}$ & 2 ef & $2^{e} / 3^{e}$ & $3^{\mathbf{e}}$ & $3^{e} / 4^{e}$ & $4^{e}$ & $4^{e} / 5^{e}$ & $5^{e}$ & at $/ \mathbf{f a}$ \\
\hline Ovicaprin & 2 & 41 & & & 11 & 5 & 68 & 25 & 51 & 49 & 2 & 57 & 120 & 29 & 7 \\
\hline Porcin & 12 & 203 & 3 & 2 & 50 & 25 & 467 & 66 & 291 & 221 & 39 & 329 & 182 & 185 & 19 \\
\hline Bovin & 2 & 71 & 1 & 2 & 23 & 5 & 208 & 29 & 179 & 178 & 19 & 201 & 154 & 156 & 15 \\
\hline Équidé & & 16 & & & 2 & & 12 & & 9 & 5 & 5 & 14 & 14 & 5 & 2 \\
\hline Cerf & & 21 & & & 6 & & 33 & 3 & 36 & 36 & 4 & 64 & 14 & 30 & \\
\hline Chevreuil & & & & & & & 1 & 1 & & & & 1 & & & \\
\hline Chien & & 6 & & & 5 & & 1 & 3 & 20 & 5 & 6 & 6 & 16 & 3 & \\
\hline Renard & & & & & & & & & 2 & & & & & & \\
\hline Chat & & & & & & & & & 5 & & & & & & 1 \\
\hline Blaireau & & & & & & & & & & & & 1 & & & \\
\hline Lièvre & 1 & 19 & & & 3 & 1 & 18 & 6 & 9 & 3 & 1 & 3 & 6 & 2 & \\
\hline Lapin & & & & & & & & & 1 & & 1 & & & & \\
\hline Castor & & & & & & & 1 & & & & & & 1 & & \\
\hline Marmotte & & & & & & & 1 & & & & & & & & \\
\hline Micromammifère & & & & & & & 1 & & & 3 & & & & & \\
\hline Oiseau & 1 & 88 & 2 & 1 & 15 & 8 & 183 & 29 & 127 & 44 & 9 & 73 & 12 & 37 & 10 \\
\hline Tortue d'eau & & 1 & & & & & 2 & 1 & 1 & 7 & & & & & \\
\hline Poisson & & 2 & & & & & & & & & & & & & \\
\hline Total NR & 18 & 468 & 6 & 5 & 115 & 44 & 996 & 163 & 731 & 551 & 86 & 749 & 519 & 447 & 54 \\
\hline \multicolumn{16}{|l|}{ VERTÈBRES } \\
\hline Ovicaprin & & 1 & & & & & & & 1 & & & & 2 & 1 & \\
\hline Porcin & 1 & 18 & & & & 1 & 18 & & 11 & 1 & & 5 & 21 & 3 & 1 \\
\hline Bovin & & 6 & & 1 & 1 & & 11 & 4 & 8 & 10 & 4 & 12 & 7 & 9 & \\
\hline Total & 1 & 25 & & 1 & 1 & 1 & 29 & 4 & 20 & 11 & 4 & 17 & 30 & 13 & 1 \\
\hline \multicolumn{16}{|l|}{ CÔTES } \\
\hline Ovicaprin & & 7 & & & 1 & & 5 & 1 & 2 & 1 & 1 & 1 & 35 & 2 & \\
\hline Porcin & 4 & 55 & & 3 & 4 & & 70 & 11 & 53 & 21 & 4 & 48 & 25 & 38 & 2 \\
\hline Bovin & 1 & 14 & & & 1 & & 28 & 9 & 14 & 10 & 2 & 37 & 24 & 88 & 2 \\
\hline Total & 5 & 76 & & 3 & 6 & & 103 & 21 & 69 & 32 & 7 & 86 & 84 & 128 & 4 \\
\hline Déterminé & 24 & 569 & 6 & 9 & 122 & 45 & 1128 & 188 & 820 & 594 & 97 & 852 & 633 & 588 & 59 \\
\hline Indéterminé & 16 & 551 & 7 & 10 & 71 & 32 & 856 & 187 & 716 & 619 & 129 & 703 & 165 & 223 & 57 \\
\hline Total restes & 40 & 1120 & 13 & 19 & 193 & 77 & 1984 & 375 & 1536 & 1213 & 226 & 1555 & 798 & 811 & 116 \\
\hline
\end{tabular}

échantillonné au sein de son remplissage sableux microlaminé $(2260 \pm 70 \mathrm{BP})$. Ce vestige encore isolé témoigne ainsi d'un aménagement, au moins partiel, du marais du Grand-Plan.

Il est certain que pour être cultivée, la dépression du Grand-Plan dut en effet être drainée à la fin de La Tène. Le paléosol gris supportant l'installation du premier fossé présente une texture limoneuse à sableuse et une structure massive à porosité fermée qui indiquent une hydromorphie prolongée (processus de gleyfication). L'organisation du réseau drainant protohistorique ne peut toutefois être reconstituée actuellement. Il se raccordait vraisemblablement sur le Girondan en aval mais aussi en amont du marais. Le remplissage du fossé laténien est en effet 
constitué d'un microlitage de sables et limons exogènes au marais évoquant plutôt une irrigation qu'un drainage.

\section{LES PRODUCTIONS}

Le témoignage d'une activité agricole a pu être attesté en différents points de la clairière par la mise en évidence des traces de labours croisés sur un paléosol fluvial, consécutif à un écobuage, antérieur à la première villa (fig. 7 et 13). Celles-ci sont présentes non seulement dans le limon de la levée alluviale (sondage 10), à proximité des constructions gallo-romaines les plus anciennes, mais aussi dans le fluviosol sablonneux grisâtre de la dépression du lit majeur du Girondan. Faute de macrorestes et les tests palynologiques s'étant révélés négatifs, il n'est pas possible de déterminer les espèces cultivées. En revanche, quelques indices ont été recueillis en périphérie du site. Le fossé T.4 m.172, situé dans la partie centrale du marais, a livré des graines d'orge (hordeum vulgare). Par ailleurs des pollens de céréales assez abondants associés à des plantes rudérales (Chénopodiacées, armoise) ont été retrouvés dans un échantillon prélevé dans une couche antérieure au milieu du $\mathrm{I}^{\mathrm{er}}$ s. av. J.-C. située sur le bord du Girondan (Berger et al., 2003).

La faune retrouvée dans les niveaux laténiens est trop peu abondante pour constituer un échantillon réellement représentatif (40 restes dont 24 identifiables, voir tabl. I). Malgré tout, on constate l'importance quantitative des restes de porcins ( $70 \%$ des individus) et la prépondérance de l'apport de viande bovine (64\% du poids de viande). Par ces caractéristiques, ce lot peut être rapproché, avec précaution, de séries plus abondantes issues de sites fouillés dans la région lyonnaise et datées de La Tène finale (Forest, 1998). Aucune donnée n'est actuellement disponible sur les productions artisanales.

\section{LA VILLA PRÉCOCE (30 AV. J.-C./15 APR. J.-C.)}

Vers 40 ou 30 av. J.-C., la ferme est détruite et laisse place à une première villa qui va différer de la faune tant par son plan que par les matériaux employés pour l'édifier.

\section{EMPRISE ET CARACTÉRISATION DE LA VILLA}

Les restes de sept bâtiments et des aires d'activité ont été retrouvés sur environ 2 ha (fig. 11). La villa est implantée sur la levée alluviale jusqu'au bord de la vallée du Girondan à l'ouest, et vers le canal de drainage du marais au nord, son extension vers le sud et l'est n'étant pas encore reconnue. Elle présente un plan éparpillé sans ordonnance ni orientation rigoureuse. En l'absence de clôture et de voie d'accès, les seuls aménagements extérieurs repérés sont des sols de cour stabilisés par des lits de graviers ou de petits galets. Tout au plus peut-on identifier une concentration de constructions soignées sur la partie la plus élevée de la clairière (sols à 202,50 m NGF) tandis qu'à l'ouest, sur le rebord de la levée alluviale et, en contrebas, dans le lit majeur du Girondan, se développent plutôt des zones d'activités.

En s'installant sur la partie haute, la plus sèche, les occupants n'ont pourtant pas fait l'économie d'un réseau d'assainissement. De nombreuses tranchées drainantes remplies de galets ont été installées en même temps que les bâtiments. Mais des drains peuvent présenter aussi une morphologie très différente (coffre de pierres plates, imbrices emboîtées...) attestant des tentatives d'assainissement réitérées. Dans la partie basse, l'assèchement est simplement assuré par un système de petits fossés convergeant vers les zones déprimées de la clairière. Si l'on a retrouvé plusieurs conduites d'évacuation des eaux usées, on ne dispose pour cet état d'aucune information sur l'origine et le système de répartition de l'eau propre.

\section{LA PARTIE SOMMITALE}

Les parcelles situées au sud de l'église actuelle (AI181 et AI2) ont livré à ce jour les restes de trois bâtiments (fig. 11).

\section{Mode de construction}

La villa fut édifiée sans remblai d'installation préalable. Les constructeurs utilisèrent pour leurs maçonneries en opus incertum des moellons grossiers à une seule face dressée issus de carrières de calcaire bathonien du plateau de l'Isle-Crémieu. Les murs, larges de 1,5 pied, reposent sur des fondations en galets du Rhône jetés à sec dans des tranchées larges de 0,70 m. En complément de la pierre, la terre cuite architecturale pour la toiture mais aussi pour les encadrements de porte et pour des fûts de colonne a été largement employée. Les tegulae furent produites - au moins en partie - dans un atelier dépendant de la villa (Pinet, 1972).

Il n'a pas été possible de distinguer un schéma métrologique comme cela a pu être le cas, par exemple, dans la villa contemporaine de Richebourg dans les Yvelines (Barat, 1999). 


\section{Le bâtiment IA}

Seule l'extrémité méridionale de cette construction a pu être fouillée (fig. 11). Il semble qu'il s'agisse du bâtiment résidentiel.

Il présente un plan avec galerie de façade P6, orientée au sud-ouest, large de 3,80 m (13 pieds). Celle-ci dessert la pièce centrale $\mathrm{P} 1$ dont seule la profondeur est connue ( $8,50 \mathrm{~m}$ ou 25 pieds). Laile méridionale du bâtiment est divisée en trois pièces longées par un couloir P2, large de 1,20 m. L'espace P3 largement ouvert sur la galerie formait peut-être un pavillon d'angle non saillant. La jouxtant au nord, P4 est la seule pièce à être dotée initialement d'un sol en mortier de chaux.

Cette construction a connu des modifications dont la plus notable fut le redécoupage de la pièce 1 . Au moins deux petites pièces ont été installées dans la partie sud de cet espace. Dans la pièce la, dont seul est connu l'angle sud-ouest, se trouvait un foyer aménagé doté d'une sole en dallettes calcaire. L'espace de 2,60 m de côté (7,5 pieds) créé à l'angle sud-ouest de la pièce 1 par l'aménagement de Pla fut aussi ultérieurement cloisonné par un muret de pierre sèche $(\mathrm{P} 1 \mathrm{~b})$.

P4 est la seule pièce ayant connu une réfection de sol avec la pose, directement sur le niveau de terre battue initial, d'une chape de mortier de tuileau granuleux. Ces évolutions ne peuvent pas être situées dans le temps par rapport à l'état initial.

\section{Le bâtiment IB}

Intégralement fouillé, il est possible de le reconstituer dans son intégralité par symétrie. Cet édifice, situé à $6 \mathrm{~m}$ au sud du bâtiment IA, présente une orientation en différant légèrement $\left(18^{\circ} \mathrm{O}\right)$ mais partage avec lui de nombreux points communs. D'une forme carrée de $15,80 \mathrm{~m}$ de côté hors tout (soit un espace intérieur de 50 pieds de côté), il s'ouvre vers l'ouest par une galerie de façade de même profondeur que IA. De même, ses nefs latérales présentent la même largeur (14 pieds) que l'aile méridionale du bâtiment IA et plusieurs pièces ont des dimensions identiques. Mieux conservé que IA, il a gardé ses bases de portes ce qui permet de restituer une distribution depuis l'espace central P2.

La stratigraphie intérieure se répète dans toutes les pièces. Le niveau de travail est recouvert d'un remblai limoneux homogène épais d'une douzaine de centimètres. Sur ce remblai, on trouve un radier d'une épaisseur de pierres posées de chant à sec. Le sol, une chape de béton de chaux, n'a été conservé que ponctuellement, dans la galerie P1.

La fonction de ce bâtiment reste inconnue faute de matériel ou d'installation représentative. Plusieurs indices trouvés en limite de fouille indiquent qu'un corps de bâtiment de taille inconnue se développe en retour au sudouest de IB dans les parcelles AI2 et AI3.

\section{Le bâtiment IC}

Une troisième construction orientée à $9^{\circ} \mathrm{O}$ a été repérée par sondage dans la parcelle AI2, à l'ouest des précédents. Des différences dans le gabarit des matériaux laissent supposer qu'il ait été érigé postérieurement aux autres. Sa stratigraphie qui présente une succession d'une dizaine de niveaux de terre sur une épaisseur de près de $50 \mathrm{~cm} \mathrm{ne}$ permet pourtant pas de distinguer une nette différence de chronologie. Son occupation qui débute au début du dernier tiers du ${ }^{\mathrm{er}} \mathrm{s}$. av. J.-C. n'apparaît pas plus longue et s'achève, comme IA, par un incendie vers 15 apr. J.-C.

\section{LES ZONES D'ACTIVITÉ DE LA VILLA}

D'autres installations liées aux activités agricoles ou artisanales sont disséminées au nord et à l'ouest de la partie sommitale et dans le lit majeur de la rivière (fig. 11). Au nord de la butte alluviale, distant d'une cinquantaine de mètres et à plus de $1 \mathrm{~m}$ en contrebas, les sondages ont fourni une vision lacunaire de la pars rustica. Les fragments de quatre bâtiments à fondations de galets et à sols en terre battue, très similaires aux édifices précédents, ont été retrouvés dans le secteur fouillé. Leurs plans et leurs fonctions précises ne sont pas connus. Associées à ces constructions, on trouve plusieurs cabanes semi-enterrées quadrangulaires dont une seule, de $3 \mathrm{~m}$ de côté, a été fouillée. Le sol en gravier y est aménagé à l'intérieur d'un creusement carré aux angles arrondis et aux parois verticales profondes de $30 \mathrm{~cm}$. La couverture de tuiles repose sur deux séries de trois poteaux calés contre les parois est et ouest. Un muret en pierre sèche non fondé, bordant la paroi sud, en était la seule superstructure conservée. Faute de matériel représentatif, sa fonction reste inconnue.

Plus à l'ouest, en bordure de la plaine d'inondation du Girondan, les sondages ont mis en évidence une cabane et des trous de poteaux associés à des dépotoirs contenant des déchets de forge et de tabletterie. L'étendue limitée des sondages par rapport à la surface concernée (environ $7 \%$ ) 


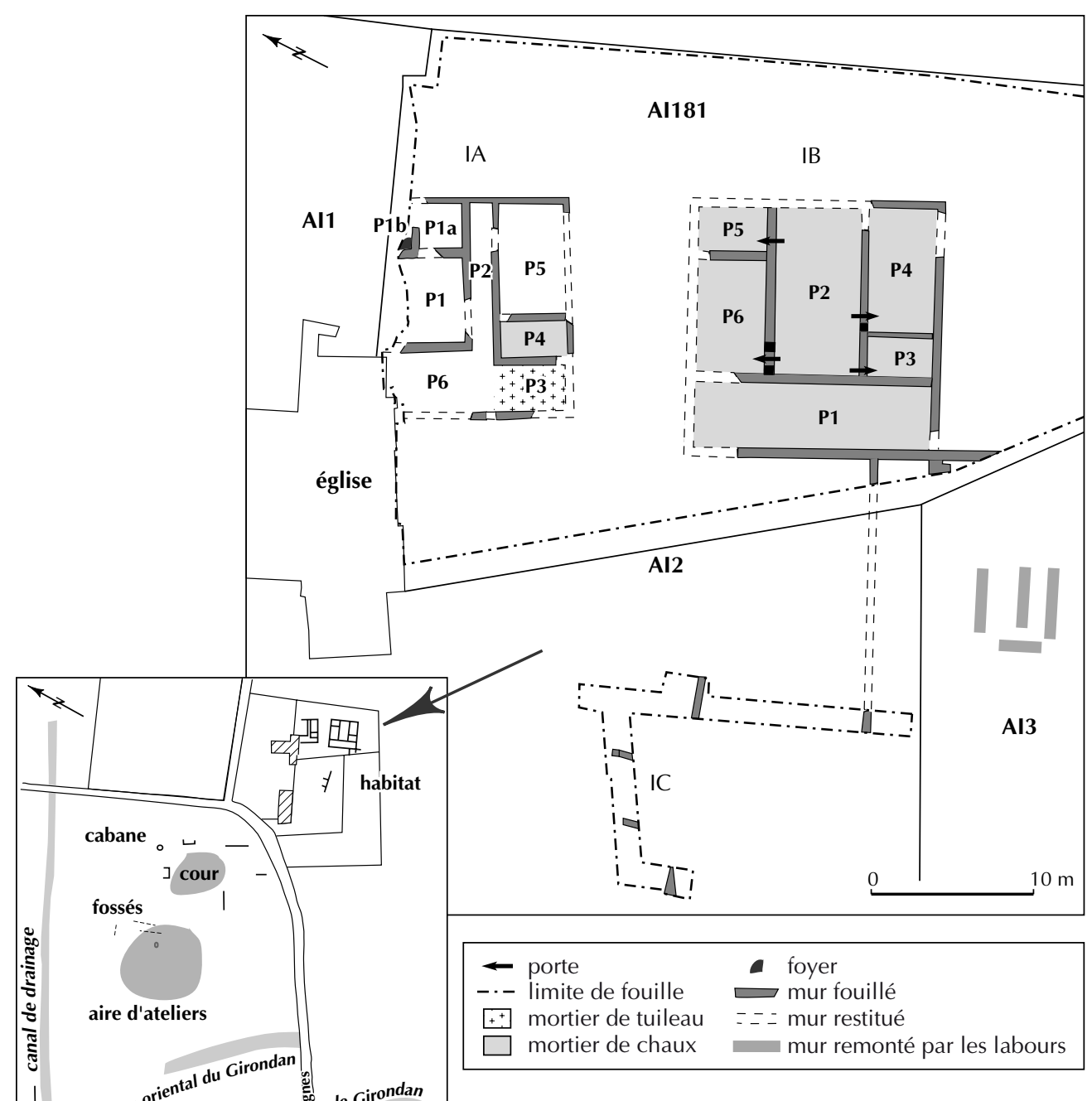

ne permet pas de se prononcer sur le détail de l'organisation de cet espace qui s'étend sur plus de $1200 \mathrm{~m}^{2}$.

\section{ENTRE LA VILLA ET SON DOMAINE:}

\section{L'AMÉNAGEMENT DU LIT MAJEUR DE LA RIVIÈRE}

Le Girondan, dont le régime se modifie à plusieurs reprises dans la seconde moitié du $\mathrm{I}^{\mathrm{er}} \mathrm{s}$. av. J.-C. connaît, d'importants travaux de régulation visant au moins à conquérir son lit majeur. Son bras oriental s'encaisse tout d'abord en un chenal peu profond mais large d'une dizaine de mètres dans les alluvions grossières accumulées au cours des décennies précédentes (pl. VIII hors texte). Ce bras se colmate très rapidement sous l'effet, d'une part, d'une accumulation naturelle et progressive de sédiments fins limono-argileux et, d'autre part, d'un apport volontaire de matériaux grossiers constitué notamment de débris de constructions. À proximité des ateliers métallurgiques, le chenal du Girondan a servi de dépotoir et est partiellement remblayé par d'épaisses couches de charbons de bois et de scories mêlées à des tegulae.

Ces apports cumulés entraînent l'atterrissement progressif de ce bras oriental et le retour de la rivière dans son lit initial. Entre la fin du $\mathrm{I}^{\mathrm{er}}$ s. av. J.-C. et la seconde moitié du $\mathrm{I}^{\mathrm{er}}$ s. apr. J.-C., le régime du Girondan est caractérisé par un débit important déposant une charge de fond 
grossière qui rehausse le fond de son lit de près de $60 \mathrm{~cm}$ (pl. VIII hors texte). On ignore comment était aménagé le passage de la rivière, mais il est clair que les deux rives ont été drainées et fréquentées. De larges fossés de drainage parallèles à la rivière ont alors été creusés à une trentaine de mètres de celle-ci. Les pollens recueillis dans les différentes tranchées fournissent des images contradictoires de l'utilisation des abords de la rivière. Cette période voit une disparition temporaire de la ripisylve. Le lit majeur devient une prairie humide (présence de Cichoriées, de Poacées dans la tranchée 25). Mais la présence de pollens de céréales associée à une diminution notable des Poacées par rapport aux niveaux antérieurs prélevés $50 \mathrm{~m}$ plus au nord des précédents (T.45) suggère qu'une partie de la plaine du Girondan fût mise en culture après drainage. L'utilisation agricole des abords de la rivière pourrait avoir essentiellement concerné la rive gauche. La rive droite, située à proximité immédiate des installations de production de la villa, aurait plutôt été utilisée pour des activités artisanales ou comme espace de divagation du petit cheptel. À défaut d'identifier des installations caractéristiques, il faut signaler la présence de pollens de chanvre dans un prélèvement à peu de distance de la rivière dans la tranchée 45 . Le très fort pourcentage de ce taxon $(41 \%)$ et sa présence très localisée suggèrent la proximité d'activités de rouissage de cette plante textile plutôt qu'une pollinisation depuis des champs voisins (fig. 9). Ces utilisations polluantes rendent donc le Girondan impropre à la fourniture d'eau potable.

\section{LE DOMAINE : EMPRISE ET MISE EN VALEUR}

La détermination du domaine utile de la villa au $\mathrm{I}^{\mathrm{er}}$ s. av. J.-C. se heurte à l'absence d'informations sur l'utilisation des terrains secs. La prospection n'a guère livré de gisements de cette période sur ce type de sols. Seul un atelier de tuiliers au lieu-dit le Terreau a, dès la seconde moitié du I ${ }^{\text {er }}$ s. av. J.-C., exploité des poches argileuses dans le nord de la commune (Pinet, 1972) (fig. 12). Si la photointerprétation et des opérations d'archéologie préventive ont, à plusieurs reprises, mis en évidence des fossés galloromains, ceux-ci n'ont pu être datés plus finement. Il faut donc se résoudre à circonscrire le périmètre d'influence possible de la villa en dressant la cartographie des sites contemporains importants localisés à Leyrieu, à l'est, et à Tignieu-Jameyzieu et Villemoirieu, à l'ouest.

Il est, en revanche, possible d'affirmer que les zones humides, plaine d'inondation du Girondan ou marais du Grand-Plan, ont été mises en valeur pour assurer la plus

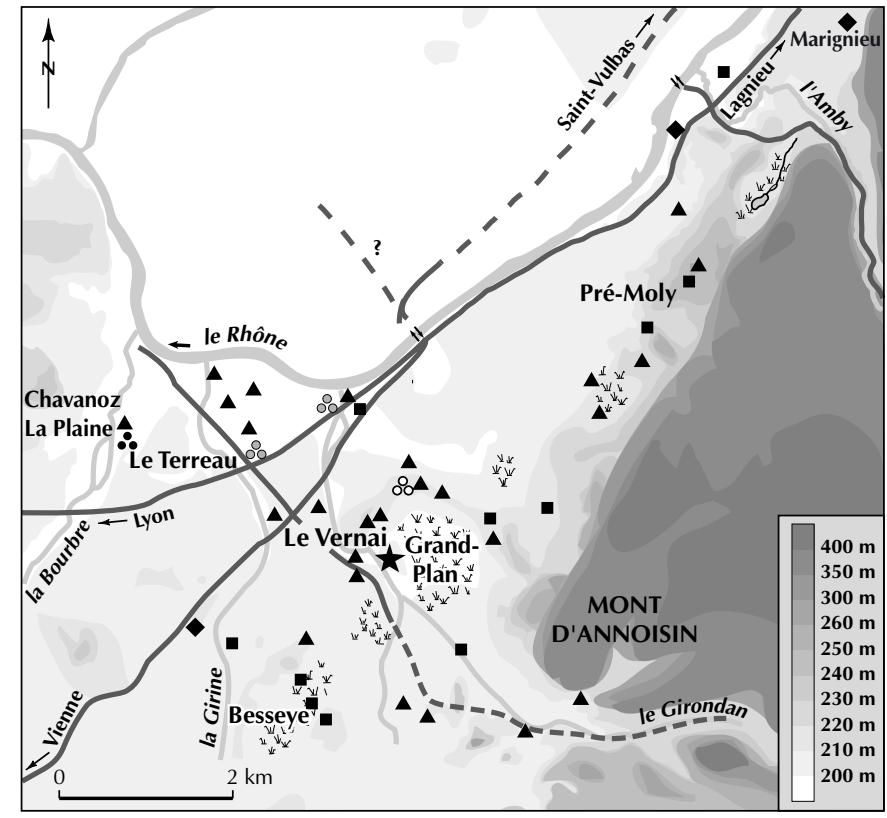

- villa $\quad$ ferme dépendance $\therefore$ atelier de tuilier

$\therefore$ tuilerie ou atelier de potier $\therefore$ atelier de métallurgie

Fig. 12 - Carte des abords du site du Vernai aux $I^{e r}-I I^{e} s$. (DAO R. Royet, SRA Rhône-Alpes).

grosse part des revenus agricoles de la villa. Le marais du Grand-Plan a fait alors l'objet de travaux de bonification de grande ampleur. Les tranchées ouvertes dans l'extrémité nord-ouest de la dépression marécageuse ont permis de confirmer l'existence des réseaux parcellaires initialement détectés par G. Chouquer (inédit). Le paléosol déjà partiellement cultivé lors de l'époque précédente est alors drainé par un réseau complexe sur toute la partie sondée du marais. Deux orientations principales prédominent (fig. 13 et 14). La plus importante, calée selon les points cardinaux, organise le nord et toute la moitié orientale du marais. La seconde, plus limitée, se raccorde sur la première et structure à l'ouest du marais les terrains les plus proches de la villa dont elle reprend l'orientation dominante (autour de $\left.26^{\circ} \mathrm{O}\right)$.

Les principaux axes de ces réseaux sont constitués de fossés géminés encadrant vraisemblablement un chemin de terre large, selon les cas, de $3 \mathrm{~m}$ ou $6 \mathrm{~m}$. L'estimation de l'écartement entre les fossés et leur description restent imprécises, ceux-ci ayant été recoupés obliquement et plusieurs fois curés. Leur profil forme une auge à fond plat et parois évasées, large d'environ 1,20 m à l'ouverture et d'une profondeur variable oscillant entre $0,50 \mathrm{~m}$ et plus de $1 \mathrm{~m}$. 

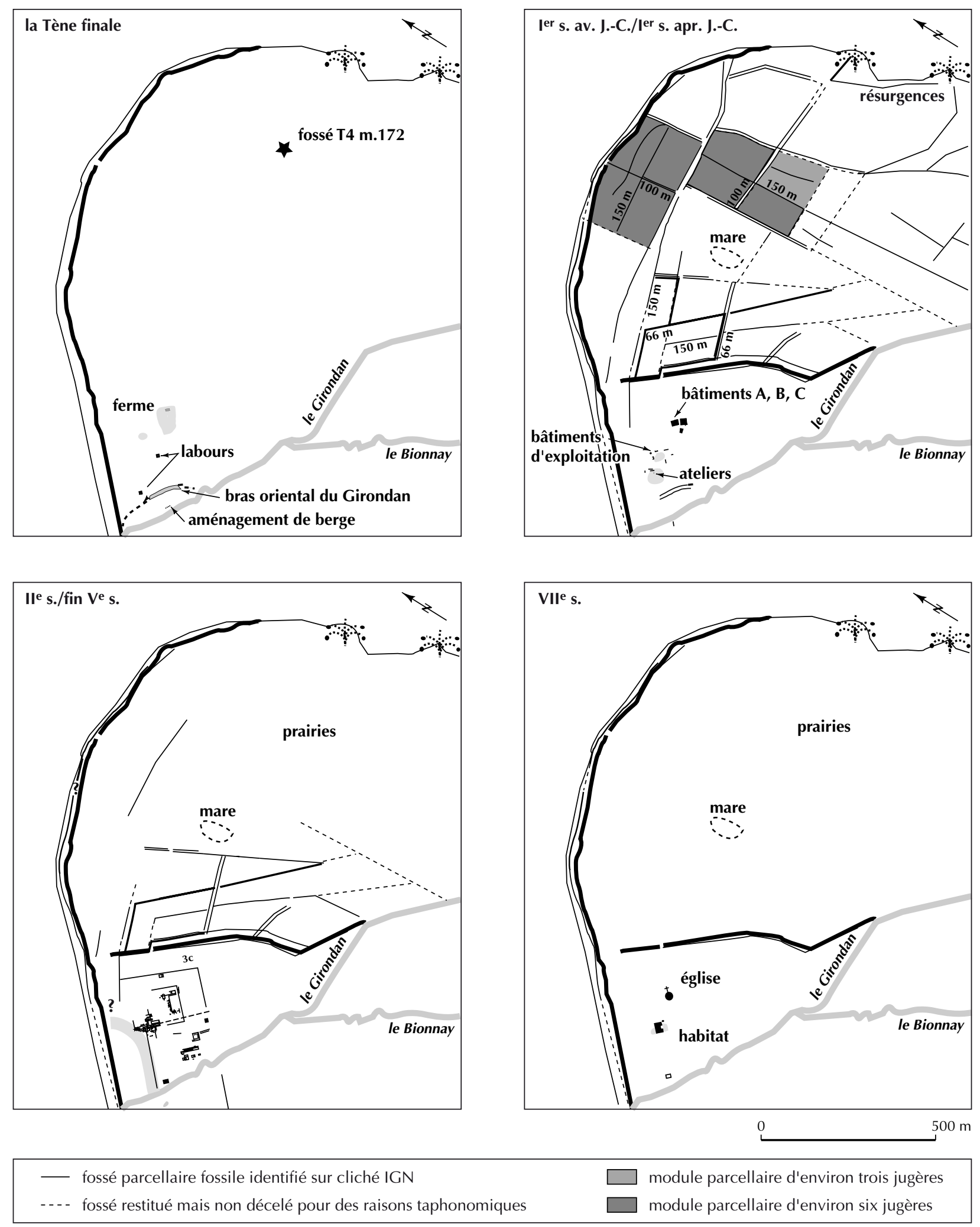

Fig. 13 - Évolution du parcellaire dans le marais du Grand-Plan

(DAO J.-F. Berger et N. Bernigaud, CNRS; R. Royet, SRA Rhône-Alpes). 
Fig. 14 - Les aménagements hydrauliques antiques du marais du Grand-Plan et de la Besseye (DAO N. Bernigaud, CNRS).

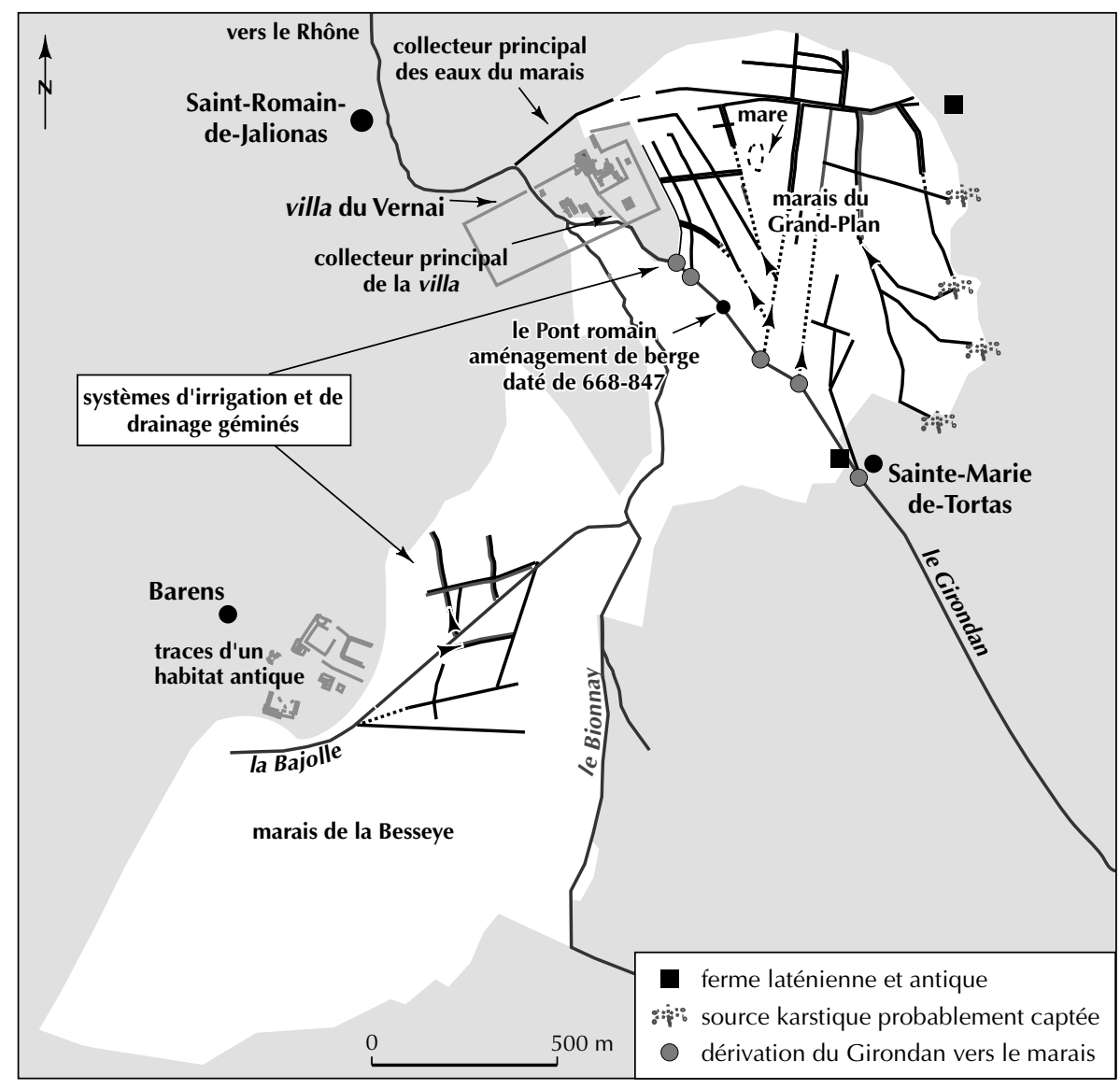

L'ensemble des fossés orientés selon les points cardinaux dessine un quadrillage contrarié par la géométrie de la dépression. De grandes unités rectangulaires de $150 \mathrm{~m}$ x $100 \mathrm{~m}$ peuvent être restituées. Les fossés orientés à $26^{\circ} \mathrm{O}$ délimitent des surfaces souvent trapézoïdales, plus petites et plus allongées, d'environ 150 m x 66 m. Dans tous les cas, la précision de la mesure est insuffisante pour permettre de transcrire ces modules en multiples de la jugère. Plusieurs indices laissent à penser que ces espaces étaient divisés par des haies. Outre la reconnaissance de chablis recoupés dans les tranchées d'évaluation (T.4 m.123), l'étude des charbons prélevés dans les comblements des fossés souligne en effet l'importance d'espèces de lisières (orme, noisetier, rosacées) associées au chêne.

Dans l'espace séparant les deux systèmes parcellaires au centre du marais, une tranchée a recoupé une petite dépression (T.2 m.73 à m.110) située dans un large bras mort du Girondan datant de l'Holocène ancien. Une colonne pollinique y a été prélevée (T.2 m.94,5, voir fig. 20). L'échantillon correspondant au début de l'Antiquité montre, outre la disparition des espèces boisées attestées dans les niveaux inférieurs, la multiplication des herbacées caractérisant les espaces ouverts (Graminées et Cichoriées) et humides (Cypéracées) ainsi que la présence du potamot, indicateur d'eau libre. Il semble donc qu'une mare, précieuse pour l'entretien du bétail, ait occupé la partie la plus déprimée du Grand-Plan (fig. 14). Une partie des terrains humides a dû être utilisée en prairies permanentes ou provisoires en relation avec la rotation des cultures. L'analyse du matériel osseux consommé sur le site montre un élevage sur place centré sur le couple bœuf-porc (tabl. I). Si les porcs peuvent avoir été utilisés à nettoyer les taillis ou les chênaies (qui se raréfient à cette époque) des zones périphériques du domaine, il fallait conserver pour les bovins des surfaces suffisantes de pâturage.

Ailleurs dans le marais, les sédiments colmatant les fossés permettent de préciser leur évolution. Ces apports successifs, entre lesquels s'intercalent des curages, sont notamment constitués de limons fins et de sables associés à des gravillons et des fragments de tuf. Ces alluvions limoneuses, qui possèdent une structure massive à porosité fermée indiquant une hydromorphie presque perma- 
nente, sont morphologiquement proches du paléosol. L'accumulation de ces alluvions dans les fossés semble leur conférer une fonction de drainage prévisible dans un marais. On peut toutefois envisager une ambivalence de ces canaux. La présence de microlits de sables et de graviers, parfois en alternance avec des sédiments fins limonoargileux, évoque en effet la possibilité d'une irrigation. Cette deuxième fonction pourrait expliquer l'organisation de ces réseaux. Dans la moitié occidentale du marais, les lignes directrices d'orientation nord-sud paraissent se prolonger jusqu'à des points d'inflexion du Girondan. Le cours de cette rivière, constitué dans sa traversée du marais d'une série de segments rectilignes, semble avoir été rectifié et, en tout cas, stabilisé. On peut proposer, à titre d'hypothèse, que ces points d'inflexion où aboutissent les réseaux antiques correspondent à un système de dérivation en patte d'oie comparable à un aménagement médiéval ou moderne improprement nommé Pont romain (voir infra, p. 319), mais qui était en fait une prise d'eau sur la rivière pour alimenter un étang (Bernigaud, 2002).

Une seconde alimentation de ces fossés peut être fournie par les résurgences karstiques présentes au pied du versant ouest de la moraine (fig. 14). Plusieurs sources, signalées sur le cadastre napoléonien, ont été équipées de bassins de captage à l'époque moderne, pouvant masquer des aménagements plus anciens. L'une d'elles est directement liée au réseau antique par un fossé dont l'ancienneté est avérée par sondage (T.4 m.383). Pour trois autres, le raccordement avec ce réseau antique est réalisé grâce à des canaux convergeant vers un même fossé abandonné dès la fin du I $^{\text {er }}$ s. apr. J.-C. Cette double alimentation peut se justifier en partie par le tarissement du Girondan constaté lors des périodes de sécheresse.

La mise en valeur du marais s'accompagne donc d'un réaménagement du cours du Girondan dont les conséquences se font sentir dans les alluvionnements étudiés en aval (démantèlement de formations tufacées dont les débris se retrouvent dans la tranchée 56). La rivière possédait donc un rôle d'alimentation dans sa traversée du marais et de drainage en aval de la villa, grâce à un collecteur passant au pied de la butte morainique à quelques mètres au nord de l'actuel canal de drainage.

L'ampleur des travaux de bonification des zones humides reste à évaluer, les tranchées ayant été cantonnées à la rive orientale du Girondan. La photo-interprétation de missions anciennes de l'IGN permet toutefois de repérer des fossés fossilisés dans le marais de la Besseye situé au sud-ouest du marais du Grand-Plan (fig. 14). Ces deux marais sont en fait étroitement liés même s'ils ne constituent pas un géosystème unique. Le marais de la Besseye est alimenté par le Bionnay, qui conflue avec le Girondan à hauteur de la villa. Dans la Besseye, les linéaments fossiles détectés ont été retrouvés de part et d'autre de la Bajolle, un affluent du Bionnay. Comme dans le Grand-Plan, plusieurs fossés géminés espacés de $150 \mathrm{~m}$ forment un réseau quadrillé complété par les fossés rayonnants. Ce dispositif ne peut être confondu avec les tentatives de drainage engagées au cours de la seconde moitié du XIX $^{\mathrm{e}} \mathrm{s}$. et caractérisées par un parcellaire découpé en lanières. Cet ensemble, limité dans l'espace et séparé du réseau du Grand-Plan, pourrait donc lui être contemporain. Il n'est en tout cas pas certain que tous les terrains humides des marais de Saint-Romainde-Jalionas aient été drainés. Ainsi, aucune trace d'aménagement n'a été repérée sur la rive gauche du Girondan et, dans le marais de la Besseye, seule la partie centrale est quadrillée. La permanence de réserves humides, servant entre autres de terrains de chasse, paraît être confirmée par l'analyse de la faune consommée sur le site qui comporte une part non négligeable de gibier d'eau.

Dans l'état actuel de la connaissance des parcellaires fossiles détectés dans les marais de la Besseye et du GrandPlan, on peut restituer un espace humide d'environ 200 ha situé au sud de Saint-Romain-de-Jalionas et divisé en deux parties d'importance comparable. Les parties du marais du Grand-Plan à l'est du Girondan et de la Besseye situées à proximité du hameau de Barens auraient été cultivées alors que le reliquat serait resté en taillis ou en prairies. Il n'est pas certain que la totalité de cet espace humide ait été intégrée au domaine de cette villa. Au moins deux autres sites, localisés à proximité des hameaux actuels de Barens et Sainte-Marie-de-Tortas, étaient implantés sur le rebord des marais. Mais la grande cohérence et la hiérarchisation des fossés du système d'assainissement du marais du Grand-Plan laissent malgré tout supposer une unité de fonctionnement et de propriété. Malgré son origine ancienne et sa longue séquence d'occupation, le gisement de Sainte-Marie-deTortas ne présente pas les caractéristiques permettant de lui accorder le statut de villa. Il semble qu'il s'agisse plutôt d'une ferme dépendant du Vernai.

\section{LES PRODUCTIONS DE LA PREMIÈRE VILLA}

La céréaliculture est un des deux piliers de l'économie agricole de cette exploitation. Des graines de blé amidonnier ont été trouvées lors du tamisage de colmatages de certains fossés du marais (Ploton, 2001). Toutes les colonnes 
polliniques prélevées en bordure du Girondan montrent un accroissement de la fréquence des pollens de céréales à la fin du $\mathrm{I}^{\mathrm{er}}$ s. av. J.-C. (fig. 9). Leur faible potentiel de dispersion induit la proximité immédiate d'une culture, peut-être située dans le lit majeur de la rivière.

Pour cette période il ne s'agit pourtant pas d'une monoculture, la forte probabilité d'une production de chanvre ayant déjà été évoquée. Si la très forte densité de pollens retrouvée dans la tranchée 45 est vraisemblablement révélatrice d'une activité de rouissage (voir supra, p. 298), ce taxon se retrouve en plus faible quantité dans les différentes tranchées ouvertes dans la partie occidentale du site. Comme pour les céréales, cette production paraît avoir été développée dans les terres humides et profondes du lit majeur du Girondan. Elle n'a toutefois certainement pas pu couvrir des surfaces très importantes, le chanvre étant en effet totalement absent dans les colmatages des fossés du marais et la terrasse fluvio-glaciaire, aux sols arides et maigres, n'étant pas propice à ce type de plantation.

Parallèlement à la culture, l'élevage a certainement constitué une source de revenus notable. L'échantillon de 570 restes identifiés provenant de 81 US montre une consommation privilégiant porcins (276 restes) et bovins (60\% du poids de viande) de petits gabarits. Le déficit de certaines pièces, l'absence de bovins juvéniles et de grande taille plaident pour une production sur place en partie exportée.

À côté des espèces domestiques traditionnelles, les niveaux de cette villa recèlent une part non négligeable d'animaux sauvages notamment issus d'un biotope humide (tabl. I). On note surtout la présence de petit gibier, notamment d'oiseaux (bécasse, sarcelle, colvert) mais aussi de lièvres. En revanche, le grand gibier est absent, les restes de cerf correspondant uniquement à des ramures, vraisemblablement collectées après leur chute. L'importance alimentaire de cette chasse ne doit toutefois pas être surestimée, le poids en viande en découlant apparaissant dérisoire au regard de ce que procure l'élevage.

L'artisanat tient une place difficilement quantifiable dans l'économie de cette villa. Si le traitement du chanvre est attesté, on ne trouve aucun témoin d'une activité de tissage. En revanche, la métallurgie du fer est certaine. Le lit du Girondan ainsi que plusieurs fosses-dépotoirs ont livré d'importantes quantités de scories, reliquats de forgeage, associées à des restes osseux, cornillons sciés ou baguettes calibrées, préparés pour un travail de tabletterie. L'importance de l'aire de répartition des installations et des concentrations de scories (plus de $1200 \mathrm{~m}^{2}$ ) conduit toutefois à penser que cette activité dépassait la simple couverture des besoins domestiques.

À l'extérieur de la villa, il n'est pas exclu que des ateliers aient été installés très tôt, en particulier le long des voies de communication. Même si la tuilerie du Terreau fut créée initialement pour satisfaire aux besoins des bâtisseurs de la villa, sa production, qui se prolonge au moins jusqu'à l'époque flavienne, fut ensuite certainement tournée vers le commerce.

\section{LA GRANDE VILLA (I ${ }^{\mathrm{er}}-\mathrm{II}^{\mathrm{e}} \mathrm{S}$. APR. J.-C.)}

La villa est intégralement détruite vers 15 apr. J.-C., peut-être par un incendie. À sa place, un nouvel établissement d'une dimension inhabituelle pour la région est reconstruit (fig. 5 et 6 ).

\section{L'EMPRISE}

Cette seconde villa couvre près de 13 ha circonscrits au nord par le collecteur du marais et, sur les trois autres côtés, par une enceinte dépourvue de vocation défensive. Le Girondan est alors intégré dans la villa qu'il divise en deux parties à peu près égales.

Occupant le sommet de la butte alluviale, le secteur résidentiel est initialement entouré par un mur d'enclos de $133 \mathrm{~m}$ x $78 \mathrm{~m}$. On ne connaît pratiquement rien des espaces situés à l'est et au sud. À l'ouest, les terrains les plus proches du canal de drainage sont abandonnés, le mur d'enclos marquant la limite $85 \mathrm{~m}$ plus au sud. Les installations économiques repérées se situent sur la rive droite du Girondan, au sud-ouest de la partie résidentielle.

Une voie large de $6 \mathrm{~m}$ crée au début du I ${ }^{\mathrm{er}} \mathrm{s}$. apr. J.-C. bordait la villa au nord. Ce diverticule rejoignait les routes aboutissant au gué de Saint-Oyand et la voie reliant Lyon à Aoste.

Les incessants travaux que connaîtra le site jusqu'à la fin de l'Antiquité ne remettront pas en cause ce plan-masse. On ne peut actuellement pas dresser un panorama de ces transformations qui ne sont, souvent, que locales. Seul l'état initial est partout présent et peut être synthétisé.

\section{LA VILLA AUX I ${ }^{\mathrm{er}}$ ET II ${ }^{\mathrm{e}} \mathrm{S}$.}

\section{La partie résidentielle}

Seules ses extrémités nord-ouest et sud-est ont été partiellement fouillées. La partie centrale reste enfouie sous l'église et, surtout, sous le cimetière. 
L'édification de ce vaste complexe va donner lieu à de gros travaux de viabilisation.

La construction et l'assainissement du site au début du $I^{\text {er }} \boldsymbol{s}$.

De gros moyens vont être mis en œuvre pour endiguer les effets d'une humidité excessive. La stabilité des maçonneries est assurée par des fondations coulées, profondes de $0,80 \mathrm{~m}$ et larges de $0,60 \mathrm{~m}$. Les sols sont installés sur une hauteur moyenne de $45 \mathrm{~cm}$ de remblais limoneux ou issus de la démolition de l'état précédent.

La mise hors d'eau des nouveaux bâtiments s'accompagne de la création d'un réseau de drains et de conduites d'évacuation des eaux usées dès les premières décennies du $\mathrm{I}^{\mathrm{er}}$ s. apr. J.-C. Le collecteur qui en constitue la pièce maîtresse se présente comme un conduit maçonné dont la section interne est de $0,45 \mathrm{~m}$ x $1,45 \mathrm{~m}$ installé dans une tranchée large de $3 \mathrm{~m}$ et profonde de $2,50 \mathrm{~m}$ au point le plus haut du site (fond de la cunette à la cote 199,80 m NGF à l'extrémité sud de la parcelle AH30). Initialement couvert d'une voûte maçonnée, il est, à la fin du $\mathrm{II}^{\mathrm{e}}$ s. ou au début du $\mathrm{III}^{\mathrm{e}} \mathrm{s}$., entièrement restauré et fermé par des dalles posées à sec. Son tracé, à peu près rectiligne, a été repéré depuis l'entrée nord de la villa pratiquement dans toute la traversée de la parcelle AH30 (fig. 17) et, au-delà, sur près de $300 \mathrm{~m}$ grâce à une anomalie visible sur les photos aériennes. L'eau était ainsi évacuée vers le canal de drainage le plus occidental du marais et se jetait dans le Girondan en amont de la villa.

\section{L'alimentation en eau}

Le Girondan dont le fond est $3,50 \mathrm{~m}$ plus bas que certains bassins et qui a servi de dépotoir à partir du milieu du $\mathrm{I}^{\mathrm{er}} \mathrm{s}$. ne pouvait pas procurer l'eau propre au site. Les nombreuses installations hydrauliques ne pouvant être alimentées par des citernes, il est vraisemblable que la villa ait contrôlé une partie du piémont du plateau de l'IsleCrémieu où se concentrent les sources. Les plus importantes et les plus proches se situent sur la commune de Leyrieu, au lieu-dit Pré-Moly, près du seul site précoce dont l'évolution sera bloquée au I ${ }^{\mathrm{er}} \mathrm{s}$. Ces sources peuvent être reliées au site par un tracé théorique permettant à une conduite contournant la Butte-des-Vignes d'accéder au nord de la villa. Une autre possibilité d'alimentation est offerte par la découverte récente d'un tronçon d'aqueduc sur le sommet du plateau de la commune de Villemoirieu. Si cette conduite devait être rattachée au site, il faudrait alors étendre son domaine au sud-est sur $5 \mathrm{~km}$.

\section{L'extrémité méridionale}

Les fouilles de 1985 et le relevé des traces de sécheresse dans la végétation ont permis de mieux connaître l'extrémité sud du secteur résidentiel sur les parcelles AI181 et AI2. Ce corps principal IIA présente une façade d'au moins $46 \mathrm{~m}$, bordée par un portique large de 2,80 $\mathrm{m}$ donnant sur une cour profonde de $27 \mathrm{~m}$. Un pavillon (IIB) délimite l'espace à l'est alors qu'à l'ouest les fouilles n'ont pas localisé les constructions initiales (fig. 15). Du corps principal, seule a été fouillée la partie orientale.

À l'époque tibérienne, le bâtiment IIA ne paraît pas dépasser la largeur de 11,30 m, son extrémité ne comportant que deux pièces, A1 et A2, desservies par un couloir nordsud (PA3). L'organisation du reste du bâtiment (A5 à A10) demeure hypothétique faute de fouille.

Les trois pièces fouillées présentaient un sol en terre battue à la cote 202,60 m NGF. Seul le portique A11, qui doublait la façade, était doté d'un sol en mortier de chaux à la même altitude.

La fonction des cinq pièces du pavillon oriental, à la stratigraphie totalement arasée, demeure inconnue. Des niveaux extérieurs, seul subsiste un sol en terre battue sur un remblai à l'est du bâtiment IIA.

À la fin du $\mathrm{I}^{\mathrm{er}}$ s., le corps principal va s'agrandir vers le nord et être remanié alors que le pavillon IIB semble se figer. À la place de la pièce A2, qui est rasée, est construit un nouvel espace, A12, de $39 \mathrm{~m}^{2}$, équipé d'un chauffage par hypocauste dont le praefurnium était placé face à l'angle sud-ouest (fig. 15, phase 3). Enfin, des murs formant angle droit, V396, dessinent au-delà de la façade une excroissance de $1,80 \mathrm{~m}$.

Dans cette phase, toutes les pièces sont remblayées jusqu'à la cote 203,30 m NGF et leur sol bétonné. Dans le portique est coulé un nouveau sol en mortier de tuileau. À l'ouest, trois murs parallèles peuvent constituer les premiers éléments d'un pavillon occidental IIC.

La construction d'une fontaine à la place de l'aménagement V396 est la dernière modification que connaît ce secteur du bâtiment IIA au II $^{\mathrm{e}} \mathrm{s}$.

\section{Le nord de la zone résidentielle}

\section{L'entrée nord}

$\mathrm{Au}$ nord-ouest de la partie résidentielle, ouvrant sur la voie de desserte du site, a été trouvée la seule entrée connue, une porte charretière numérotée V1499 (fig. 16). 


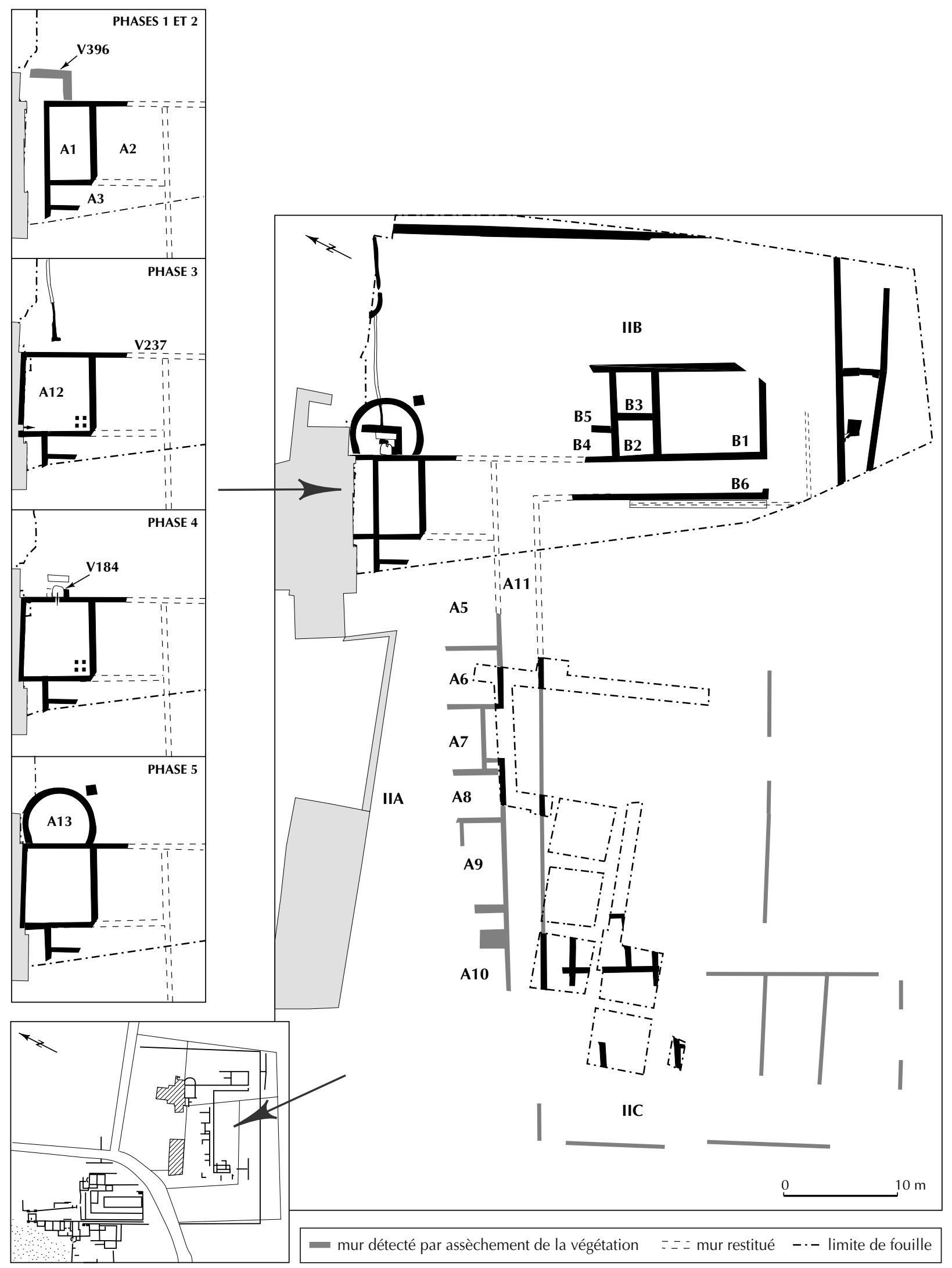

Fig. 15 - Périodisation des vestiges antiques dans les parcelles AI181, AI2 et AI3 du site du Vernai: phase 1, état au début du IPr s. apr. J.-C.; phase 2, modification à la fin du $I^{e r}$ s.; phase 3, état au II ${ }^{e}$ s.; phase 4, état au III $e^{e}$.; phase 5, état au IVe s. (DAO R. Royet, SRA Rhône-Alpes). 

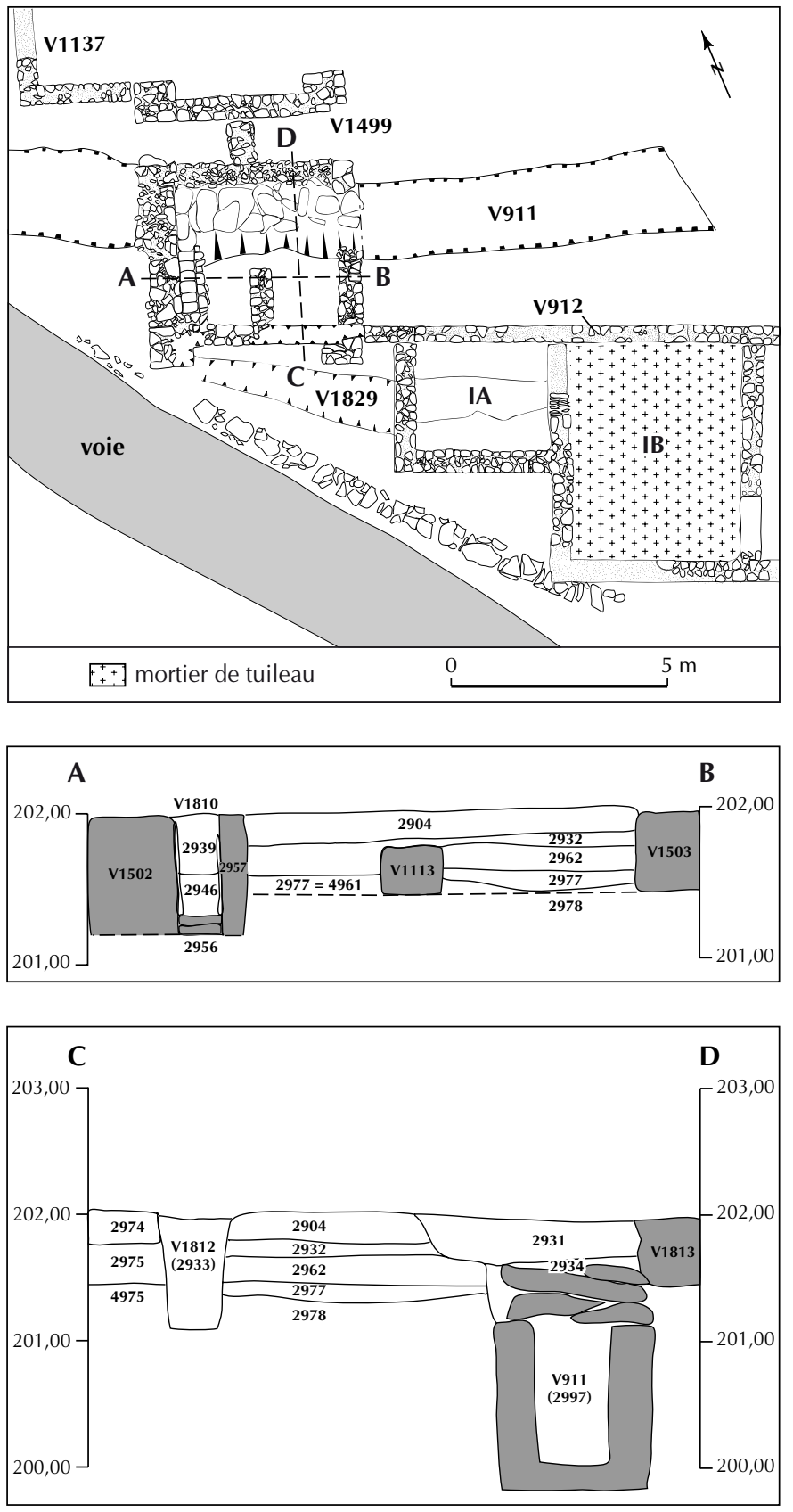

Fig. 16 - Plan et coupes du moulin hydraulique dans la parcelle AH3O du site du Vernai (DAO R. Royet, SRA Rhône-Alpes).

Le passage, large de 4,80 m et profond de $3 \mathrm{~m}$, est encadré par quatre dés carrés reliés par deux murs. Cette porte donne sur une cour profonde de $28 \mathrm{~m}$ et large d'au moins $20 \mathrm{~m}$, fermée par l'ensemble résidentiel au sud et à l'est par un édifice (XII.A) flanquant le mur d'enclos V912.

Sous la porte passe le collecteur principal V911. Cet accès, contemporain au mur d'enclos V912, est condamné dès la seconde moitié du $\mathrm{I}^{\mathrm{er}} \mathrm{s}$. Sur son emplacement et la réemployant partiellement, est édifié un moulin de $5 \mathrm{~m}$ $\mathrm{x}$ 4,10 $\mathrm{m}$ hors tout, aux robustes maçonneries. L'alimentation en eau en était assurée, comme le préconisera Palladius au IV e s., par les eaux usées de bains situés immédiatement au sud (Palladius, De l'agriculture, I, 41). Une tranchée à fond plat peu profonde, V1829, en provenance des bains pourrait être le négatif d'une rampe soutenant cette évacuation. Le coursier était installé à l'intérieur du bâtiment, entre son angle nord-ouest et le collecteur V911 sous-jacent qui faisait office de canal de fuite. Les systèmes d'ancrage qui pouvaient se situer autour de 202,30 m NGF n'ont pas été conservés, mais il est possible de restituer une roue verticale de $2,5 \mathrm{~m}$ de diamètre et de $30 \mathrm{~cm}$ de largeur, comparable à celle du moulin des Mesclans à La Crau (Brun, Borréani, 1998). Le mur V1113 divise l'espace interne en deux parties aux stratigraphies différentes. Au nord, où ne se trouve aucun niveau lié au fonctionnement du bâtiment, pourrait être la chambre d'engrenage, le mur soutenant l'axe de la roue. La chambre de meunerie serait installée dans une superstructure en matériau périssable au sud de V1113. Ce moulin est arasé au plus tard au début $\mathrm{du} \mathrm{II}^{\mathrm{e}} \mathrm{s}$.

\section{Le secteur 7}

Ce carré, d'une trentaine de mètres de côté, situé dans l'angle de la parcelle AH30 et dégagé dans les années 1960-1970, n'a fait l'objet d'une reprise de fouille que sur environ $25 \mathrm{~m}^{2}$ (fig. 17).

Pour les deux premières phases, le plan n'est pas cohérent, mais on peut certifier l'extension, dès l'époque tibérienne, du corps de bâtiment résidentiel à $70 \mathrm{~m}$ au nord des vestiges fouillés en 1985. Le collecteur V911 longe ce complexe.

Une troisième phase de travaux voit le démontage de l'état précédent. La nouvelle façade est décalée de $1 \mathrm{~m}$ à l'est et bordée par un caniveau taillé dans de longs blocs de calcaire bajocien, V1315. Cette phase, datable du $\mathrm{II}^{\mathrm{e}} \mathrm{s}$., pourrait correspondre à l'installation d'un premier état de bains, totalement remaniés ultérieurement. À l'ouest de cette construction est alors délimitée une cour (IIIC), de $31,50 \mathrm{~m}$ x 16,30 m, qui peut être interprétée comme une palestre.

\section{Le bâtiment XIIA}

À l'ouest de la cour, au-delà des murs V912 et V1577 qui délimitaient initialement la villa, cette construction va connaître une évolution en trois phases (fig. 16). 


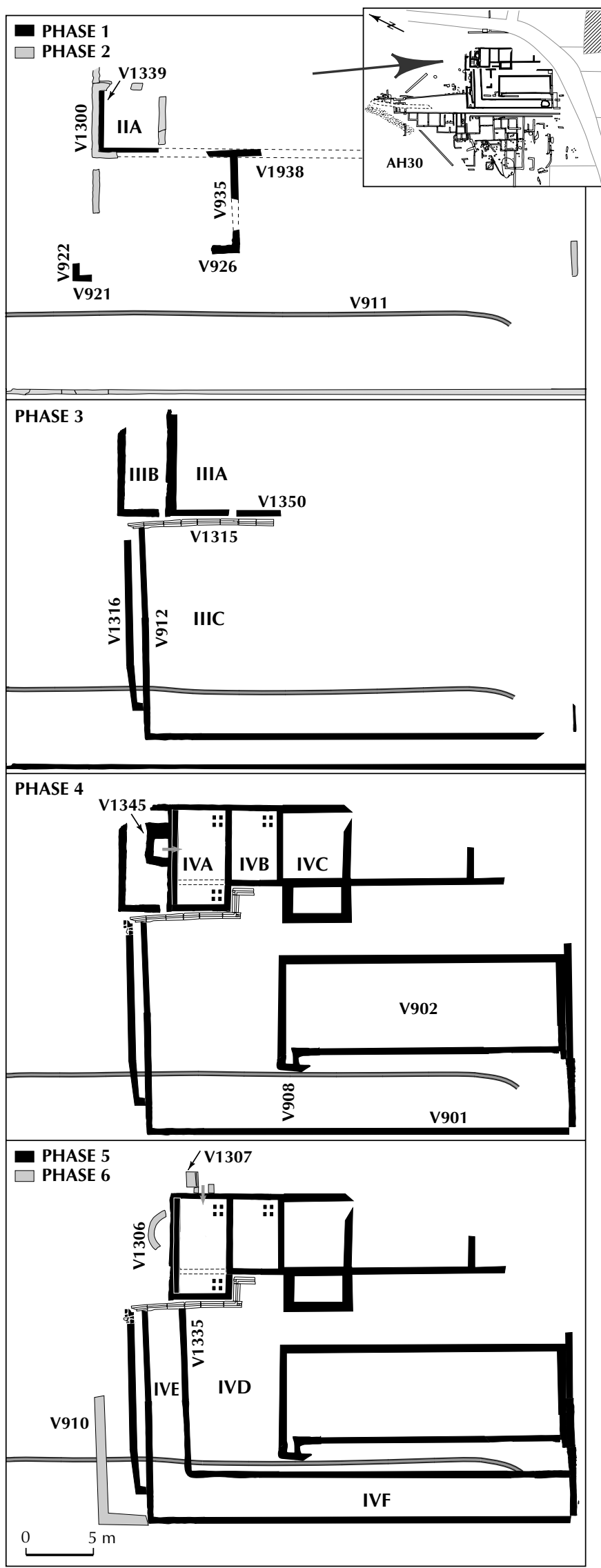

Après l'apport d'un remblai limoneux hétérogène jaune à la cote 201,75 m-201,80 m NGF, on construit au nord de V1577 une enfilade de trois pièces, IB à ID, sans aménagements caractéristiques, prolongée au nord par une pièce IA, deux fois moins profonde. Les murs de cette phase, avec leur élévation en adobe posée sur un soubassement de pierre assisé, large de $54 \mathrm{~cm}$, reposant sur des fondations peu profondes de moellons non triés, présentent une facture originale par rapport aux autres constructions du site. Toutes ces pièces possèdent un sol en terre battue posé sur remblai à la cote 202,00 m NGF. Sous ces sols passe un négatif V1829.

Un agrandissement légèrement ultérieur prolonge à l'ouest le bâtiment d'une pièce (IE).

Ce bâtiment va, au cours du dernier tiers du I ${ }^{\mathrm{er}}$ s. apr. J.-C., être étendu au sud du mur V1577 (fig. 18). Dans la partie fouillée, plusieurs murs sont reconstruits. Leur élévation, large de 0,45 m, présente un appareil assisé de moellons rectangulaires allongés. Des thermes sont alors aménagés. Une chambre de chauffe (IID), de $10 \mathrm{~m}^{2}$, est surcreusée à l'emplacement de l'ancienne pièce ID. Le praefurnium V1555 desservait une baignoire V1569 de $5 \mathrm{~m}^{2}$ et une cella soliaris (IIE) barlongue de $14 \mathrm{~m}^{2}$. À l'extrémité nord de cette dernière pièce, on discerne un négatif V1582, large de 0,40 m, creusé dans le mortier 3677, se prolongeant par une conduite de tuiles plates qui était peut-être une fontaine ou des latrines. Remplaçant IC, une nouvelle pièce IIC occupe l'espace délaissé par le rétrécissement de IID. Les sols des pièces IA, IB, IIC sont relevés à la cote 202,15 m NGF.

Ce secteur n'est qu'un fragment d'un ensemble se développant au sud du mur V1577, à l'intérieur de ce qui paraît être une unité d'habitation indépendante de la partie résidentielle située plus à l'est.

Les niveaux supérieurs sont très dégradés. Aucun sol n'a été conservé et cette phase se distingue essentiellement par des reprises de maçonneries. Il est toutefois possible de distinguer une modification de la fonction des pièces. Le dispositif thermal est démantelé et la chambre de chauffe colmatée par un remblai épais de $30 \mathrm{~cm}$. On remarque dans la pièce IIC un petit drain (V1568). Il est possible que dans cet ultime état les anciennes pièces IID et IIE n’aient plus formé qu'un seul espace. Une cloison de brique

Fig. 17 - Mise en phase des vestiges antiques du secteur 7 dans la parcelle AH3O du site du Vernai: phase 1, état au début $d u I^{e r}$ s. apr. J.-C.; phase 2, modification à la fin du I ${ }^{e r}$ s.; phase 3, état au II ${ }^{e}$ s.; phase 4, état au III ${ }^{e}$.; phase 5, état au IV ${ }^{e}$ s.; phase 6, état au $V^{e} s$. (DAO R. Royet, SRA Rhône-Alpes). 


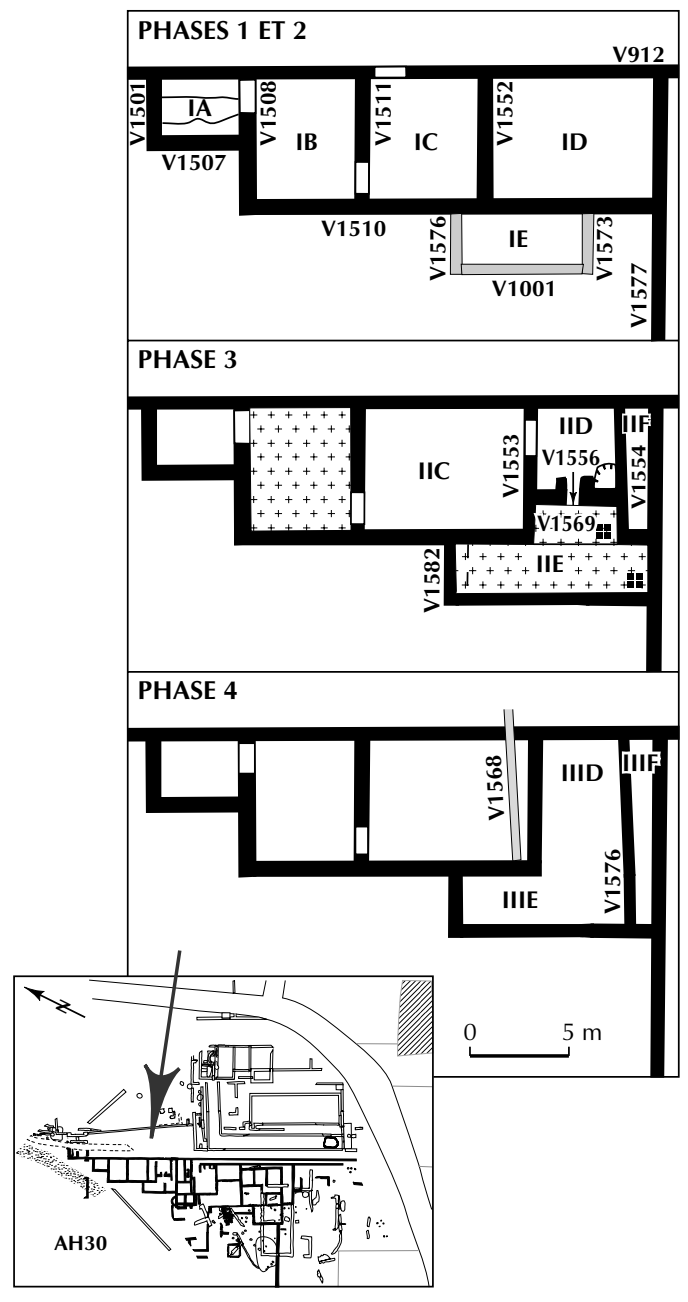

Fig. 18 - Thermes ouest dans la parcelle AH3O du site du Vernai: phase 1, état au début du $I^{e r}$ s. apr. J.-C.; phase 2, modification à la fin du $I^{\text {er }}$ s.; phase 3, état au II $s$; phase 4, état au III $s$. (DAO R. Royet, SRA Rhône-Alpes).

prolongeant V1554 témoigne peut-être d'un escalier installé à l'extrémité du couloir IIIF.

L'absence de matériel interdit de dater cette dernière phase autrement que par chronologie relative.

\section{LA PARS RUSTICA}

Le secteur productif de la villa n'est que très partiellement connu. Il n'est pas organisé comme une cour bordée de part et d'autre de bâtiments mais plutôt comme une juxtaposition d'espaces dévolus à des activités variées: stockage, agriculture, artisanat. La photo-interprétation, pourvoyeuse de la plupart des données, montre à proximité de la rive droite du Girondan, à l'écart de la partie résidentielle, plusieurs lignes d'édifices (secteur 3) orientées comme le reste du site à $27^{\circ} \mathrm{O}$ (fig. 19). Malgré l'ampleur de la villa, il ne semble pas que cette concentration, dont deux bâtiments furent fouillés en 1968-1969, ait regroupé un nombre important de dépendances comme ce fut le cas, par exemple, à Chiragan (Joulin, 1901). L'évaluation conduite dans la parcelle AH30 montre en effet que ces alignements ne se prolongeaient pas au nord-ouest. Au sud de la rivière, les informations demeurent très lacunaires.

\section{Le Girondan et sa plaine d'inondation aux $\mathrm{I}^{\mathrm{er}}$ et $\mathrm{II}^{\mathrm{e}} \mathrm{s}$.}

La majeure partie de nos connaissances sur l'économie de la villa provient de la rivière et de ses alentours (fig. 5 et pl. VIII hors texte).

Alors que le Girondan est intégré dans la villa et que le dérèglement hydrologique déjà constaté lors de la période précédente se poursuit, ses rives ne sont pas stabilisées. Le lit occidental s'évase et, dans le même temps, son fond se rehausse au moins d'une quarantaine de centimètres. L'atterrissement du bras oriental, engagé dès la fin du siècle précédent et accéléré par des remblaiements, s'achève au $\mathrm{I}^{\mathrm{er}} \mathrm{s}$. apr. J.-C. à la suite d'une série de débordements (phase 6). La levée de terre séparant les deux bras est exhaussée pour les mêmes raisons. Les crues qui se prolongent durant la plus importante partie du $\mathrm{I}^{\mathrm{er}}$ s. apr. J.-C. vont ainsi, à plusieurs reprises, inonder une vaste zone dans laquelle est pourtant construit au moins un bâtiment à usage économique (secteur XX, voir infra, p. 309). Il est difficile de préciser la durée de cet épisode, car les colmatages supérieurs ont été érodés par une phase d'incision qui paraît couvrir la plus grande partie du $\mathrm{II}^{\mathrm{e}} \mathrm{s}$.

La plaine d'inondation du Girondan fait au $\mathrm{I}^{\mathrm{er}} \mathrm{s}$. l'objet d'une tentative d'installation. Pour y parvenir les occupants consentent des efforts importants pour limiter les effets d'une humidité excessive. Alors que tous les bâtiments de la villa initiale ont été rasés, une partie, au moins, du système de drainage des zones périphériques a été conservée et entretenue. Le fossé retrouvé dans la tranchée 25, dans la partie sud de la villa, est ainsi curé avant de connaître de nouvelles phases de colmatage (Berger et al., 2003, fig. 6). Un long répit, lié à l'encaissement du Girondan, se prolonge pendant plus d'un siècle. L'arrêt des débordements, associé à un abaissement de la nappe, permet alors le développement d'un paléosol très oxydé qui est localement plus ou moins soigneusement stabilisé par des empierrements (phase $7 \mathrm{a} / \mathrm{d}$ ). 


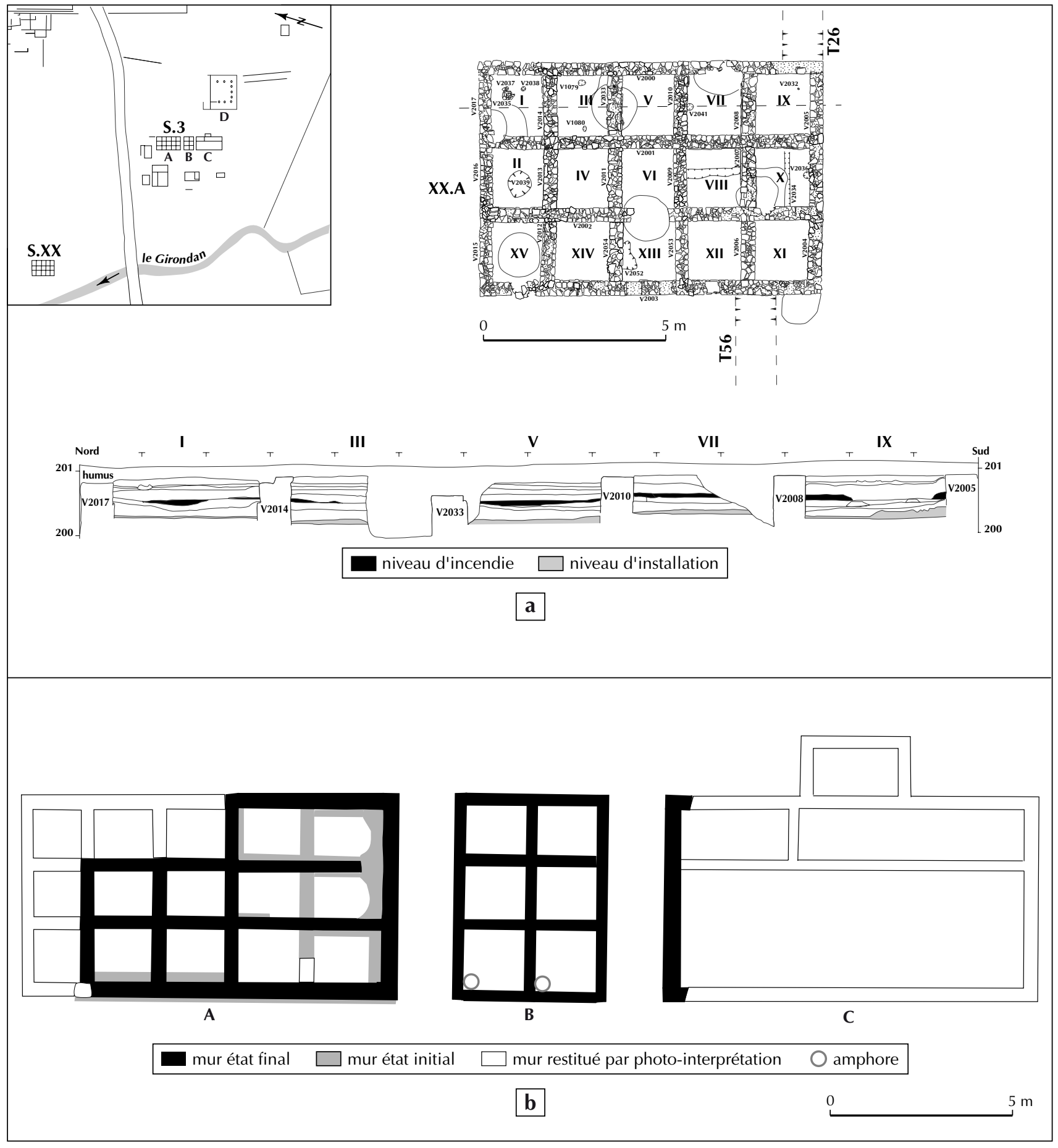

Fig. 19 - Bâtiments de la pars rustica du site du Vernai: a, secteur XX; b, secteur 3 (d'après document R. Pinet). 
La rive gauche de la rivière reste mal connue. L'unique sondage réalisé sur la parcelle AO22, en face de la parcelle AH30, a fourni un niveau de sol stabilisé recouvrant un fossé de drainage contemporain de la villa initiale. La voie de desserte de la villa n'a pu être retrouvée au-delà de sa traversée du Girondan. Un ancien fouilleur déclare avoir décelé un dallage à quelques centimètres sous le fond de la rivière actuelle (communication orale de $\mathrm{R}$. Pinet), mais le fort encaissement du lit antique fait douter que cet aménagement soit gallo-romain. Enfin, la découverte d'une meule de basalte de grand gabarit $(60 \mathrm{~cm}$ de diamètre) rejetée sur la rive de la rivière, à l'extrémité nord du site, laisse supposer l'existence d'un moulin hydraulique. Son fonctionnement aurait été rendu possible par le débit et l'encaissement de la rivière, particulièrement importants à cette période.

$\mathrm{Au}$ cours du Haut-Empire la palynologie restitue un paysage déboisé dans la vallée du Girondan. L'apparition ou le développement de taxons de plantes nitrophiles (Cichoriées, oseille et plantain), y indiquent l'extension des zones de pâturage (fig. 9, échantillons 16 à 23). Le taux réduit de pollens de chanvre dans les échantillons 16 et 17 reflète la culture à proximité du site et non le rouissage pratiqué précédemment.

\section{Le secteur XX}

Les sondages systématiques dans la parcelle AH30 ont amené à la découverte d'un bâtiment isolé (XXA) situé à une dizaine de mètres de la berge (fig. 19 et pl. VIII hors texte).

Cette construction de 9,40 $\mathrm{m} \times 13,75 \mathrm{~m}$ hors tout, qui fut bouleversée par sept chablis lors de la tempête de décembre 1999, est le seul édifice de la pars rustica a avoir été étudié dans le détail. La facture de ses murs et le niveau de travail associé permet de le rattacher à la première phase de la deuxième villa. La couche de démolition présente une texture terreuse fine, pauvre en pierre, qui évoque une élévation à pans de bois et terre. Les négatifs des jambages d'une porte large de $0,80 \mathrm{~m}$ à 1,20 m sont lisibles au milieu de sa façade occidentale. Pour le protéger des débordements de la rivière, ses constructeurs ont imaginé d'en rehausser le sol en créant un vide sanitaire. Pour ce faire, des murs-bahuts forment une grille de quinze compartiments surélevant les solives du plancher de $50 \mathrm{~cm}$ au-dessus du niveau d'établissement.

Les dimensions du bâtiment étant excessives pour une charpente composée de fermes à entrait simple en chêne, il faut supposer une répartition en deux corps. À l'ouest, où la portée habituelle d'une charpente en chêne correspond approximativement à la largeur hors tout de deux compartiments, se situerait le volume principal. La travée occidentale serait un avant-corps. Cette hypothèse implique de restituer deux niveaux au volume principal afin de donner une hauteur suffisante à la travée occidentale. L'étage supérieur serait alors un plancher soutenu par des poteaux installés à l'intersection des refends en fondation. La partie centrale du bâtiment correspondant aux compartiments VI et XIII (qui n'ont pas été fouillés), trop étroite pour servir d'aire de travail, serait la surface de distribution et d'accès à l'étage.

Le dispositif de fondations en grille pouvant supporter de lourdes charges permettrait que cette construction soit un petit horreum avec un espace utile de $180 \mathrm{~m}^{2}$ réparti sur deux niveaux. Toutefois, ce procédé d'assainissement se retrouve dans d'autres bâtiments situés dans les parties humides de la clairière (secteur 3 ) et il n'existe aucune certitude sur sa fonction.

\section{Le sondage 3 au Haut-Empire}

Une dizaine d'autres bâtiments (secteur 3) ont été repérés à une centaine de mètres à l'est du secteur XX. Au moins deux d'entre eux, fouillés en 1968, présentaient un plan comparable et, bien qu'ils soient hors d'atteinte des inondations, le même dispositif de vide sanitaire (fig. 19).

On peut reconstituer pour le bâtiment A un état initial formant un quadrilatère d'environ $15 \mathrm{~m} \mathrm{x} \mathrm{8,30} \mathrm{m,} \mathrm{bâti} \mathrm{en}$ même temps que le bâtiment XXA. La façade ouest comportait deux surépaisseurs en cul-de-four très rubéfiées. Des fragments de parois de fours ont été recueillis, mais l'activité qui leur était liée n'a pu être identifiée. Dans une deuxième phase non datée, il semble partiellement reconstruit.

Distant de $1 \mathrm{~m}$ du précédent et aligné sur lui, le bâtiment $\mathrm{B}$ en reprend la même largeur. Pourtant la facture des murs, plus étroits $(0,35 \mathrm{~m})$ et construits avec des moellons plus réguliers, signale une construction plus récente. Ces deux édifices présentaient des élévations à pans de bois reposant sur des dés de calcaire.

L'alignement décrit se prolonge au sud avec le bâtiment $\mathrm{C}$ dont seul le mur nord a été dégagé. La photo-interprétation permet de lui attribuer une taille identique au bâtiment A avec en plus un porche en saillie au milieu de sa façade orientale. Son organisation interne n'est que partiellement connue et il est possible que le sol repose, ici encore, sur un vide sanitaire. 
À l'ouest de la précédente, la photo-interprétation fournit une deuxième ligne de quatre bâtiments qui semblent présenter les mêmes caractéristiques. Leur plan est toutefois trop lacunaire.

Isolé à $15 \mathrm{~m}$ à l'est du premier alignement, le bâtiment $\mathrm{D}$ présente un corps principal de $21 \mathrm{~m}$ x 14,70 $\mathrm{m}$ avec neuf piliers disposés en U soutenant un étage et précédé d'un portail encadré par deux pavillons. Ce plan est communément interprété comme une grange (Ferdière, 1988). Il faut enfin signaler un petit édifice à peu près carré au sud-est du précédent, à proximité du tracé supposé du mur d'enclos.

\section{La villa sur la rive gauche du Girondan}

Malgré les lacunes dans la documentation, il semble que la plaine du Girondan opère une coupure nette dans la villa. Quelques constructions y sont disséminées dans un environnement plus ou moins laissé aux divagations du bétail et aux caprices de la rivière. La rivière n'a en tout cas qu'une fonction utilitaire. Il n'en est tiré aucun parti d'ordre esthétique, comme cela pu être le cas dans la villa du Pont-d'Oly à Jurançon dans les Pyrénées-Atlantiques (Balmelle, 2001).

À l'ouest du Girondan la villa est encore mal connue. À l'exception du mur d'enceinte, un seul bâtiment de $6 \mathrm{~m}$ de côté non stratifié a été fouillé (fig. 5, parcelle AO99). L'isolement actuel de cette trouvaille située à $140 \mathrm{~m}$ du Girondan ne doit toutefois pas faire illusion. Des informations orales font état d'autres constructions recoupées lors de travaux et des concentrations de matériel ont été observées dans plusieurs parcelles, notamment en périphérie de l'espace enclos.

\section{LE DOMAINE ET SES PRODUCTIONS}

\section{L'occupation de la campagne autour de la villa}

Si la première villa exploitait les zones humides, il faut attendre le milieu du I ${ }^{\mathrm{er}}$ s. apr. J.-C. pour voir se multiplier des traces indubitables de mise en valeur sur les terrains secs du nord et de l'ouest de la commune (fig. 12). Les fermes fondées à La Tène finale restent toutes en activité à l'exception notable de la villa du Pré-Moly à Leyrieu qui paraissait pourtant auparavant le seul gisement d'une importance comparable à la villa du Vernai. D'autres fermes apparaissent sur des sites vierges et illustrent l'exploitation de nouveaux types de sols. Pour la première fois, la terrasse fersiallitique au bord du Rhône est occupée. Les implantations se multiplient aux abords du marais de la Besseye, au sud, mais pas autour du marais du Grand-Plan. Le site créé sur la butte morainique semble en effet plutôt évoquer un atelier métallurgique.

Parallèlement à ces points de peuplements, se met en place sur tout le territoire communal un semis à peu près uniformément réparti de dépendances matérialisées par de petites concentrations de débris très pauvres en céramiques. Plusieurs ateliers forment une troisième catégorie de gisements essentiellement localisés au bord des voies de communication. Il faut toutefois relativiser leur importance dans l'économie domaniale. L'atelier de tuilier du Terreau est abandonné au plus tard au début du $\mathrm{II}^{\mathrm{e}} \mathrm{s}$. et rien ne permet d'affirmer que l'atelier de potiers de la Plaine à Chavanoz dépendait bien de la villa.

La mainmise de cette dernière sur la campagne environnante se manifeste par le maintien de tous les autres sites habités dans un statut de simple ferme sur un rayon de plusieurs kilomètres. La plus proche villa a été repérée à $3 \mathrm{~km}$ à l'ouest, sur la commune de Tignieu. À l'est, la disparition de la villa du Pré-Moly renvoie à celle supposée exister sous le hameau de Saint-Étienne à Hières-sur-Amby, distante de près de $6 \mathrm{~km}$. Il semble donc que la sphère d'influence de la villa s'étende à l'ouest jusqu'à la Girine et, au sud, au-delà de la cuvette de la Besseye. Les limites à l'est sont plus floues et pourraient se situer quelque part sur la commune de Verna, au-delà du Pré-Moly où se situent d'importantes résurgences karstiques. Le domaine contrôlé par la villa couvrirait ainsi entre 2500 et 3000 ha à l'intérieur desquels les terrains humides représentent moins du quart de la surface totale.

\section{Le marais aux $\mathrm{I}^{\mathrm{er}}$ et $\mathrm{II}^{\mathrm{e}} \mathrm{s}$.}

Les évolutions architecturales et environnementales ne peuvent plus être corrélées pendant les quatre premiers siècles de notre ère (Berger et al., 2003). Ainsi la métamorphose que connaît la villa vers 15 apr. J.-C. ne se traduitelle pas dans le marais. Durant les premières décennies de notre ère, le parcellaire préexistant continue d'être entretenu. Il n'est toutefois pas possible de certifier que son maintien ne s'est accompagné d'aucune modification dans les productions.

La seconde moitié du $\mathrm{I}^{\mathrm{er}} \mathrm{s}$. connaît une période de forte activité hydrologique. Des nappes d'alluvions successives recouvrent le paléosol cultivé depuis la Protohistoire et colmatent tous les fossés drainants (dépôt d'une vingtaine de centimètres en moyenne). Cet épisode dure jusqu'au début $d u \mathrm{II}^{\mathrm{e}} \mathrm{s}$. et pourrait avoir entraîné un abandon des 
cultures. La colonne palynologique réalisée dans la mare (T.2 m.95) montre en tout cas un reboisement grâce à des espèces hygrophiles (aulne, saule, hêtre...) et une nette reprise des Graminées. Cet alluvionnement est peut-être dû à une augmentation des ruissellements consécutive au déboisement et à la mise en culture des versants de la butte morainique, voire même du plateau calcaire où le Girondan prend sa source.

Ce n'est qu'au II $^{\mathrm{e}} \mathrm{s}$. que le marais est repris en main au prix d'une rénovation du réseau de fossés recreusé à partir d'un nouveau paléosol brun-gris, peu gleyfié et plus humifère, développé sur les alluvions récentes. La remise en état du réseau de drainage ne concerne que les terrains situés dans la partie basse du marais, au plus près du site (fig. 13). Les fossés de l'ancien parcellaire nord-sud qui drainaient les rebords de la cuvette ne sont pas recreusés. L'abaissement du niveau de la nappe phréatique par rapport aux périodes antérieures, attesté par une structure granulaire et une porosité biologique plus marquée des sédiments, explique peut-être en partie ce qui ressemble, à première vue, à un abandon partiel du marais. Le drainage naturel du marais, en relation avec l'encaissement du lit du Girondan au cours du $\mathrm{II}^{\mathrm{e}} \mathrm{s}$. aurait réduit la nécessité d'un réseau d'assèchement (pl. VIII hors texte). Pour le moment, un seul fossé de cette phase a fait l'objet d'études palynologiques (T.3 m.10). Comme pour la phase précédente, la proportion de taxons d'arbres, notamment hydrophiles, est importante et le chêne est bien représenté. Par l'association entre les Résédacées qui se développent en milieu non cultivé, la présence de plantain et la prédominance de Cypéracées il est possible de restituer une reconversion de ces espaces en prairie humide. Deux datations radiocarbone homogènes $(1805 \pm 35 \mathrm{BP}$ pour le fossé T.2 m.300 et $1840 \pm 120 \mathrm{BP}$ pour T.3 m.10) obtenues dans différents remplissages de cette phase la situent entre le début du $\mathrm{II}^{\mathrm{e}} \mathrm{s}$. et le début du $\mathrm{IV}^{\mathrm{e}} \mathrm{s}$.

\section{Les productions}

L'économie de la villa connaît des transformations au cours du Haut-Empire. Ces évolutions ne semblent pas corrélées à la rupture sociale que traduit la mutation architecturale de l'époque tibérienne.

Tout au long de cette période, l'élevage semble prendre un essor progressif suggéré par l'accroissement des prairies dans le marais du Grand-Plan ainsi que par la multiplication des fermes exploitant ce milieu (fig. 2 et 20). Les proportions respectives des trois espèces domestiques principales ne se modifient guère avec le changement de villa (fig. 21). Il faut attendre le $\mathrm{II}^{\mathrm{e}} \mathrm{s}$. pour voir s'amorcer une tendance - d'ailleurs plus lisible en poids de viande qu'en nombre de restes - à l'accroissement de la proportion des bovins au détriment des porcins. Cette tendance sur le long terme est toutefois momentanément contrariée à la fin de cette période (tabl. I). Le gabarit des animaux de la triade principale, notamment des bovins, croît pendant ces deux siècles, même si le profil fourni par les restes consommés peut différer de celui du cheptel réellement élevé (fig. 21). Les modifications les plus sensibles concernent toutefois les espèces secondaires. Le changement de nature de la chasse transparaît avec la disparition du petit gibier d'eau et du lièvre. En revanche apparaissent le chevreuil, le sanglier et, surtout, le cerf qui, rapidement, occupe en nombre de restes une place notable. Cette irruption d'une population sélectionnée conduit à évoquer l'hypothèse d'un élevage en semi-liberté.

À l'inverse, la place consacrée à la céréaliculture se réduit dans le marais. Ce phénomène doit toutefois être relativisé par l'extension conséquente des cultures sur les sols secs et par l'agrandissement pressenti du domaine.

Le milieu du $\mathrm{I}^{\mathrm{er}} \mathrm{s}$. voit surtout une diversification des productions agricoles en relation avec l'exploitation de types de sols jusqu’alors négligés.

L'arboriculture se développe. Le noyer (Juglans regia) apparaît à cette époque, en quantités plus ou moins significatives dans les diagrammes polliniques (T.45 phase 9, fig. 10). Des prélèvements carpologiques ont été effectués dans des zones de rejets en périphérie du site. Le plus riche en témoins de productions agricoles est sans conteste l'assemblage des $\mathrm{I}^{\mathrm{er}}-\mathrm{II}^{\mathrm{e}}$ s. apr. J.-C. provenant de T.57, une tranchée localisée immédiatement au nord de la villa à proximité du canal de drainage du marais. Les pépins de raisin (Vitis vinifera) occupent une position nettement dominante. La vigne est associée à d'autres arbres cultivés : le noyer (Juglans regia), les pruniers (Prunus type domestica et Prunus type insititia), éventuellement le noisetier (Corylus avellana) et le cerisier doux ou merisier (Prunus type avium), ces deux derniers pouvant également être sauvages. Aux côtés de ces arbres fruitiers se rencontre un petit cortège d'arbustes des haies et des lisières, composé du cornouiller sanguin (Cornus sanguinea), du prunellier (Prunus type spinosa), du sureau noir (Sambucus nigra) et de la viorne lantane (Viburnum lantana). La gourde calebasse (Lagenaria siceraria) est la seule plante annuelle cultivée.

On peut supposer que cet assemblage est constitué de semences naturellement drainées à l'intérieur du canal et 


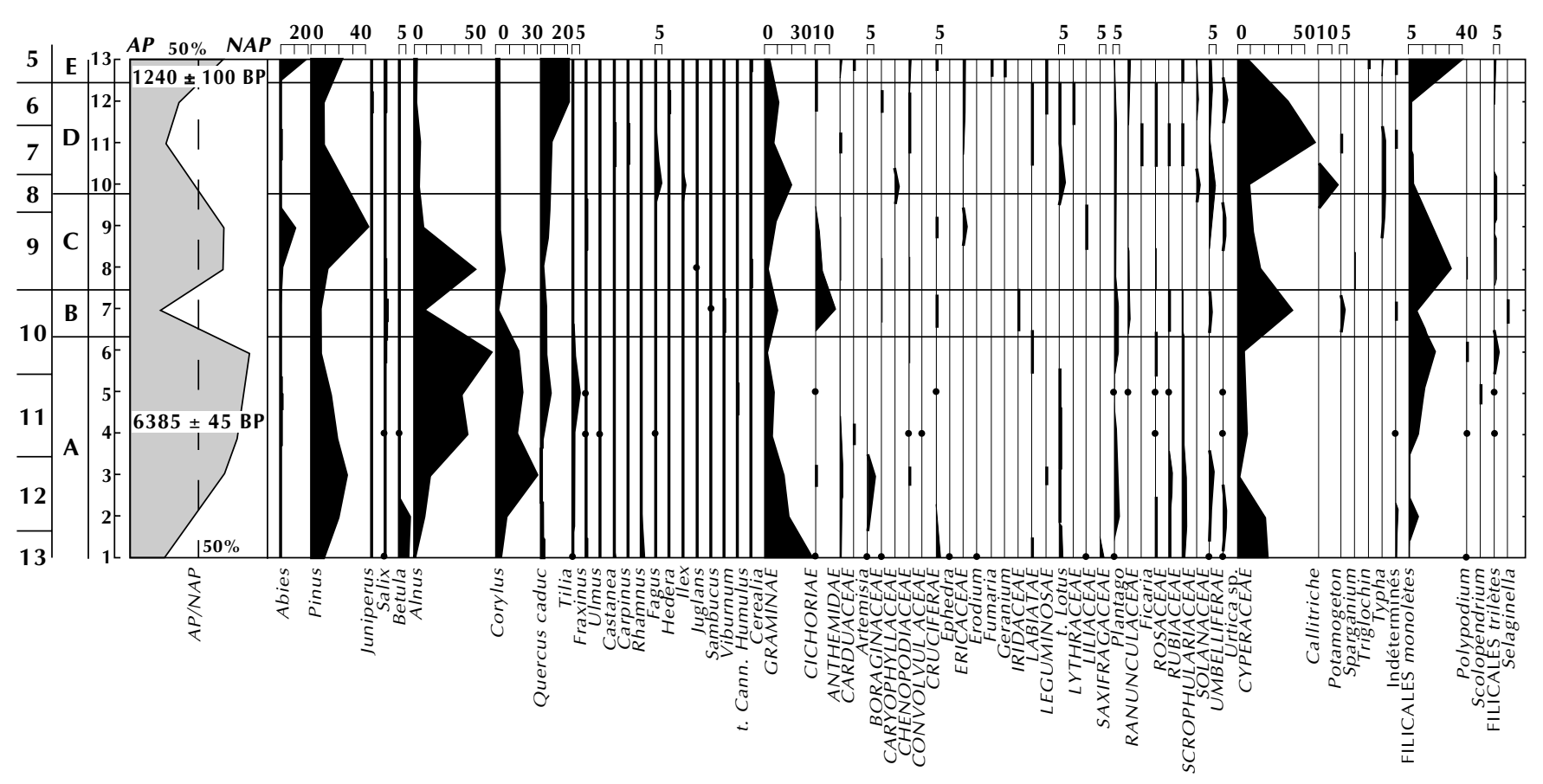

Fig. 20 - Diagramme pollinique du marais du Grand-Plan (T.2 m.94,5) (DAO M. Bui Thi, CNRS).

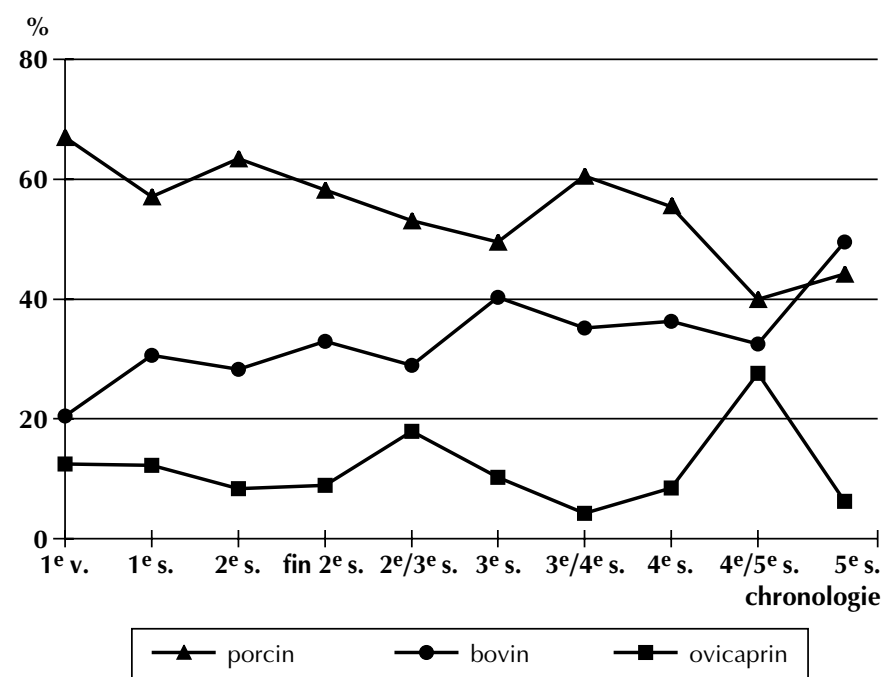

Fig. 21 - Faune: évolution chronologique des restes de taxons de la triade domestique: $1^{e} v$., villa précoce; s., siècle (DAO V. Forest).

qu'il est représentatif de l'environnement stationnel. Une vigne aurait alors pu pousser à proximité, vraisemblablement sur la butte morainique, en association avec d'autres arbres fruitiers (noyer, prunier), éventuellement séparée du cours d'eau par une haie. Il est aussi possible que l'assemblage, essentiellement constitué de fruits comestibles, possède une origine détritique. L'absence de céramique associée à la surreprésentation de taxons de raisin impliquerait que ces rejets soient pour partie liés à des installations viticoles. Cette hypothèse détritique est confortée par la présence de la gourde calebasse, une plante d'origine tropicale dont la culture requiert de la chaleur et ne tolère pas le gel. Elle a donc vraisemblablement été importée au Vernai et représente donc plutôt un déchet rejeté, même si elle a pu être occasionnellement cultivée, en des lieux ensoleillés particulièrement propices, jusque dans le nord de la France et en Allemagne.

Les données sur l'artisanat, au moins dans la villa, sont peu nombreuses. Une activité textile pourrait être attestée par la découverte de nombreux pesons de tisserands ainsi que de pots provenant du secteur 3 et contenant des colorants qui n'ont malheureusement pas été analysés. La culture du chanvre paraît certaine dans la vallée du Girondan (fig. 9, échantillons 14 à 18). À l'extérieur du site, l'importance et la durée de vie des ateliers de tuiliers situés au nord de la commune et de la forge retrouvée sur la butte morainique ne sont pas quantifiables. La part de ces nouvelles cultures, notamment la vigne, et de l'artisanat dans l'économie de la villa au détriment du couple céréaleélevage bovin reste elle-même à évaluer. 
La redistribution des sites autour de la villa pourrait attester une transformation du système productif du domaine au profit d'un faire-valoir indirect, ce que semble confirmer l'assez faible quantité de bâtiments détectés dans le secteur productif.

\section{UNE VILLA EN MUTATION (III $\left.{ }^{\mathrm{e}}-\mathrm{V}^{\mathrm{e}} \mathrm{S}.\right)$}

Au début du $\mathrm{III}^{\mathrm{e}} \mathrm{s}$. une crise hydrologique majeure se fait sentir dans les zones humides du domaine et contraint les occupants à une réorganisation de la partie résidentielle.

\section{L'HABITAT}

L'assiette de la pars urbana est déplacée sur la partie la plus haute de la levée alluviale au-dessus de la cote 202,50 m NGF. Ce glissement vers le sud n'est pas le symptôme d'une décadence de la villa ni d'une rétractation des surfaces bâties.

\section{La réfection de la cour}

La péjoration hydrologique constatée au III $^{\mathrm{e}} \mathrm{s}$. entraîne la restructuration de l'extrémité nord de la villa, plus basse d'environ $1,50 \mathrm{~m}$ que le reste de la partie résidentielle. La cour nord est alors réduite et le bâtiment XIIA, qui la bordait à l'ouest, rasé. Afin de lutter contre le regain d'humidité, elle est non seulement rehaussée de plus de $50 \mathrm{~cm}$ par des remblais mais aussi drainée. Ce nouvel espace, où quelques petits bâtiments monocellulaires sont construits entre le $\mathrm{III}^{\mathrm{e}}$ et le $\mathrm{V}^{\mathrm{e}} \mathrm{s}$., devient une arrière-cour des thermes qui sont mis en place immédiatement au sud.

\section{Le nord de la partie résidentielle au Bas-Empire}

C'est dans ce secteur que se concrétise le plus clairement le dynamisme qui se manifeste sur le site au cours des derniers siècles de l'Empire (fig. 17, phases 4 et 5). Une quatrième phase de travaux va en modifier profondément l'aspect et peut-être la fonction. Dans la partie orientale, après arasement de l'édifice antérieur, un bâtiment thermal à itinéraire rétrograde est édifié. Le circuit comporte un frigidarium de $22 \mathrm{~m}^{2}$ (PIVC), flanqué à l'ouest d'une natatio à la cuve plaquée de marbre de 2,10 m x 3,90 m et prolongé au nord par un tepidarium et par une cella soliaris de même largeur $(1,85 \mathrm{~m})$ en opus testaceum. Cette dernière pièce comporte un solium de $5 \mathrm{~m}^{2}$ à l'ouest. Le praefurnium initial (V1345) était installé au nord de PIVA, dans l'axe de symétrie des pièces. À côté, on trouve les restes très dégradés d'un massif bordé d'une évacuation, peut-être le support d'une chaudière (fig. 17).

La palestre IIIC est progressivement embellie; une natatio de $6 \mathrm{~m}$ × $20 \mathrm{~m}$ (V902), profonde de $1,90 \mathrm{~m}, \mathrm{y}$ est creusée à la fin du II $^{\mathrm{e}} \mathrm{s}$. ou plutôt au début du $\mathrm{III}^{\mathrm{e}} \mathrm{s}$. Ses parois étaient habillées de dalles de calcaire blanc et son fond, parcouru par des drains périphériques en imbrices, était revêtu d'un opus tesselatum blanc. L'eau provenait d'une borne-fontaine située à $1 \mathrm{~m}$ de l'angle sud-est du bassin et s'évacuait dans le collecteur V911, qui subit à ce moment des travaux de réfection.

Dans un cinquième temps, la palestre est rétrécie par la construction de galeries à portiques (IVE et IVF), larges respectivement de 2,50 m et 2,95 m. Le libage V1335 est une fondation posée sur un hérisson large de $0,65 \mathrm{~m}$ et profonde de $0,60 \mathrm{~m}$ à appareil assisé pseudo-régulier similaire à l'abside de la pièce A12 au pied de l'église.

Le dernier remaniement dans ce secteur est la construction, au plus tôt au $\mathrm{V}^{\mathrm{e}} \mathrm{s}$., à l'emplacement du praefurnium V1345, d'un bassin (V1306) de 2,50 $\mathrm{m}$ de diamètre (fig. 17, phase 4). Un nouveau foyer, construit avec un bloc d'entablement de réemploi, est installé au milieu du côté oriental de la cella solaris (V1307).

\section{L'extrémité méridionale de la pars urbana: salle de réception à abside ou lieu de culte chrétien?}

La campagne de construction du portique autour de la piscine est aussi l'occasion de transformations apportées à l'extrémité méridionale de la partie résidentielle (parcelle AI181). Dans sa partie fouillée, ce secteur n'avait été affecté depuis le $\mathrm{II}^{\mathrm{e}} \mathrm{s}$. que par l'installation d'un caniveau en blocs de calcaire bajocien le long du portique et par un déplacement du praefurnium de l'hypocauste de la pièce A12, transféré à la fin du $\mathrm{II}^{\mathrm{e}} \mathrm{s}$. ou au $\mathrm{III}^{\mathrm{e}} \mathrm{s}$. hors du bâtiment, face à l'axe de la pièce à la place de la fontaine V184 (fig. 15, phase 4).

Ce deuxième praefurnium est condamné par l'adjonction dans le prolongement de A12 d'une abside A13 en arc de cercle outrepassé de $7 \mathrm{~m}$ hors tout (espace intérieur: 5,60 m), associée à un contrefort carré (fig. 15, phase 5), dont le sol, une chape de mortier de tuileau, prolonge exactement celui de la pièce A12. Le matériel retrouvé dans la tranchée de fondation de l'abside ou dans les remblais préparatoires ne permet qu'une datation assez floue à l'intérieur du $\mathrm{IV}^{\mathrm{e}} \mathrm{s}$. Cette association d'une abside en arc outrepassé à une pièce résidentielle peut, par ses dimensions 
et sa chronologie, être comparée à celle de la villa de la Ramière à Roquemaure dans le Gard (Barberan et al., 2002) et semble s'inscrire dans la longue série des pièces de réception à abside centrale répandue au Bas-Empire, notamment dans le Sud-Ouest (Balmelle, 2001).

Pourtant, divers indices conduisent à s'interroger sur cette interprétation et à proposer de voir dans cet ensemble l'illustration de la christianisation des propriétaires.

Le premier argument allant dans ce sens est l'évolution ultérieure de ce secteur qui voit la mise en place précoce de l'église actuelle. Les points de comparaison que fournissent certains monuments paléochrétiens comme, par exemple, la basilique funéraire de Viviers en Ardèche (Esquieu dir., 1988) ou, plus proche, l'état primitif de l'église de Larina à Hières-sur-Amby (Porte, 2002) contribuent aussi à étoffer cette hypothèse. Un dernier argument est l'installation au milieu du VI ${ }^{\mathrm{e}} \mathrm{s}$. (datation à 1 sigma), voire dès la seconde moitié du V $\mathrm{V}^{\mathrm{e}} \mathrm{s}$. (datation à 2 sigmas: $1510 \pm 30 \mathrm{BP}$ ), d'une tombe d'un défunt au niveau social élevé, dans l'angle nord de l'abside. Un coffre rectangulaire complet composé de grosses pierres récupérées sur la villa est installé dans un caveau soigneusement détouré. Le couvercle monolithe est ajusté exactement au niveau du sol de l'abside. Cette tombe est la seule de ce type retrouvée sur le site et tout laisse à penser qu'elle a été installée sciemment dans l'abside.

\section{LE SECTEUR PRODUCTIF}

\section{Le secteur XX}

La multiplication des crues du Girondan à partir du $\mathrm{III}^{\mathrm{e}} \mathrm{s}$. va nécessiter la réfection du bâtiment fouillé sur le bord de la rivière (fig. 19). On constate le dépôt de remblais riches en céramique associé à des niveaux de travail. Un incendie a détruit cette construction au cours de la seconde moitié du $\mathrm{III}^{\mathrm{e}}$ s. À l'exception d'un sac de vesces, le feu n'a figé sur place aucun témoin de sa fonction.

Du matériel, notamment une demi-douzaine de monnaies, attestant une occupation tardive avait aussi été relevé dans les deux bâtiments du secteur 3, fouillés en 1968.

\section{La plaine du Girondan jusqu'à la fin de l'Antiquité (III ${ }^{\mathrm{e}}-\mathrm{VI}^{\mathrm{e}}$ s.)}

À la fin du $\mathrm{II}^{\mathrm{e}}$ s. ou au début du $\mathrm{III}^{\mathrm{e}}$ s., une nouvelle phase d'accumulation de sédiments gravelo-sableux rehausse le plancher de la rivière de près de $50 \mathrm{~cm}$. Une série de crues répand des niveaux d'alluvions fines dans la plaine d'inondation (pl. VIII hors texte).

Cet épisode s'interromptà la fin du III ${ }^{\mathrm{e}}$ s. sous l'effet d'une modification du régime de la rivière dont le lit va à nouveau s'encaisser, puis du fait d'une tentative de stabilisation et de contrôle, la plus importante que le Girondan ait connu depuis la fin du $\mathrm{I}^{\mathrm{er}} \mathrm{s}$. av. J.-C. La rive est consolidée par un remblai composé de pierres, blocs et tegulae maintenu par un alignement de petits pieux et de palplanches (pl. VIII hors texte). Cet aménagement implanté dans le lit à plus de $3 \mathrm{~m}$ de l'ancienne berge repousse le cours de la rivière vers l'ouest, à son emplacement actuel. Il est doublé par un mur maçonné qui pourrait servir à border un chemin longeant la rive. La reprise en main du secteur se concrétise surtout par la construction d'un gros drain collecteur destiné à assécher l'ancien bras mort du Girondan qui se matérialise encore par une légère dépression. Un sol brunifié associé à un important réseau de racines se développe dans ce bras.

Le diagramme pollinique de la tranchée 56 fournit, pour la fin de l'Antiquité (fig. 9, échantillons 7 à 15), des éléments sur l'aspect des zones humides en périphérie de la villa. La faible proportion des taxons d'arbres est l'indice d'un paysage largement ouvert où la multiplication des aulnes, des noisetiers, et la présence de saules et de peupliers signale une reconstitution au moins locale de la ripisylve. Les plantes adventices associées aux habitats abondent. La présence, déjà signalée en moindre proportion pour le Haut-Empire, de l'oseille et du plantain associés à des Poacées et à des Cypéracées marque la proximité de prairies humides et signe le regain d'importance que connaît l'élevage. La réapparition des pollens de céréales associés à des vesces marque la mise en culture de champs proches, peut-être au bord de la rivière, plutôt que l'extension des surfaces emblavées dans le domaine.

L'importance du taux d'herbacées ne permet pas de conclure à la désertion et à la revégétalisation des abords de la rivière dans l'enceinte de la villa. Au cours du $\mathrm{III}^{\mathrm{e}} \mathrm{s}$., on constate une rénovation du bâtiment XXA qui jouxte la tranchée, ainsi que des empierrements de sols aux alentours de celui-ci. Les fouilles du secteur 3 situé $70 \mathrm{~m}$ plus au sudest avaient, elles aussi, livré du matériel des $\mathrm{III}^{\mathrm{e}}$ et $\mathrm{IV}^{\mathrm{e}} \mathrm{s}$.

Cette phase d'accalmie hydraulique prend fin avec un nouvel épisode d'accumulation de sédiments grossiers, accompagné de crues violentes qui vont submerger l'aménagement de la berge. Une date radiocarbone permet de dater ce nouveau dérèglement hydrologique immédiatement après l'abandon du fossé de drainage (1525 \pm 30 BP), étudié 
dans le sondage 25. Cette crise non contenue accompagne la disparition de la villa.

\section{L'UTILISATION DU MARAIS DU III ${ }^{\mathrm{e}}$ AU $\mathbf{V}^{\mathrm{e}} \mathrm{S}$.}

Faute d'une restauration intégrale du réseau de drainage, on ne dispose d'une information sédimentaire que sur une partie du marais. Pour tout le nord et le nord-est de celuici, la seule source de documentation est la mare recoupée en T.2 m.94,5 dans laquelle le calage de l'accumulation sédimentaire est imprécis. Les trois niveaux intercalés entre le milieu de l'Antiquité et la fin du haut Moyen Âge présentent des similitudes morphologiques. Cette dépression est toujours en eau ainsi que le montrent le dépôt d'un sédiment crayeux et le développement de Callitriche, une plante totalement immergée, requérant un plan d'eau libre et claire. Les terres aux alentours continuent d'être entretenues, l'essartage qui déclenche une érosion importante étant attesté par la présence significative de charbons et de Chaetomium, un champignon lignicole et carbonicole. Les pics de cyanobactéries, comme Anabanea et Aphanizomenon, révélatrices d'apports de composés nitrogènes riches en phosphore ayant pollué la nappe phréatique, illustrent l'accroissement de l'élevage. À côté du bœuf et du porc, le cerf connaît une progression constante dans les lots de la fin de l'Antiquité (entre $9 \%$ et $13 \%$ du poids de viande). On peut se demander si l'homogénéité de la population consommée (essentiellement des adultes mâles) n'atteste pas une chasse sur une population élevée en semi-liberté dans les prairies humides du marais.

Dans la partie encore drainée, on constate d'abord la reprise des accumulations dans les fossés curés au début du $\mathrm{II}^{\mathrm{e}}$ s. puis une nouvelle phase d'alluvionnements (fig. 13). Celle-ci peut être raccordée à la phase de débordements du $\mathrm{III}^{\mathrm{e}} \mathrm{s}$., déjà identifiée sur le site grâce à plusieurs datations radiocarbone effectuées dans les colmatages $(1805 \pm 35 \mathrm{BP}$ et $1840 \pm 120 \mathrm{BP})$. Une quatrième et ultime tentative d'entretien des fossés remet en état une partie du réseau. Les sédiments de remplissage limono-argileux très fins subissent un très fort enrichissement en matière organique confirmé par la présence des cyanobactéries Aphanizomenon et Anabanea, associées à des milieux eutrophiques. La structure prismatique de ces sédiments révèle un fort battement saisonnier de la nappe phréatique. Tous ces traits suggèrent donc des herbages humides consacrés à l'élevage. Le diagramme pollinique signale néanmoins la réapparition des céréales associées au plantain et à des adventices à la fin de cet épisode. Ces échantillons ayant été prélevés au cœur du marais, à une distance respectable des versants, il faut donc envisager la possibilité d'une remise en culture de certains terrains proches de la villa à la fin de l'Antiquité.

Les datations des remplissages immédiatement postérieurs à la dernière tentative de curage sont homogènes. Les dates radiocarbone obtenues $(1565 \pm 50 \mathrm{BP}$ et $1525 \pm 50 \mathrm{BP})$ apparaissent synchrones de l'abandon des fossés bordant le Girondan. L'abandon de tout essai de contrôle de l'humidité dans le marais peut être daté de la fin de la période antique ou du début de la période suivante $\left(\mathrm{V}^{\mathrm{e}}-\mathrm{VI}^{\mathrm{e}} \mathrm{s}.\right)$. Une succession d'alluvionnements limoneux scelle définitivement tous les canaux et recouvre alors le sol de culture, postérieurement au $\mathrm{VI}^{\mathrm{e}} \mathrm{s}$.

\section{L'ÉCONOMIE DE LA VILLA AU BAS-EMPIRE}

Les modifications et regroupements que peut connaître le domaine au Bas-Empire sont difficiles à évaluer. Tous les sites interprétés comme des fermes pour les deux premiers siècles de notre ère livrent du matériel postérieur mais souvent en quantité bien moindre. Au-delà de la sphère d'influence de la villa on note le maintien ou la réimplantation de sites sans doute importants (fig. 22). L'ancien établissement du Pré-Moly est ainsi réoccupé au IV ${ }^{\mathrm{e}} \mathrm{s}$. On ne constate donc pas une désertification régionale qui aurait été propice à l'extension du domaine.

La vocation agricole s'affirme au détriment des activités artisanales dont ne subsistent que peu de traces. Seul perdure l'atelier de métallurgie situé sur la butte, à quelques centaines de mètres du site ainsi que, sur les bords du Girondan, le témoignage du rouissage du chanvre. Malheureusement les niveaux du Bas-Empire des bâtiments économiques n'ont guère livré de matériel représentatif d'une activité textile.

L'importance de l'élevage est peut-être surévaluée, d'une part à cause du hasard des découvertes qui ont fourni des dépotoirs de la fin de l'Antiquité et, d'autre part, du fait de la spécialisation progressive du marais qui ne représente pourtant qu'une fraction du domaine. Il n'est donc pas possible de comparer les effectifs de restes consommés au Bas-Empire avec ceux du cheptel du début de notre ère.

On peut distinguer deux tournants dans la gestion de la faune au Bas-Empire (tabl. I). Le premier se place au III ${ }^{\mathrm{e}} \mathrm{s}$. et voit l'arrêt, du moins en nombre d'individus, du processus de diminution des porcins dans l'élevage. La part de la viande bovine reste toutefois en augmentation constante 


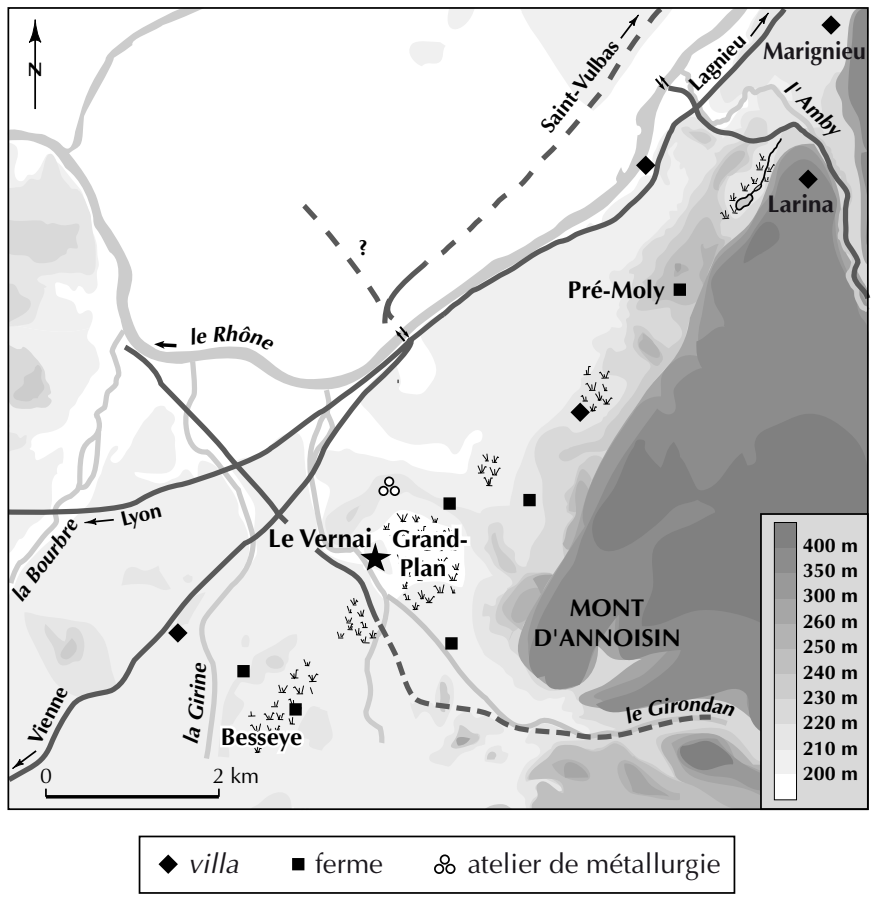

Fig. 22 - Carte des abords du site du Vernai aux III -V $V^{e}$. (DAO R. Royet, SRA Rhône-Alpes).

et connaîtra son importance maximale durant le $\mathrm{IV}^{\mathrm{e}} \mathrm{s}$. Alors que, depuis La Tène finale, la proportion entre bovins et porcins était stabilisée à $60 \%$ contre $40 \%$ pour les porcins, les bovins représentent près de $70 \%$ au IV ${ }^{\mathrm{e}} \mathrm{s}$. Le début du Bas-Empire connaît aussi une augmentation de la proportion du nombre de restes de cerf. Le second tournant correspond à l'ultime phase de fonctionnement de la villa. Au cours du $\mathrm{V}^{\mathrm{e}} \mathrm{s}$., la corpulence des bovins diminue même si leur effectif augmente. Ce phénomène est précoce par rapport à d'autres sites de la région où il n'est constaté qu'au cours des VII ${ }^{\mathrm{e}}$-VIII ${ }^{\mathrm{e}}$ s. (Forest et al., 2001). La fin de l'Antiquité voit diminuer fortement l'importance du cheptel porcin dont l'apport carné ne dépasse plus guère $20 \%$ du total fourni par les espèces domestiques.

La céréaliculture et l'élevage restent, dans l'économie du domaine du Bas-Empire, concurrencés ou complétés par des productions agricoles à forte valeur marchande comme la vigne, le noyer ou le châtaignier dont la présence s'accentue dans les diagrammes palynologiques.

Rien n'atteste donc jusqu'au $V^{\mathrm{e}} \mathrm{s}$. un repli de l'exploitation sur elle-même et une tentation autarcique qui pourraient être la marque d'une phase de déclin.

\section{UNE EXPLOITATION DU HAUT MOYEN ÂGE}

Il existe actuellement un hiatus entre la disparition de la villa à la fin $\mathrm{du} \mathrm{V}^{\mathrm{e}}$ s. et la construction de l'exploitation qui lui succède, à la fin du VI ${ }^{\mathrm{e}}$ s. ou au début du VII ${ }^{\mathrm{e}}$ s. (fig. 23). La rupture entre ces deux établissements semble profonde, même si certains vestiges de la villa encore en élévation sont réemployés dans la nouvelle construction. Les installations hydrauliques, égouts ou drains ne sont pas remis en fonctionnement. Les habitants ne vont plus occuper que la partie sommitale de la butte qui est alors organisée en deux pôles distincts: au centre du gisement, une église et son cimetière prennent la suite de la chapelle de la villa; près de $100 \mathrm{~m}$ à l'ouest, un grand bâtiment d'habitat associé à quelques dépendances, est édifié sur les ruines de la villa tardive (fig. 13 et 23).

\section{L'HABITAT}

Un bâtiment de près de $350 \mathrm{~m}^{2}$ est installé sur les ruines de la villa dont il réemploie non seulement les matériaux mais aussi certains murs encore en élévation. La majeure partie de cette construction est toutefois bâtie en recourant à un appareil en épi dont ne subsistent que deux ou trois assises, larges de $0,75 \mathrm{~m}$, de pierres et tuiles maçonnées au mortier de chaux posées sur une semelle liée à la terre. Les sols, les élévations et la couverture, vraisemblablement en matériaux légers, n’ont laissé aucune trace. Cet édifice est organisé en trois bandes principales.

Son corps principal de $167 \mathrm{~m}^{2}$ (21,55 m x 7,75 m), divisé en cinq pièces, présentait une organisation linéaire. Seule sa partie médiane, large de $3,75 \mathrm{~m}$, se compose de deux pièces échelonnées en profondeur. L'entrée et la distribution de la circulation s'effectue par un vestibule de $7 \mathrm{~m}^{2}$ (P1), au sol en mortier de tuileau très maigre, le seul conservé dans cette construction. Elle donne au nord sur une pièce (P2), à peu près carrée, de $14,5 \mathrm{~m}^{2}$, qui présente une stratigraphie des remblais relativement complexe mais sans rapport avec son usage. Une dépression créée par le tassement différentiel de la fosse V1279, sous-jacente, a été colmatée avec une couche de tuiles brisées, puis avec un nouveau remblai de pierres et surtout de tuiles. La fonction de son aménagement interne, une série de quatre poteaux fichés contre les murs, n’a pu être élucidée.

Deux pièces en enfilade (P3 et $\mathrm{P} 4$ ), de $28 \mathrm{~m}^{2}$, se partagent équitablement la partie orientale du bâtiment. De leur stratigraphie, seuls subsistent des fragments de 


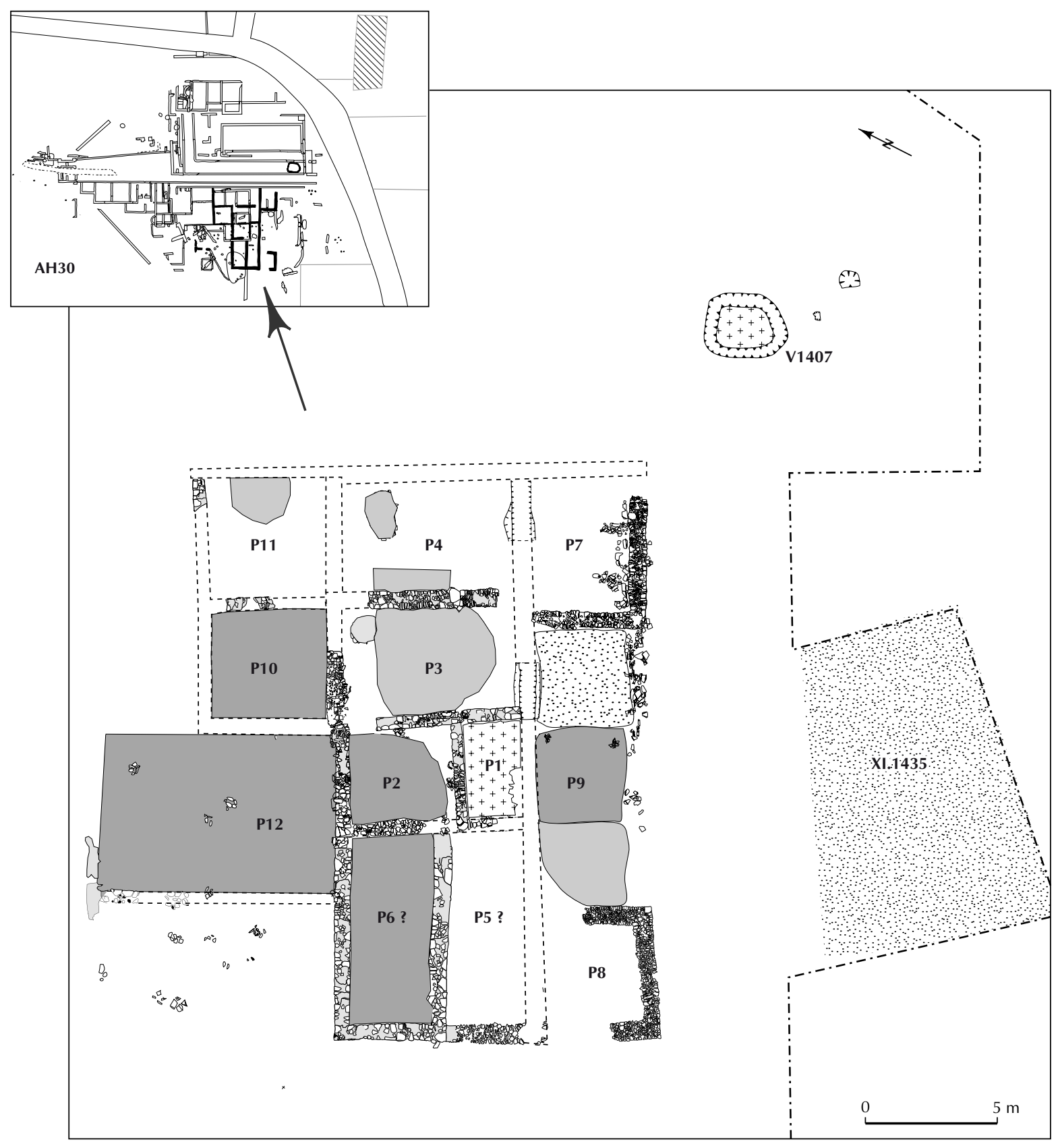

\begin{tabular}{|lll|}
\hline$-\cdot-$ limite de fouille & $\square$ radier de pierre & $\square$ sol en gravier \\
\hdashline.--- tranchée de récupération & $\square$ radier de tuile & mortier de tuileau \\
\hline
\end{tabular}

Fig. 23 - Habitat du haut Moyen Âge dans la parcelle AH30, secteur XI du site du Vernai (DAO R. Royet, SRA Rhône-Alpes). 

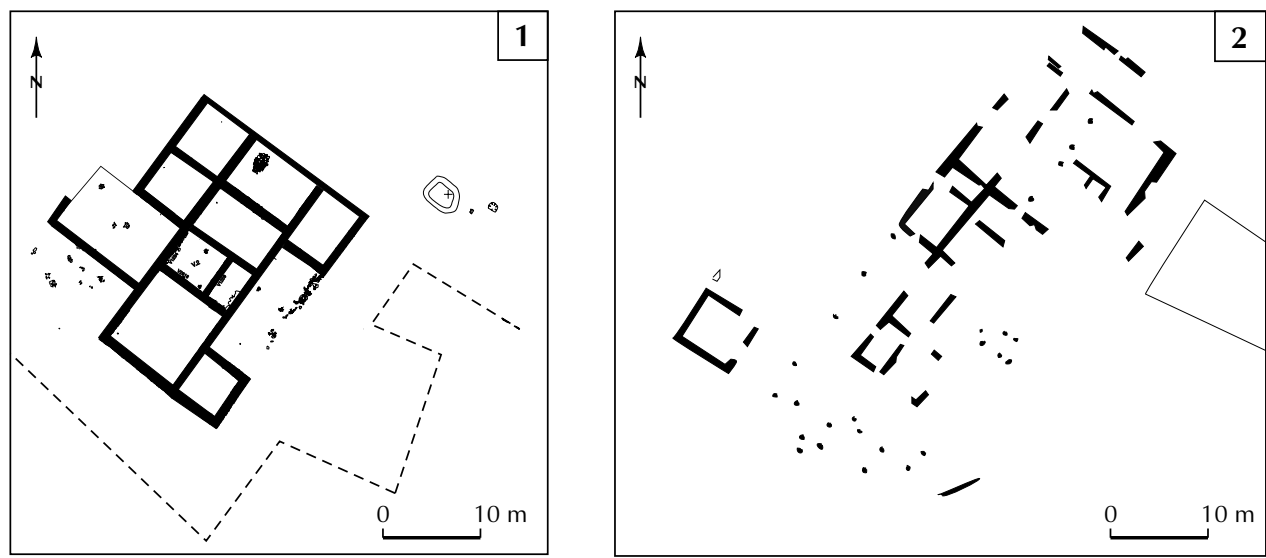

Fig. 24 - Comparaison de différents habitats du haut Moyen Âge: 1, le Vernai à Saint-Romain-deJalionas (Isère) ; 2, le Bivan à L'Albenc (Isère) ; 3, bâtiment 1, Larina à Hières-sur-Amby (Isère) ; 4, 54 rue Pierre-Audry à Lyon (Rhône) (DAO R. Royet, SRA Rhône-Alpes).
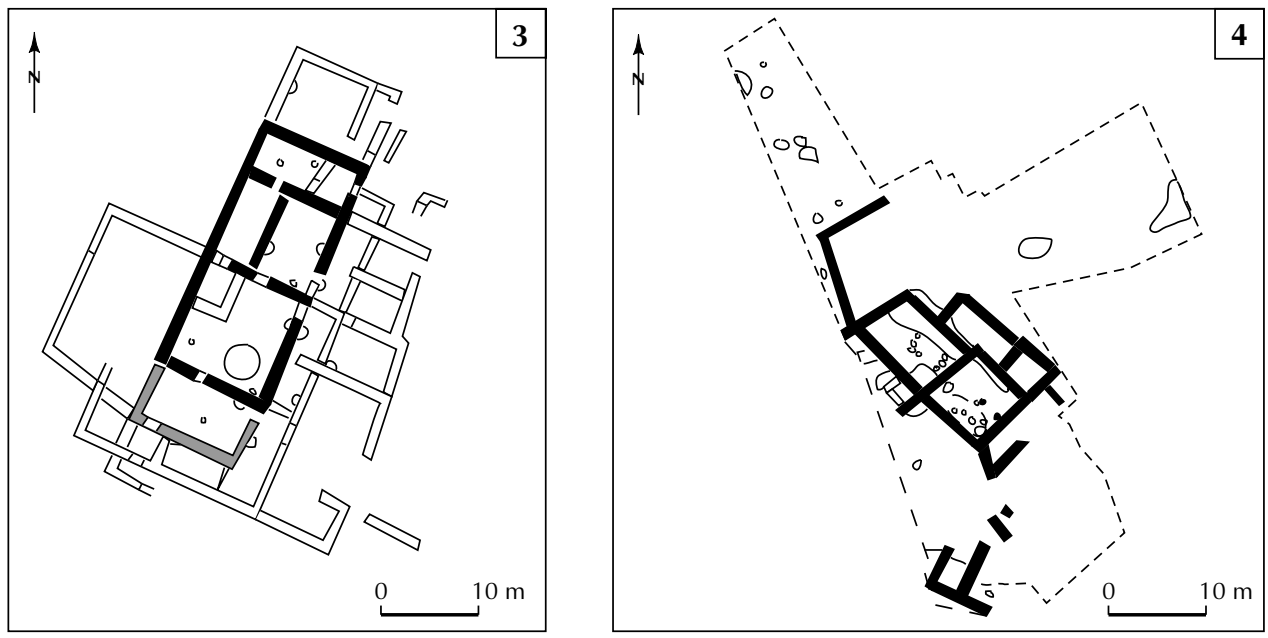

radiers de pierres arasés à la cote 202,60 m NGF. Enfin, dans l'angle nord-est de la pièce P3 est conservé un massif de mortier circulaire de $1 \mathrm{~m}$ de diamètre qui était peut-être la base d'un foyer.

À l'ouest de $\mathrm{P} 1$, la grande pièce $\mathrm{P} 5 / 6$, de $58 \mathrm{~m}^{2}$, peut être interprétée comme une aula comparable à des exemples fournis par les habitats de Larina et du 54 rue Pierre-Audry à Lyon (Faure-Boucharlat dir., 2001) (fig. 24). Ici, comme en P2, l'apport d'un remblai localisé s'explique par un tassement du dépotoir sous-jacent V1279.

$\mathrm{Au}$ nord de la bande principale, les pièces $\mathrm{P} 10$ et $\mathrm{P} 11$ offrent la particularité d'être des espaces de la villa galloromaine dont les sols sont exhaussés par des radiers de tuiles, pour P10, ou de pierres, pour P11. Rien ne nous renseigne sur leur fonction. Enfin, au nord-ouest, un radier de tuiles perforé de plusieurs trous de poteaux dessine un espace rectangulaire (P12) de $53 \mathrm{~m}^{2}$. Ici encore les sites de Larina, du 54 rue Pierre-Audry à Lyon ou du Bivan à
L'Albenc fournissent un exemple similaire permettant la restitution d'un espace de service partiellement ouvert (Faure-Boucharlat dir., op. cit.).

$\mathrm{Au}$ sud, la façade a été embellie par l'ajout de deux pavillons d'angles (P7 et P8) encadrant une galerie (P9). La division de $\mathrm{P} 9$ en trois parties, sensible par une différence de préparation de sol, conduit à ne pas accorder trop de monumentalité à ce terme qui pourrait induire un parallélisme avec le modèle de la villa à galerie de façade antique.

Parmi la courte série des grands bâtiments résidentiels du haut Moyen Âge du nord de Rhône-Alpes c'est avec celui de Larina qu'il est possible d'établir les comparaisons les plus étroites. Dans les deux cas, on retrouve une importante fragmentation et une même distribution linéaire de l'espace intérieur, la principale différence consistant dans la mise en scène de la façade sud de celui de Saint-Romainde-Jalionas. Le modèle de la villa antique où les fonctions résidentielles et économiques sont dissociées peut sembler 
ici encore prédominant. De la tradition antique manquent toutefois les éléments de chauffage, d'hygiène et de décor.

\section{LES ABORDS DE L'HABITAT}

Les dépendances de cet établissement n'ont pas encore été retrouvées. Les fondations (V1407) d'une hutte de $3,50 \mathrm{~m}^{2}$ signalées à $5 \mathrm{~m}$ à l'est du bâtiment fouillé furent à peu près les seuls vestiges identifiés lors des fouilles anciennes (fig. 23).

Dans des sondages immédiatement à l'ouest du bâtiment principal ainsi que vers P12, plusieurs trous de poteaux ont été relevés sans qu'aucun plan puisse être proposé. Enfin il convient de signaler, au sud du bâtiment, un sol de graviers et petits galets calibrés mélangés à de petits morceaux de tegulae (XI.1435), installé dans une dépression qui était peut-être un chemin creux se dirigeant vers l'église.

\section{LE LIT MAJEUR DU GIRONDAN}

À cette époque, la plaine du Girondan ne connaît plus qu'une fréquentation épisodique. La crise hydrologique (pl. VIII hors texte, phase 10c), évoquée lors de la phase antérieure et caractérisée par des dépôts alluviaux hétérogènes grossiers, doit prendre fin entre le $\mathrm{VI}^{\mathrm{e}} \mathrm{s}$. (derniers colmatages de fossés vers $1525 \mathrm{BP}$ ) et le VIII ${ }^{\mathrm{e}} \mathrm{s}$. (1240 BP pour la phase calme à tourbe).

Ultérieurement, un paléosol humique brun à noir se constitue progressivement, non seulement dans le lit de la rivière mais aussi sur le site et dans le marais, où il se trouve associé à des niveaux tourbeux dans les parties déprimées (pl. VIII hors texte, phase 11). Ce paléosol et cette tourbe dans le lit majeur du Girondan témoignent d'une stabilité pluriséculaire de la rivière dans son lit actuel. En dépit de cela la ripisylve ne se reconstitue pas, la rive droite du lit majeur paraissant, au moins partiellement, être utilisée comme pâture. La surreprésentation de pollens de chanvre (autour de $30 \%$ ) signale aussi, comme pour l'époque augustéenne, une activité de rouissage. Pour des raisons déjà évoquées, la culture de cette espèce tend alors à remplacer la céréaliculture dans le lit majeur. Sur les berges elles-mêmes, aucune tentative de régulation ne pallie la submersion des aménagements antérieurs. En fait, cette absence de témoins matériels d'une occupation postérieure à l'Antiquité, notamment d'ouvrages de stabilisation de berge, s'explique sans doute en partie par la diminution progressive de la contrainte hydrique plutôt que par un abandon de cette zone.

\section{LE MARAIS}

Il est difficile d'attester une utilisation du marais au haut Moyen Âge, les canaux de drainage n'étant plus entretenus après leur colmatage au cours de la phase précédente.

Le diagramme palynologique réalisé dans la mare, en révélant un accroissement brutal des pollens d'arbres, suggère à première vue un abandon du marais et une reconquête de la forêt (fig. 20). Le pin (espèce héliophile, recolonisatrice) ainsi que le chêne se développent, et le sapin réapparaît. Au contraire, le recul du milieu herbacé se caractérise par la diminution des Graminées et des Cypéracées. Seules progressent les fougères, inféodées au milieu forestier. L'analyse des microfossiles non polliniques, peut-être plus révélateurs de situations ponctuelles, incite pourtant à moduler cette hypothèse. Si le développement d'un milieu mésotrophique pouvant être relié à un abandon des pratiques culturales est signé par la présence de certaines cyanobactéries (disparition des Spyrogira et Zygnema et remontée des Rivularia), d'autres taxons (Aphanizomenon, Anabaena et surtout Gloetrichia) indiquent la persistance d'apports organiques (déjections animales) résultant d'une activité pastorale. Cette apparente contradiction peut toutefois éventuellement s'expliquer par la pratique d'un pâturage en sous-bois ou par l'existence d'un espace végétal en mosaïque (bosquets d'arbres pollinisant beaucoup à côté de clairières riches en herbacées).

L'hypothèse d'une utilisation du marais du Grand-Plan est confirmée par la découverte d'un mur de stabilisation de la berge du Girondan au lieu-dit le Pont romain dans la partie la plus basse du marais (fig. 14). La datation radiocarbone - entre 668 et 847 -, obtenue sur un pieu de fondation, témoigne d'une tentative de protection des rives postérieure au colmatage des réseaux drainants. Plutôt qu'un marais abandonné et reconquis par la forêt, il est préférable de reconstituer un assemblage de prairies humides et de bosquets, partie prenante du domaine utile de l'exploitation et affecté à un élevage extensif.

À partir de la fin du VII ${ }^{\mathrm{e}} \mathrm{s}$. au plus tôt, les parties les plus déprimées du marais connaissent une turbification. Dans les parties plus hautes, un sol humifère brun-noir similaire, déjà rencontré sur le site et sur les berges du Girondan, se met progressivement en place. Ce retour à des conditions hydromorphes est à rattacher à l'exhaussement du plancher du lit du Girondan à la fin de l'Antiquité et au début du haut Moyen Âge.

Après un hiatus de plusieurs siècles, on assiste à de nouvelles tentatives d'aménagement du marais. Les dates 
qui permettront de les raccorder aux épisodes d'occupation ultérieurs reconnus sur le site ne sont pas encore disponibles.

\section{SYNTHÈSE}

Si la villa qui se développe entre le $\mathrm{I}^{\mathrm{er}}$ et le $\mathrm{V}^{\mathrm{e}} \mathrm{s}$. sur toute la clairière du Vernai représente l'épisode le plus marquant dans l'occupation de ce site, celui-ci ne peut se comprendre que par une prise en compte de son évolution dans la diachronie. Il est actuellement difficile de remonter aux débuts d'une fréquentation qui se perd dans la Protohistoire ancienne ou même peut-être dans la Préhistoire. D'ores et déjà, malgré la faiblesse des surfaces fouillées, il faut insister sur l'importance de l'épisode allobroge. Loin d'être un établissement réduit et éphémère, la ferme laténienne met en valeur, vraisemblablement dès le $\mathrm{II}^{\mathrm{e}} \mathrm{s}$. voire le $\mathrm{III}^{\mathrm{e}} \mathrm{s}$., non seulement les terrains limoneux de la levée alluviale mais aussi une partie du marais au prix d'un effort de drainage ou d'irrigation. Le choix de la butte du Vernai pour y implanter un site doit s'analyser selon un double niveau de lecture. À l'échelle régionale, l'Isle-Crémieu et ses piémonts verrouillent la vallée du Rhône et les points de passage empruntés par les voies de communication à grande distance se dirigeant vers les cols du nord des Alpes. Le groupe humain contrôlant l'oppidum de Larina, qui occupe à l'évidence la place centrale dans ce dispositif, ne pouvait se suffire des ressources agronomiques très limitées du plateau. Il était donc logique que des exploitations agricoles se soient implantées dans la plaine. On peut penser que la densité exceptionnelle de fermes laténiennes recensées dans ce petit secteur au regard du vide constaté, par exemple dans le Velin, est significative d'une recherche de terres à proximité immédiate de l'oppidum. À une échelle plus locale, la carte archéologique du piémont ouest de l'Isle-Crémieu montre clairement l'attraction qu'exercent les dépressions humides pendant la Protohistoire récente (fig. 3). La mise en place de ce réseau de points de peuplements diffus au pied d'un oppidum peut être comparée à des situations semblables rencontrées dans le domaine méditerranéen, par exemple au pied des Alpilles (Mauné, 2000). La terrasse fersiallitique est négligée par les habitats et consacrée au domaine funéraire. L'économie de la ferme du Vernai, basée sur la céréaliculture (orge?), l'élevage bovin, utilisateur de prairies et consommateur de fourrage et, éventuellement, la culture du chanvre explique cette attirance pour les sols lourds et humides. Sous réserve d'un drainage et peut-être d'une irrigation, ces cuvettes d'une taille relativement réduite se trouvent être, avec les sommets de moraines plaqués de loss, parmi les rares terrains susceptibles de porter de substantielles récoltes de céréales. Ces types de sols qui n'occupent qu'une fraction limitée de la vallée sont malgré tout suffisamment étendus pour fournir la cinquantaine d'hectares composant l'espace agricole utile nécessaire à une ferme, selon le calcul qui a pu être proposé pour la Gaule non méditerranéenne (Malrain et al., 2002). En s'installant sur le sommet de la levée alluviale, les habitants de cette ferme se protégeaient de la majeure partie des accidents hydrologiques tout en disposant d'un espace que ne leur aurait pas fourni, par exemple, le sommet de la butte morainique. Bien qu'il contrôlât la dépression du Grand-Plan, une des plus importantes du secteur, rien n'indique que l'habitat du Vernai ait acquis dès cette époque une importance particulière. Il s'inscrit au contraire dans une série comprenant au minimum six habitats similaires dans cette portion de vallée. Son évolution ultérieure sera, en revanche, exceptionnelle sinon par la durée de son occupation, du moins par l'ampleur que prendra le gisement.

$\mathrm{Au}$ contraire de ce qui est le plus couramment observé dans le nord de la région Rhône-Alpes (De Klijn et al., 1996), la plupart des sites fondés à cette période dans et au pied de l'Isle-Crémieu perdureront et seront à l'origine des plus grosses villae du secteur. Ainsi, même si la villa initiale connaît un important développement à l'époque augustéenne et a pu apparaître, il y a peu, comme un établissement de conquête de terroir (Berger et al., 2003), elle est en réalité le légataire d'un domaine séculaire largement organisé autour de l'exploitation des milieux humides. L'implantation à proximité immédiate d'une zone insalubre, en contradiction avec les prescriptions des agronomes, s'explique ainsi par un choix datant de l'époque protohistorique et non pas par une soudaine croissance de la demande des villes de Vienne ou Lyon ou par une pénurie locale de terres.

Malgré la rupture que représente l'introduction d'un modèle architectural méditerranéen, cette continuité entre la ferme protohistorique et la villa d'époque augustéenne est confirmée par la permanence, jusqu'à la fin du $\mathrm{I}^{\mathrm{er}}$ s. apr. J.-C., d'un vaisselier traduisant des habitudes culinaires de tradition protohistorique et, semble-t-il, par une certaine identité des productions agricoles. Ainsi le cheptel de la villa initiale, où le porc est surreprésenté, conservet-il une grande parenté avec celui des établissements protohistoriques de la région. La mutation que connaît le site révèle l'intégration d'un aristocrate allobroge dans 
la formation sociale gallo-romaine plutôt que l'apparition d'une nouvelle classe dirigeante d'origine romaine. Cette romanisation peut apparaître très précoce par rapport à la situation la plus communément répandue (Ferdière, 1988) mais semble en fait assez courante dans l'aristocratie allobroge (Jospin, 2002). La villa de la Grange à Genève fournit ainsi un exemple similaire de grand établissement allobroge remodelé à la romaine dès la fin du $\mathrm{I}^{\mathrm{er}} \mathrm{s}$. av. J.-C. (Haldimann et al., 2001). Dans l'Isle-Crémieu, malgré les difficultés pour discerner un site de La Tène finale sous une villa précoce, les prospections ont montré que les habitats accédant au statut de villae ont presque toujours une origine protohistorique.

En dépit de cette continuité d'occupation, la seconde moitié du I ${ }^{\text {er }}$ s. av. J.-C. est une période de mutation, voire de métamorphose du site dont l'importance augmente brutalement. Le caractère le plus spectaculaire de cette nouvelle dimension est certainement l'ampleur des travaux induits par la gestion de l'humidité. Elle prend dans la villa une importance très variable selon les secteurs. Curieusement, la première tentative de protection de l'habitat contre l'eau reste assez rudimentaire. Il ne semble pas exister de réseau d'évacuation hiérarchisé. Il n'est, de plus, pas certain que les techniques de construction aient été sciemment destinées à contrecarrer les effets de l'humidité. La principale parade aux dégâts de l'eau repose en fait dans l'organisation du bâti et des activités. L'habitat est construit hors de portée des crues, sur un promontoire. Les parties basses de la clairière, et notamment les berges de la rivière, sont occupées par des activités ne nécessitant que de faibles infrastructures. La mise en place de drains très divers et la tentative de colmatage des chenaux du Girondan les plus proches de l'habitat montrent que la lutte contre le risque fluvial et la remontée de la nappe aquifère sur le site fut une ouvre de longue haleine.

Il ne faut toutefois pas minimiser la dimension de l'effort d'aménagement consenti au milieu du I ${ }^{\mathrm{er}} \mathrm{s}$. av. J.-C. Les marais du Grand-Plan et de la Besseye font alors l'objet d'une opération ambitieuse d'assèchement et de mise en culture. Le réseau drainant préexistant, dont l'ampleur reste à évaluer, est totalement repris et le cours de la rivière est rectifié en bordure du site mais aussi, vraisemblablement, dans sa traversée du marais. Ce nouveau système ambivalent permet le développement de cultures céréalières discernables dans les diagrammes polliniques. Ceux-ci montrent aussi un net recul des espaces boisés (notamment des ripisylves et de la chênaie). Cette phase correspond donc à une entreprise cohérente et globale de mise en valeur des cuvettes humides qui paraissent être alors le cœur de l'infield du domaine. Il reste pourtant à évaluer le potentiel agricole réel de ce terroir qui reste d'une dimension moyenne. On peut douter que l'exploitation du ou des marais ait permis une accumulation de richesses suffisante pour bâtir la nouvelle résidence qui se met en place au début du $\mathrm{I}^{\mathrm{er}} \mathrm{s}$. Le propriétaire investit vraisemblablement dans cette reconstruction des revenus issus d'autres sources que l'exploitation du domaine. Il faut signaler que la taille énorme donnée immédiatement à cet établissement ne permet de le comparer à aucune autre villa de la région.

La rupture que marque la transformation d'une ferme en un établissement résidentiel ne paraît pas devoir être mise en parallèle avec une mutation économique. Rien ne permet de distinguer des évolutions notables dans le système de mise en valeur du marais - dont les fossés bénéficient de plusieurs phases de curage - ou dans les productions agricoles jusqu'à la seconde moitié du I ${ }^{\mathrm{er}} \mathrm{s}$. La mutation économique ne s'opère qu'à la fin de l'époque flavienne. Les deux premiers siècles de notre ère vont d'ailleurs connaître une dissociation entre les évolutions de l'agrosystème et les transformations architecturales de la villa.

La reconstruction ex nihilo de l'époque tibérienne se caractérise notamment par une séparation nettement marquée des espaces économiques et de l'habitat. Un souci particulier de traitement de l'humidité dans la partie résidentielle est rendu sensible par la mise en place de techniques architecturales adaptées et par l'installation d'un important et complexe réseau d'assainissement. Cette volonté apparaît moins pressante hors du cœur résidentiel où, pourtant, le problème est plus crucial. Au contraire de l'époque précédente où le Girondan était repoussé le plus loin possible du site vers l'ouest, on constate que la nouvelle assiette de la villa intègre la rivière. Pourtant aucun parti esthétique ou ludique n'est tiré de ce cours d'eau qui ne servira, jusqu'à la fin de l'Antiquité, qu'au fonctionnement de la pars rustica. L'eau propre et potable paraît provenir de sources extérieures au site et acheminées par des conduites. Malgré les débordements épisodiques que connaît le Girondan, il faut constater l'absence de tout dispositif protecteur contre l'eau alors que des bâtiments sont édifiés à sa proximité immédiate. Les fluctuations du régime de la rivière ne sont pas contrôlées pendant le HautEmpire. Les berges du Girondan et sa plaine d'inondation, qui forment un large ruban séparant la villa en deux parties à peu près égales, paraissent être peu densément occupées et consacrées à des activités ne nécessitant que des infrastructures très légères (rouissage du chanvre, battage des 
céréales, pacage...). Dans l'état actuel des connaissances, la pars rustica ne se présente pas comme une vaste cour unique à l'intérieur de laquelle seraient alignées des constructions, mais comme l'assemblage de trois ou quatre bandes dissemblables. À l'intérieur de l'enceinte se côtoient des parties cultivées et d'autres bâties. Un certain flou subsiste sur les modalités d'organisation et les fonctions imparties à la zone occidentale encore très mal connue.

Le fundus, en particulier ses zones humides, fait l'objet d'une importante restructuration à la suite de la crise hydraulique de seconde moitié du $\mathrm{I}^{\mathrm{er}} \mathrm{s}$. Seule une partie du réseau drainant mis en place au $\mathrm{I}^{\mathrm{er}} \mathrm{s}$. av. J.-C. est remis en état après son colmatage. Une fraction importante du marais est alors reconvertie en prés ou en garennes. Parallèlement, de nouvelles productions sont développées dans d'autres parties du domaine, qui est réorganisé. La prospection systématique n'a fourni aucun site pouvant être qualifié de villa dans un rayon de $3 \mathrm{~km}$ autour du Vernai (fig. 12). L'espace est, en revanche, parsemé d'annexes rurales, essentiellement localisées sur la basse terrasse du Rhône et sur les buttes, de fabriques de tuiles ou de poteries communes implantées le long des voies, ainsi que de plusieurs fermes qui exploitent les terres humides, notamment autour du marais de la Besseye. Si les activités artisanales semblent éphémères et secondaires, à l'exception des productions de textile couramment attestées sur le site, l'arboriculture, notamment la viticulture, paraît acquérir une importance particulière. La céréaliculture ne disparaît pas et il n'est même pas certain que son importance régresse, mais elle devient moins facilement détectable en se déplaçant sur les terrains secs. La polyculture qui s'affirme au $\mathrm{II}^{\mathrm{e}} \mathrm{s}$. amoindrit l'importance du marais dans le domaine, sans que l'on puisse dire si cette évolution est volontaire ou simplement consécutive à l'accident hydrologique de la fin du siècle précédent. Le Grand-Plan n'est plus le centre du terroir de la villa, alors que l'amélioration des conditions hydrologiques par rapport au $\mathrm{I}^{\mathrm{er}} \mathrm{s}$. rend son exploitation plus aisée. Son affectation tournée vers l'élevage ne sera pas remise en cause jusqu'à la destruction de la villa. Les fossés restaurés au début du $\mathrm{II}^{\mathrm{e}}$ s. sont, pour la plupart, entretenus jusqu'au $\mathrm{V}^{\mathrm{e}} \mathrm{s}$.

Le site connaît au $\mathrm{III}^{\mathrm{e}} \mathrm{s}$. une crise maîtrisée. Pour la première fois, une dégradation des conditions naturelles a des conséquences directes sur la villa. Aux inondations et à la remontée de la nappe répond une restructuration importante de la partie résidentielle. Les parties les plus humides de l'habitat sont abandonnées, alors que les zones économiques sont drainées (plaine d'inondation du Girondan) et que l'on tente alors de canaliser la rivière et d'en stabiliser les berges. Des bâtiments agricoles sont reconstruits et l'on constate, de manière générale, assez peu de transformations entre le système productif $\mathrm{au} \mathrm{II}^{\mathrm{e}} \mathrm{s}$. et celui mis en place après le $\mathrm{III}^{\mathrm{e}} \mathrm{s}$. Certains indices comme la multiplication des zones thermales et, plus généralement, des installations liées à l'hygiène et à l'agrément mais aussi la chasse au cerf mettent en évidence une présence aristocratique permanente jusqu'à un moment avancé $d u \mathrm{~V}^{\mathrm{e}} \mathrm{s}$. La villa du BasEmpire présente, du fait de multiples reconstructions, un aspect assez différent de celle du Haut-Empire. Le contrôle des terrains humides semble y être plus efficace, au moins sur le site et à proximité de celui-ci.

La villa du Vernai apparaît comme le représentant d'une série de grands établissements ruraux installés au bord de vallées ou de petites dépressions humides. Si l'Isle-Crémieu est quadrillée par un réseau particulièrement dense, il semble aujourd'hui que le choix de ce type d'implantation soit assez courant dans l'arrière-pays viennois. Cette prédilection paraît d'ailleurs spécifique aux campagnes allobroges et ne semble pas se retrouver sur la rive droite du Rhône.

Sa disparition à la fin du $\mathrm{V}^{\mathrm{e}} \mathrm{s}$. ou au début du $\mathrm{VI}^{\mathrm{e}} \mathrm{s}$. ne se traduit pas par un abandon du finage ni du site, même si le colmatage définitif des canaux de drainages du marais et de la plaine du Girondan marque la fin de toute tentative de gestion globale du site et de son environnement. Une nouvelle exploitation, peut-être mise en place au VII ${ }^{\mathrm{e}}$ s., reproduit à plus petite échelle la structure domaniale antique. Ses possibilités d'intervention sur son environnement semblent, il est vrai, plus limitées que celles de la villa mais, par de nombreux traits de son économie, cette exploitation paraît prolonger des tendances déjà sensibles à la fin de l'Antiquité. Si les champs disparaissent définitivement dans le marais au profit de prairies humides propices à l'élevage ou de pinèdes, il semble que la plaine du Girondan soit le lieu d'une production de chanvre et, peut-être, de céréales. Les données sont toutefois actuellement insuffisantes pour en évaluer l'importance.

Les bâtisseurs de cette résidence de plusieurs ares, non contents de réemployer des matériaux de la villa, semblent encore influencés par certains partis pris architecturaux hérités de la période gallo-romaine. Il semble pourtant exister actuellement un hiatus de près d'un siècle entre les derniers niveaux de la villa et l'établissement qui lui succède. Une chronologie plus fine de l'abandon de la villa passerait toutefois par la fouille des $500 \mathrm{~m}^{2}$ de bâtiments antiques sous-jacents à la résidence du haut Moyen Âge. 
Le mauvais état de conservation de la stratification de cette occupation interdit d'en évaluer la durée et d'étudier l'évolution du site.

Pendant la seconde moitié du haut Moyen Âge un paléosol brun-noir se développe et recouvre en grande partie le marais, les berges du Girondan, mais aussi les vestiges de l'habitat. Il indique clairement la stabilité pluriséculaire des paysages environnants, favorisée par la rétraction des champs cultivés. Cet immobilisme sédimentaire ne permet plus de suivre et de mettre en séquence les transformations économiques et environnementales médiévales.

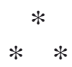

Le site du Vernai et les zones humides qui l'environnent offrent des conditions particulièrement favorables pour mener, conjointement à une fouille des espaces bâtis, un faisceau d'études à la charnière des sciences de l'environnement et de l'archéologie. L'opportunité s'est présentée de pouvoir coupler, par une étude bénéficiant d'une programmation pluriannuelle, l'examen d'une unité environnementale vaste avec la fouille du site dont elle dépend.

L'approche interdisciplinaire développée dans les zones humides autour de cette villa ne s'est pas limitée à une étude naturaliste, mais a notamment permis d'appréhender certains aspects de l'évolution économique de l'exploitation. Elle a ainsi, dans une certaine mesure, pu pallier notamment l'absence de fouille de la plus grande partie des installations agricoles. En retour, les résultats obtenus par les différentes disciplines mises en ouvre ont pu être mises en perspective et plus facilement harmonisées grâce à l'existence d'une recherche sur l'habitat, qui a fourni une problématique historique et un phasage de référence. Dans le marais, certains épisodes, dont les témoins sont peu denses ou ténus, ont été pris en considération plus aisément grâce à la mise en évidence de l'habitat contemporain sur le site. Il est peu probable qu'une attention ait été portée aux échantillons palynologiques du haut Moyen Âge si l'habitat correspondant n'avait pas été fouillé.

Il faut répéter que les mutations d'un tel site ne peuvent être appréhendées uniquement au regard des évolutions climatiques, hydro-sédimentaires ou végétales. Ainsi, des événements pourtant particulièrement importants pour l'agrosystème comme, par exemple, l'épisode de crues de la fin du $\mathrm{I}^{\mathrm{er}}$ s. apr. J.-C. associé à une remontée de l'aquifère, ne paraissent pas avoir affecté l'habitat. Les occupants du site disposaient, en tout cas pendant la période gallo-romaine, des moyens leur permettant, sinon de s'affranchir des conséquences des crises et mutations hydro-sédimentaires, du moins de les transcender. A contrario, certaines évolutions majeures de la villa, comme celle connue au début du $\mathrm{I}^{\mathrm{er}} \mathrm{s}$. ne se traduisent pas par des transformations du milieu ou du système de production.

Le bilan qui peut être aujourd'hui tiré de ce travail en cours reste provisoire et partiel. Une importante proportion du marais ainsi que la partie nord du site sont restés en dehors de l'étude. Les sols perméables et arides qui représentent pourtant l'essentiel des surfaces disponibles autour du site sont peu propices à ce genre d'études et mal connus. Leur utilité pour la dynamique du domaine est donc vraisemblablement sous-estimée. Sur le site, la cohérence des connaissances est variable selon les époques, et le développement des fouilles tend à fragmenter le processus des évolutions architecturales et économiques. Ainsi l'occupation protohistorique reste largement méconnue, notamment pour ses épisodes les plus anciens. La prescription d'une approche à la fois diachronique et exhaustive, entamée en 1999 par la fouille des niveaux médiévaux, a entraîné le décapage d'une vaste surface de vestiges non étudiés. Il existe un hiatus entre l'occupation mérovingienne et la villa gallo-romaine que seule la fouille des vestiges affleurants permettra de combler. La villa du Bas-Empire, particulièrement imposante et aux stratigraphies bien conservées, reste mal connue.

L'amélioration de nos connaissances sur l'usage du marais du Grand-Plan et des marges du site passe par un changement méthodologique. Jusqu'à présent l'approche stratigraphique, qui a permis de dégager rapidement un phasage fiable, a été privilégiée. Il conviendrait, dans le futur, d'entreprendre des décapages qui, seuls, permettront d'appréhender les aménagements ponctuels ou limités et, peut-être, de mieux comprendre l'organisation et l'utilisation des espaces à l'intérieur du domaine. 


\section{BIBLIOGRAPHIE}

Balmelle C.

2001: Les Demeures aristocratiques d'Aquitaine: société et culture de l'Antiquité tardive dans le sud-ouest de la Gaule, Bordeaux, Fédération Aquitania (Suppl. à Aquitania 10), $497 \mathrm{p}$.

\section{BARAT Y.}

1999 : «La villa de Richebourg (Yvelines)», Revue archéologique du Centre, 38, p. 117-167.

Barberan S., FAbre L., MaUfras O., Pétitot H., Pomarèdes H., Sauvage L., THERNOT R.

2002: «Les villae de la Ramière à Roquemaure, Gard ». in COLlECTIF, Archéologie du TGV Méditerranée. Fiches de synthèse, t. 3, Lattes, Association pour la recherche archéologique en Languedoc oriental (coll. Monographie d'archéologie méditerranéenne, 10), p. 889-919.

\section{BERGER J.-F.}

2001 : «L'étude géoarchéologique et paléoécologique de fossés bordiers d'époque historique: démarche, méthodes et apports à l'histoire agraire rhodanienne ", in GUILAINe J. (DIR.), Dossier hors série sur l'archéologie agraire, Paris, Études rurales, p. 59-90.

\section{BERGER J.F., ROYET R., ARGANT J., FOREST V.}

2003: «Une villa gallo-romaine en milieu humide. Le Vernai à Saint-Romain-deJalionas (Isère) ", in FAVORY F. (DIR.), Actualités de la recherche en histoire et archéologie agraires, Actes du $5^{e}$ colloque de l'association AGER, Besançon, 19-20 sept. 2000, Besançon (coll. Annales littéraires de l'Université de Besançon, 764), p. 157173

\section{BERNIGAUD N.}

2002: Evolution des anthroposystèmes des marais à l'ouest de l'Isle-Crémieu (Isère), de la fin de la protohistoire à l'époque moderne, mémoire de DEA, Université de Paris 1La Sorbonne, $84 \mathrm{p}$.

\section{BRUN J.-P., BORRÉANI M.}

1998: «Deux moulins hydrauliques du Haut-Empire romain en Narbonnaise: villae des Mesclans à La Crau et de Saint Pierre/Les Laurons aux Arcs (Var)", Gallia, 55, p. 279-327.

\section{Buisson A., Pelletier A.}

1983 : «Les voies romaines autour de Lyon. Essai de synthèse ", in CHEvalLIER R. (DIR.), Les voies anciennes en Gaule et dans le monde romain occidental, Actes du colloque de Tours, 5-6 juin 1982 (coll. Caesarodunum, XVIII), p. 157-166.

\section{DE Klijn H., MOtTe S., Vicherd G.}

1996 : «Éléments sur la romanisation des campagnes en Nord-Rhône-Alpes ", in BAYARD D., COLlard J.-L. (DIR.), De la ferme indigène à la villa romaine, Actes $d u$ $2^{e}$ colloque de l'association AGER, 23-25 sept. 1993 (coll. no spécial à la Revue archéologique de Picardie, 11), p. 271-287.

DUFOURG A.

1980 : «Le site de Saint-Romain-de-Jalionas dans son contexte archéologique ", Bulletin de liaison de la Direction des Antiquités historiques de Rhône-Alpes, 9, p. 10-27.

\section{ESQUIEU Y. (DIR.)}

1988 : Viviers, cité épiscopale: études archéologiques, Lyon, Direction des Antiquités historiques, DRAC Rhône-Alpes (coll. Documents d'archéologie en RhôneAlpes, 1), 127 p.

FAURE-BOUCHARLAT E. (DIR.)

2001: "Vivre à la campagne au Moyen Âge. L'habitat rural du V $\mathrm{V}^{\mathrm{e}}$ au $\mathrm{XII}^{\mathrm{e}} \mathrm{s}$. (Bresse, Lyonnais, Dauphiné) ", Lyon, Association lyonnaise pour la promotion de l'archéologie en Rhône-Alpes (coll. Documents d'archéologie en RhôneAlpes, 21), 432 p.

\section{FERDIÈre A.}

1988 : Les Campagnes en Gaule romaine -I- Les Hommes et l'environnement en Gaule rurale (52 av.J.-C./486 apr.J.-C.), Paris, Errance, $301 \mathrm{p}$.

\section{FOREST V.}

1998: «Étude archéozoologique, rue du Souvenir, Lyon, Rhône ( $\mathrm{II}^{\mathrm{e}} \mathrm{s}$. av. J.-C.) ", in DESBAT A. (DIR.), Recherches sur le début de la romanisation et sur le développement de Lyon aux premiers temps de la colonie (milieu du II $I^{e}$. av.J.-C./fin du $I^{\text {er }}$ s. av.J.-C.), Rapport multigraphié.

\section{FOREST V. $E T A L$.}

2001 : «Les restes fauniques ", in ROYET R. (DIR.), Le Vernai à Saint-Romain-de-Jalionas,
Rapport de synthèse de fouille programmée pluriannelle 1999-2001, DFS multigraphié SRA Rhône-Alpes, 6 vol.

Haldimann M.-A., ANDRÉ P., Broillet-Ramjoué E., PouX M.

2001: «Entre résidence indigène et domus gallo-romaine: le domaine antique du Parc de la Grange (GE) », Archéologie suisse, 24, p. 2-16.

JOSPIN J.-P. (COORD.)

2002 : Les Allobroges, Gaulois et Romains du Rhône aux Alpes, Catalogue d'exposition, Gollion, éd. in Folio.

\section{JOULIN L.}

1901 : Les Établissements gallo-romains de la plaine de Martres-Tolosane, Mémoires de l'Académie des inscriptions et belleslettres, XI, 1, Paris, p. 219-516, 24 pl.

\section{LACROIX F.-M.}

1883 : Histoire de Saint-Romain, Lyon, 59 p.

Malrain F., MatTerne V., Méniel P.

2002 : Les Paysans gaulois (III ${ }^{e}$ s.-52 av.J.-C.), Paris, Errance et INRAP, 236 p.

\section{MAUné S.}

2000 : «La question des premières installations rurales italiennes en Gaule Transalpine (fin du II $^{\mathrm{e}}$ s.-milieu du I $^{\text {er }}$ s. av. J.-C.) ", Gallia, 57, p. 231-260.

\section{PINET R.}

1972 : «Saint-Romain-de-Jalionas : gisement archéologique d'une tuilerie antique au lieu-dit "le Terreau" ", Évocations, 159 160 , p. 102-111.

\section{PLOTON M.}

2001 : Le Vernai à Saint-Romain-de-Jalionas : un site archéologique et son terroir. Approche par l'anthracologie des relations entre l'habitat et son environnement, mémoire de DEA, Université de Paris X-Nanterre, 51 p.

PORTE P.

2002 : Le Domaine rural de Larina de l'Antiquité tardive au haut Moyen Âge (Hièressur-Amby), thèse de Doctorat, Université d'Aix-Marseille I, 2 vol., 595 p.

\section{RÉMY B.}

2000: « À propos du Rhône comme limite de la cité de Vienne au Haut-Empire (en amont de Lyon) ", Revue archéologique de Narbonnaise, 33, p. 55-61. 
ROYET R., BERGER J.-F., BERNIGAUD N., ROYET E. avec la collab. de ARGANT J., BOUBY L., FOREST V., LOPEZ-SAEZ A.,

\section{BUI THI M.}

2004: «La gestion d'un environnement humide de La Tène au haut Moyen Âge. Le site du Vernai et le marais du Grand-Plan à Saint-Romain-de-Jalionas (Isère) ", in BURNOUf J., LEVEAU P.
(DIR.), Fleuves et marais, une histoire au croisement de la nature et de la culture, Actes du colloque d'Aix-en-Provence, 28-29 mai 2002, Paris, Ministère de l'Éducation nationale, p. 253-281.

\section{ROYET R., COQUIDÉ C.}

À paraître: "L'agglomération secondaire antique d'Optevoz (Isère) : un état des connaissances », Revue archéologique de Narbonnaise, 18 p.

\section{ROYET R., FAUCHER F.}

2002: «La villa de Sainte-Colombe et le soi-disant vicus de Travers à La Balmeles-Grottes (Isère) : réinterprétation des données archéologiques ", Revue archéologique de Narbonnaise, 35, p. 259-271. 